Overview of JET results

This content has been downloaded from IOPscience. Please scroll down to see the full text. 2011 Nucl. Fusion 51094008

(http://iopscience.iop.org/0029-5515/51/9/094008)

View the table of contents for this issue, or go to the journal homepage for more

Download details:

IP Address: 193.136.33.215

This content was downloaded on 17/07/2015 at 13:22

Please note that terms and conditions apply. 


\title{
Overview of JET results
}

\author{
F. Romanelli, M. Laxåback ${ }^{\mathrm{a}}$ on behalf of the JET EFDA \\ Contributors $^{\mathrm{b}}$
}

JET-EFDA, Culham Science Centre, Abingdon, OX14 3DB, UK

E-mail: martin.laxaback@jet.efda.org

Received 14 February 2011, accepted for publication 18 May 2011

Published 31 August 2011

Online at stacks.iop.org/NF/51/094008

\begin{abstract}
Since the last IAEA Conference JET has been in operation for one year with a programmatic focus on the qualification of ITER operating scenarios, the consolidation of ITER design choices and preparation for plasma operation with the ITER-like wall presently being installed in JET. Good progress has been achieved, including stationary ELMy $\mathrm{H}$-mode operation at 4.5 MA. The high confinement hybrid scenario has been extended to high triangularity, lower $\rho^{*}$ and to pulse lengths comparable to the resistive time. The steady-state scenario has also been extended to lower $\rho^{*}$ and $v^{*}$ and optimized to simultaneously achieve, under stationary conditions, ITER-like values of all other relevant normalized parameters. A dedicated helium campaign has allowed key aspects of plasma control and H-mode operation for the ITER non-activated phase to be evaluated. Effective sawtooth control by fast ions has been demonstrated with ${ }^{3} \mathrm{He}$ minority ICRH, a scenario with negligible minority current drive. Edge localized mode (ELM) control studies using external $n=1$ and $n=2$ perturbation fields have found a resonance effect in ELM frequency for specific $q_{95}$ values. Complete ELM suppression has, however, not been observed, even with an edge Chirikov parameter larger than 1. Pellet ELM pacing has been demonstrated and the minimum pellet size needed to trigger an ELM has been estimated. For both natural and mitigated ELMs a broadening of the divertor ELM-wetted area with increasing ELM size has been found. In disruption studies with massive gas injection up to $50 \%$ of the thermal energy could be radiated before, and $20 \%$ during, the thermal quench. Halo currents could be reduced by $60 \%$ and, using argon/deuterium and neon/deuterium gas mixtures, runaway electron generation could be avoided. Most objectives of the ITER-like ICRH antenna have been demonstrated; matching with closely packed straps, ELM resilience, scattering matrix arc detection and operation at high power density $\left(6.2 \mathrm{MW} \mathrm{m}^{-2}\right)$ and antenna strap voltages $(42 \mathrm{kV})$. Coupling measurements are in very good agreement with TOPICA modelling.
\end{abstract}

(Some figures in this article are in colour only in the electronic version)

\section{Introduction}

Since the last IAEA Conference [1] JET has been in operation for one year with a maintained programmatic focus on the qualification of ITER operating scenarios [2], the consolidation of ITER design choices and the preparation for future plasma operation with the JET ITER-like wall (ILW) [3,4]. Machine and subsystem reliability has been very good (with neutral beam power in excess of $22 \mathrm{MW}$ being achieved in several pulses) and has allowed strong progress in the JET programme, including stationary type-I ELMy H-mode operation with plasma currents up to 4.5 MA [5] and a dedicated helium campaign to evaluate key aspects of plasma control and $\mathrm{H}$-mode operation for the ITER non-activated phase. The latest experimental campaign ended in October 2009 and JET has since been in shutdown for the JET Enhancement Programme 2 (EP2) upgrades, chiefly the installation of the ILW and the

\footnotetext{
a Also at: Association EURATOM-VR, EES, KTH, SE-10044 Stockholm, Sweden.

${ }^{\mathrm{b}}$ See the appendix.
}

upgrade to the neutral beam injection (NBI) system [6]. The ILW project sees the replacement of all carbon fibre composite (CFC) plasma facing components (PFCs) with beryllium for the first wall (solid $\mathrm{Be}$ and $8 \mu \mathrm{m}$ Be-coated Inconel) and tungsten in the divertor (10-15 $\mu \mathrm{m}$ and $20-25 \mu \mathrm{m}$ W-coated CFC tiles for the inner and outer divertor, respectively [7, 8], and bulk $\mathrm{W}$ for the horizontal tile for the outer strike point in high performance scenarios $[9,10])$. The neutral beam upgrade will bring the maximum power from 22 to $34 \mathrm{MW}$, with the maximum pulse length extended to $20 \mathrm{~s}$. Before the end of the latest experimental campaign the new enhanced radial field amplifier (ERFA) [11] (together with a few new diagnostics) has been installed and fully commissioned [12], demonstrating its capability of controlling plasma vertical position with the largest edge localized modes (ELMs) [13]. Installation tasks for the EP2 upgrades are scheduled to be completed in early 2011, with plasma operation restarting later in the year.

The rest of this paper is arranged as follows: section 2 provides an overview of recent progress in the qualification 


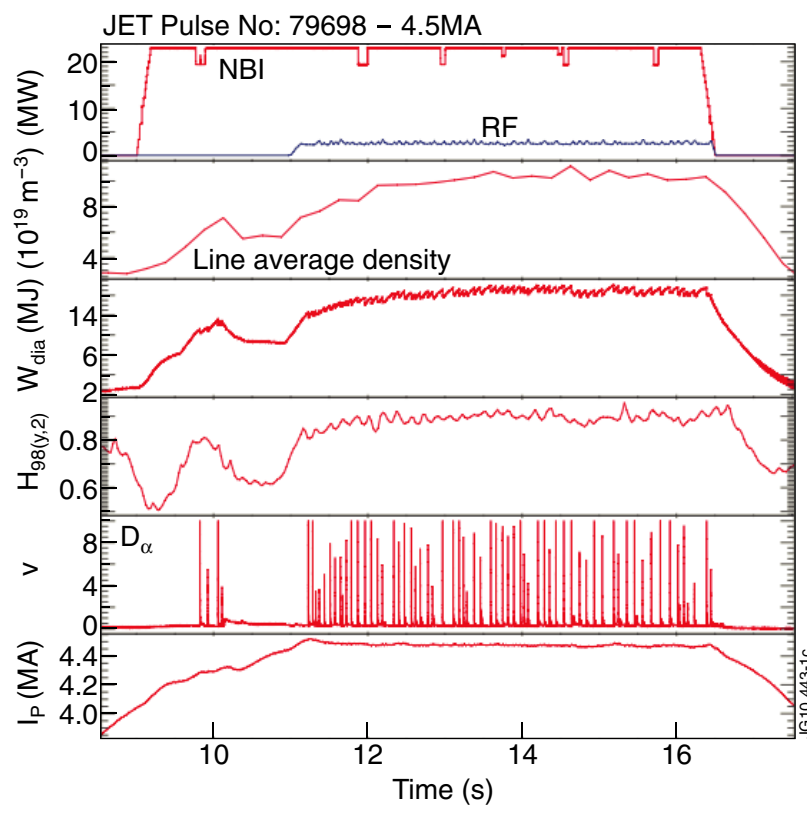

Figure 1. Stationary 4.5 MA type-I ELMy H-mode using deuterium gas fuelling to moderate the large natural ELMs.

of ITER operating scenarios, the ELMy H-mode, hybrid and advanced tokamak scenarios, as well as a summary of the qualification of helium operation for the ITER non-activated phase. Section 3 highlights some results on plasma transport and core stability issues, primarily related to momentum transport and fast particle/burning plasma physics. In section 4 results have been collected from work relating to first wall power loads, including the preparation for operating with the ILW, ELM physics and mitigation studies and disruption studies. Section 5 describes the ITER-relevant ion cyclotron resonance heating (ICRH) studies carried out in the last few years. Finally, conclusions and a brief outlook for the next few years of JET operation are covered in section 6 .

\section{Progress in the qualification of ITER operating scenarios}

\subsection{ELMy H-mode}

2.1.1. High current operation. Operation with high plasma current in JET allows access to the most ITER-relevant plasma conditions, in terms of dimensionless plasma parameters and edge pedestal characteristics, of any present day machine. Stationary ELMy H-mode operation with plasma currents up to $4.5 \mathrm{MA}$ has been achieved in low triangularity $(\delta=0.25)$ and low edge safety factor $\left(q_{95} \sim 2.65\right)$ configuration with 26.5 MW of input power, resulting in stored energies of up to 11.5 MJ, plasma densities of $55 \%$ of the Greenwald density limit $\left(n_{\mathrm{GW}}=I_{\mathrm{p}} /\left(\pi a^{2}\right)\right.$ in units of $10^{20} \mathrm{~m}^{-3}$ with $I_{\mathrm{p}}$ in MA) and normalized ion Larmor radius $\left(\rho^{*}=\left(2 m_{\mathrm{i}} T_{\mathrm{i}}\right)^{1 / 2} /(e B a)\right)$ and collisionality $\left(v^{*}=n_{\mathrm{e}} R q_{95} \ln \Lambda /\left((a / R)^{3 / 2} \times 5.73 \times\right.\right.$ $\left.10^{54} T_{\mathrm{e}}^{2}\right)$ ) down to $3 \times 10^{-3}$ and $5 \times 10^{-3}$, respectively (figure 1) [5]. $\quad \rho^{*}$ and $v^{*}$ are here calculated using the volume-averaged electron densities and temperatures and assuming $T_{\mathrm{i}}=T_{\mathrm{e}}$. Discharges with plasma currents above 3.5 MA and low deuterium fuelling displayed a nonstationary H-mode behaviour, characterized by phases of

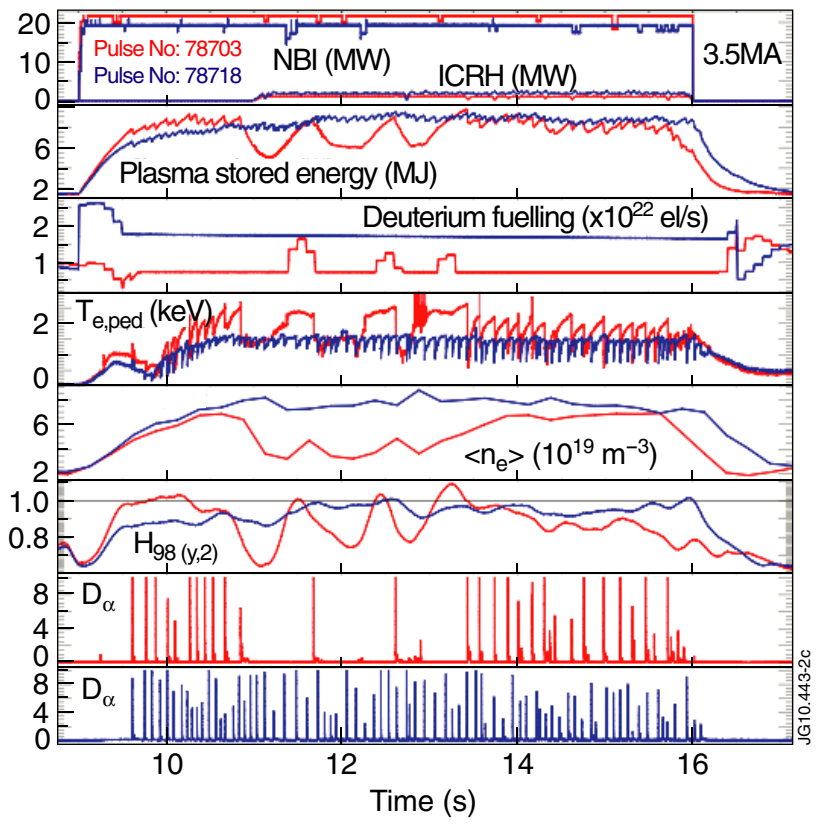

Figure 2. Unfuelled 3.5 MA discharge (red) where the impurity influx following large type-I ELMs lead to transition to periods of type-III ELMs or even L-mode, and similar fuelled 3.5 MA discharge (blue) where stationary type-I ELMs are maintained.

high and low confinement and transitions back to L-mode (figure 2). This plasma behaviour is consistent with an earlier observation that when the ELM size exceeds a threshold of $\Delta W_{\mathrm{ELM}} \sim 0.6 \mathrm{MJ}$, similar to the natural ELM size at 3.5 MA, ablation of co-deposited carbon layers in the divertor lead to impurity influx, cooling of the divertor plasma and a transition to type-III ELMs or even L-mode [14]. Reliable stationary type-I H-modes could be achieved by adding strong deuterium gas fuelling (figure 2). However, the resulting performance at the highest plasma currents falls short of the IPB98 $(y, 2)$ scaling [15] (figure 3). Analysis is still ongoing to confirm the origin of the degraded confinement, but a strong candidate is the pedestal cooling that was caused by the gas fuelling. Figure 4 shows the electron density (measured by the high resolution Thompson scattering (HRTS) diagnostic) and temperature (calculated from the average of HRTS and electron cyclotron emission measurements) profiles for a pair of 3.5 MA discharges with and without gas fuelling. While the fuelling does not significantly affect the average plasma density (constant Greenwald fraction), it does lead to higher pedestal Greenwald fractions and lower pedestal and (consistent with profile stiffness) core temperatures. Although these results refer to the specific JET divertor configuration, they indicate that ELM control may be necessary in ITER not only to limit transient heat loads but also to achieve high confinement.

2.1.2. Joint JET and DIII-D $\rho^{*}$ pedestal scaling experiments. The dependence of the edge pedestal width on the normalized ion gyroradius $\rho^{*}=\rho_{\mathrm{i}} / a$ has been explored in joint JET and DIII-D experiments. Theoretical models, based on the assumption that the pedestal width is set by the condition that the linear turbulence growth rate is equal to the $E \times B$ velocity shearing rate, lead to pedestal width $(\Delta)$ scalings with $\rho^{*}$ dependences ranging from $\Delta / a \propto \rho^{* 1 / 2}$ to $\Delta / a \propto \rho^{*}[16]$. 

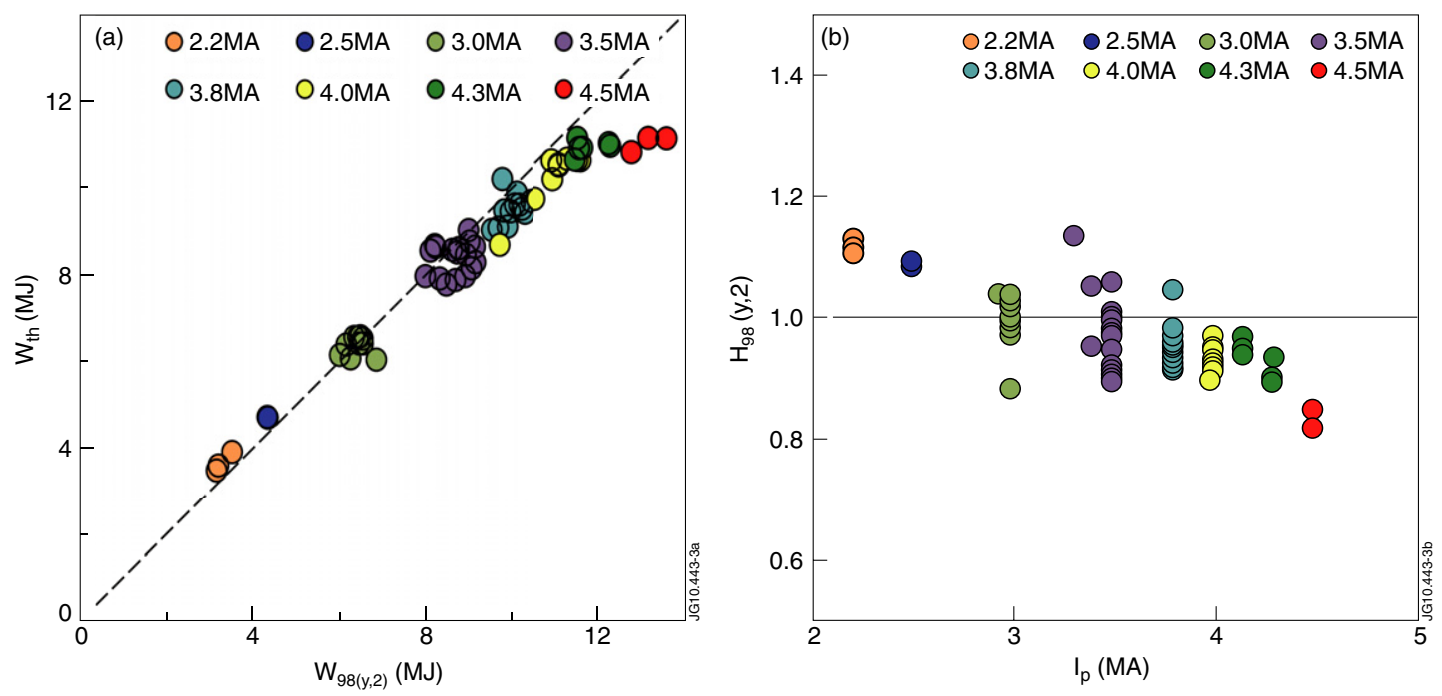

Figure 3. (a) Measured versus predicted thermal stored energy. (b) Confinement enhancement factor $H_{98(y, 2)}$ as a function of plasma current.

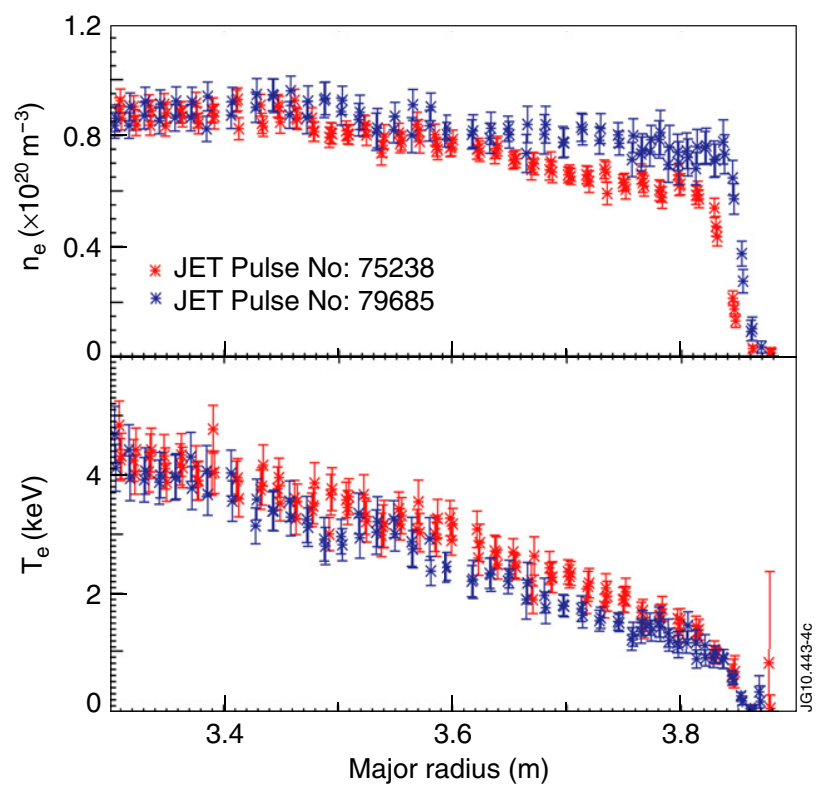

Figure 4. Electron density and temperature profiles for two 3.5 MA discharges with (blue) and without (red) gas fuelling.

For large, high-field, tokamaks like ITER this would imply narrower pedestals than in current tokamaks with implications for the achievable $\mathrm{H}$-mode confinement. Taking advantage of the difference in machine size between JET and DIII-D a factor 4 variation in $\rho^{*}$ at the top of the pedestal $\left(2.2 \times 10^{-3}\right.$ $9.3 \times 10^{-3}$ ) could be achieved around a dimensionless identity point at $I_{\mathrm{p}} / B_{\mathrm{T}}=1.0 \mathrm{MA} / 1.1 \mathrm{~T}$ for JET and $1.1 \mathrm{MA} / 2.1 \mathrm{~T}$ for DIII-D. Other dimensionless parameters $\left(v^{*}, \beta_{\mathrm{pol}}\right.$ and $q_{95}$ ) were kept constant at the top of the pedestal as the magnetic fields were varied in steps as $B_{\mathrm{T}}(\mathrm{JET})=1.1,1.8$ and $2.7 \mathrm{~T}$ and $B_{\mathrm{T}}$ (DIII-D) $=1,1.4$ and $2.1 \mathrm{~T}[16,17]$. The resulting electron temperature and density pedestal widths are plotted in figure 5. The temperature pedestal width is invariant with $\rho^{*}$ and only a weak $\rho^{*}$ dependence is found for the density pedestal width, ruling out the strong dependence of the pedestal width on $\rho^{*}$ predicted by the theoretical models referred to above and clearly an encouraging result for ITER.

2.1.3. RF-dominated H-modes. Extrapolations from present day devices to the ITER $Q_{\mathrm{DT}}=10$ baseline scenario are based predominantly on ELMy H-modes heated by positive NBI with dominant ion heating and significant toroidal momentum input. In contrast, plasma heating in ITER will be by $\alpha$-particles, negative NBI, ICRH and electron cyclotron resonance heating $(\mathrm{ECRH})$ with dominant electron heating and insignificant levels of momentum input. Exploiting the recent improvements in ELM resilience of the JET ICRH systems (see section 5) the effect of the heating mix and rotation on the core and pedestal confinement has been investigated in matched pairs of NBI-only and ICRH+NBI-heated low triangularity $(\delta=0.25) 2.5 \mathrm{MA} / 2.7 \mathrm{~T}\left(q_{95} \sim 3.6\right) \mathrm{H}$-mode plasmas with Greenwald density fractions around 60-70\% [18]. The ICRH scenario used was $42 \mathrm{MHz}$ dipole phasing $\mathrm{H}$-minority heating with $n_{\mathrm{H}} /\left(n_{\mathrm{D}}+n_{\mathrm{H}}\right)=4 \pm 0.5 \%$. $Z_{\text {eff }}$ was typically $1.7-2$. Within the limitations of available ICRH power comparisons between 100\% ICRH and 100\% NBI-heated plasmas could only be performed at relatively low power, $P_{\text {tot }} \sim 9 \mathrm{MW}$, $P_{\text {loss }} / P_{\mathrm{L}-\mathrm{H}} \sim 1$ 1.2. Comparisons between mixed ICRH+NBI and NBI-only heated plasmas were performed at progressively higher powers as the NBI fraction increased, up to $P_{\text {tot }} \sim$ $16 \mathrm{MW}, P_{\text {loss }} / P_{\mathrm{L}-\mathrm{H}} \sim 2$ for comparisons between $50: 50$ ICRH : NBI and $100 \%$ NBI-heated plasmas. $P_{\text {loss }}$ is here the lost power including radiation and $P_{\mathrm{L}-\mathrm{H}}$ is calculated using the Martin08 scaling [19]. No significant systematic differences were found between the ICRH-dominated plasmas and the matched NBI-only plasmas. The plasma confinement is found to be independent of the heating mix (figure 6) density and temperature profiles in the core are similar and $T_{\mathrm{i}} \approx T_{\mathrm{e}}$ despite the different heat, particle and torque deposition profiles (figure 7). The toroidal rotation in the ICRH-dominated plasmas was approximately ten times lower at the edge and five times lower in the core. The pedestal characteristics (pressure and width) are also independent of the heating mix and no obvious correlation is found between the ELM size and frequency and the heating mix. These results indicate 

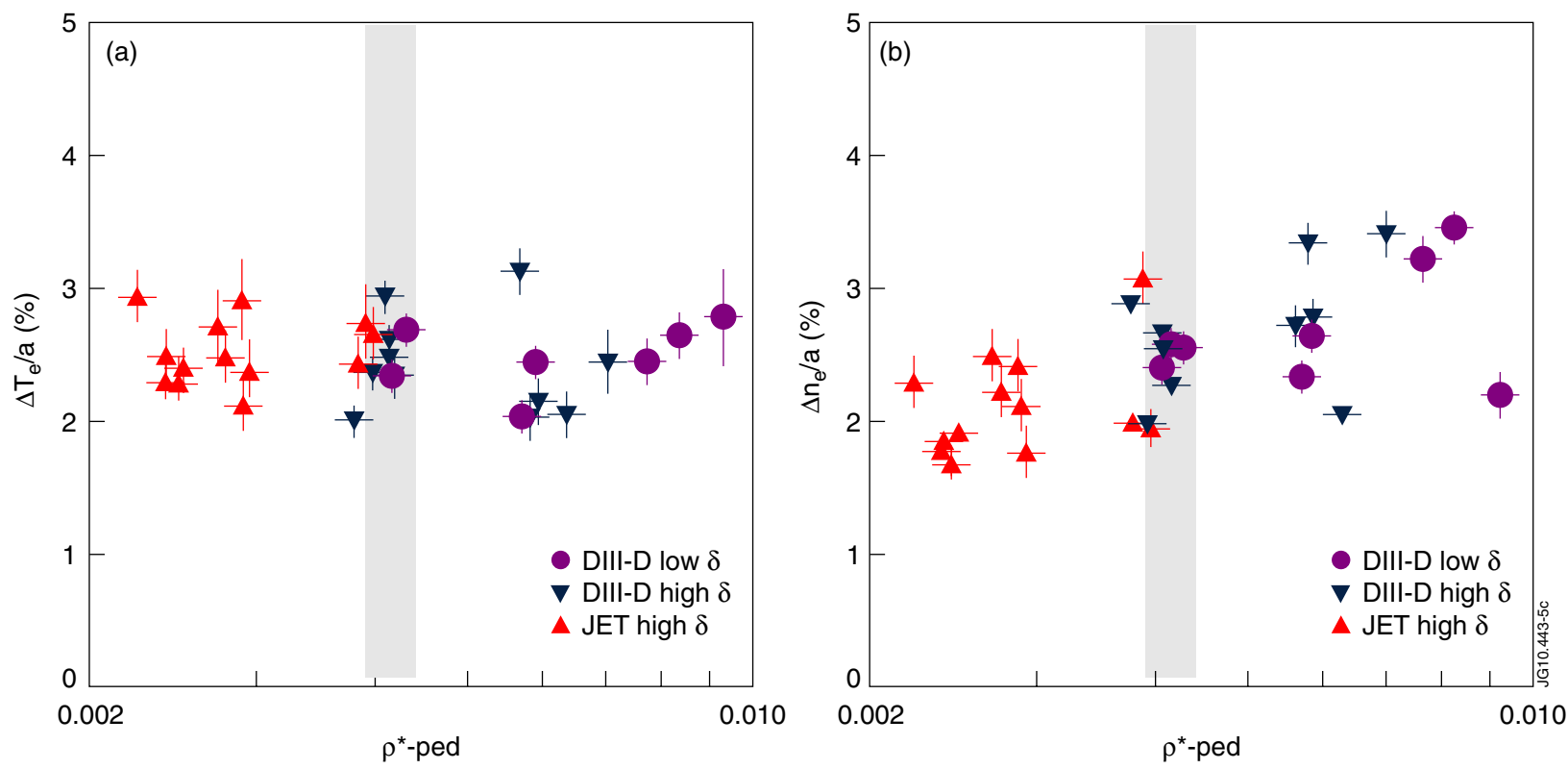

Figure 5. Pedestal structure for JET and DIII-D as a function of pedestal $\rho^{*}$. The shaded area indicates identity point. ( $a$ ) Electron temperature pedestal width $\Delta T_{\mathrm{e}}$ in $\%$ of the minor radius. (b) Electron density pedestal width $\Delta n_{\mathrm{e}}$ in $\%$ of the minor radius.

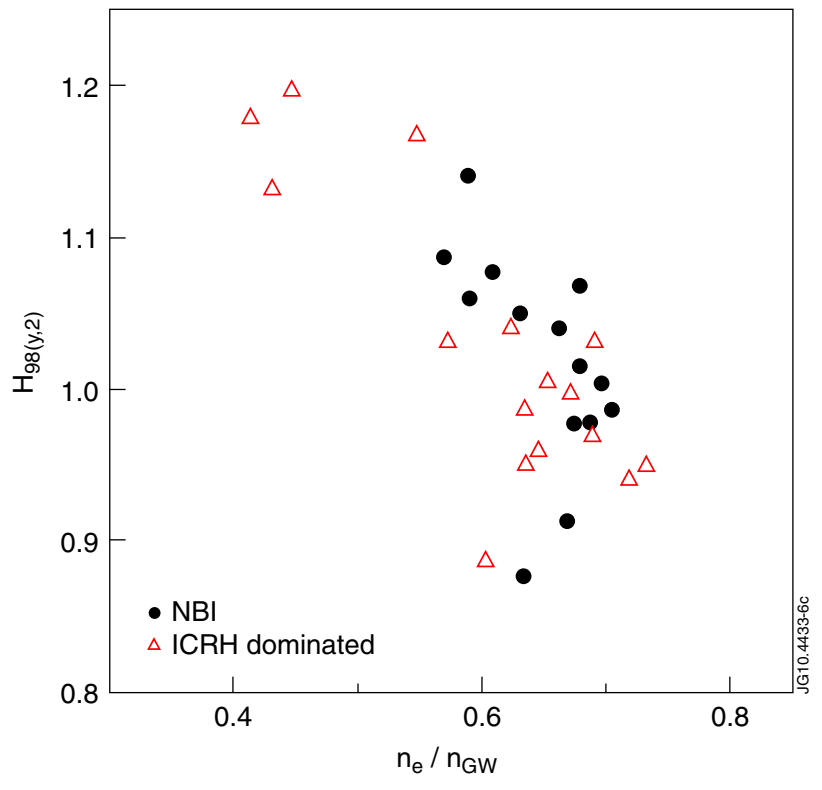

Figure 6. Confinement enhancement factor $H_{98(y, 2)}$ versus normalized density for plasmas dominated by, respectively, NBI and ICRH heating.

that $\mathrm{H}$-mode confinement scalings, despite being derived from plasmas dominated by ion heating and strong momentum input, are robust enough to describe also the behaviour of plasmas dominated by electron heating and low momentum input as will be the case in ITER.

\subsection{Hybrid scenario}

The hybrid scenario is a promising route for ITER as it might allow the achievement of $Q=10$ at lower plasma current and thereby longer pulse lengths. At the 2008 IAEA FEC it was reported that confinement enhancement up to
$40 \%$ above the $\operatorname{IPB} 98(y, 2)$ scaling was transiently achieved on JET [20]. Since 2008 the hybrid scenario has been further progressed and now routinely achieves confinement improvements over the standard H-mode. The scenario has been extended to high triangularity $(\delta \sim 0.4)$ and lower $\rho^{*}$ $\left(\sim 3.5 \times 10^{-3}\right)$ at $2.3 \mathrm{~T}$ and plasma currents up to $2 \mathrm{MA}$ with $H_{98(y, 2)}$ typically $1.3-1.4$ [21]. Pulse lengths in excess of $6 \mathrm{~s}\left(\sim \tau_{\mathrm{R}}\right.$, the resistive diffusion time $)$ have been achieved at $n_{\mathrm{e}} / n_{\mathrm{GW}} \sim 0.75$ and $\beta_{\mathrm{N}} \sim 3\left(\beta_{\mathrm{N}}=\beta_{\mathrm{T}} a B / I_{\mathrm{p}}[\%]\right)$ (figure 8). The extension of the high performance hybrid scenario to low $\rho^{*}$ breaks the negative trend in confinement with decreasing $\rho^{*}$ which was suggested in the multi-machine hybrid scenario existence diagram reported in [22] (figure 9). Recent joint DIII-D and JET dimensionless experiments show only a very weak dependence on $H_{98(y, 2)}$ with $\rho^{*}$ [23]. Current evolution reconstructions using TRANSP [24] and CRONOS [25] show the non-inductive current fraction to be around $50 \%$ (15\% beam-driven and 35\% bootstrap current), which would allow ITER to reach discharge durations of around $1000 \mathrm{~s}$.

No single origin of the improved confinement has been identified, with a current ramp-down prior to the main heating phase to tailor the target $q$-profile, careful shape control to avoid deleterious wall interactions and careful avoidance of core MHD such as neoclassical tearing modes (NTMs) all seemingly playing a role. The low magnetic shear in the plasma core together with a high toroidal rotation shear may also lead to reduced ion stiffness and contribute to the overall confinement [26]. For the same total energy content the pedestal contribution to the confinement is found to be in line with the baseline scenario, around $30-40 \%$ at high triangularity and $20-30 \%$ at low triangularity (as determined by HRTS), demonstrating that the pedestal confinement does not depend crucially on the magnetic shear. There are, however, indications that the pedestal confinement improves progressively with increasing $\beta_{\mathrm{N}}$ and the pedestal energy in 

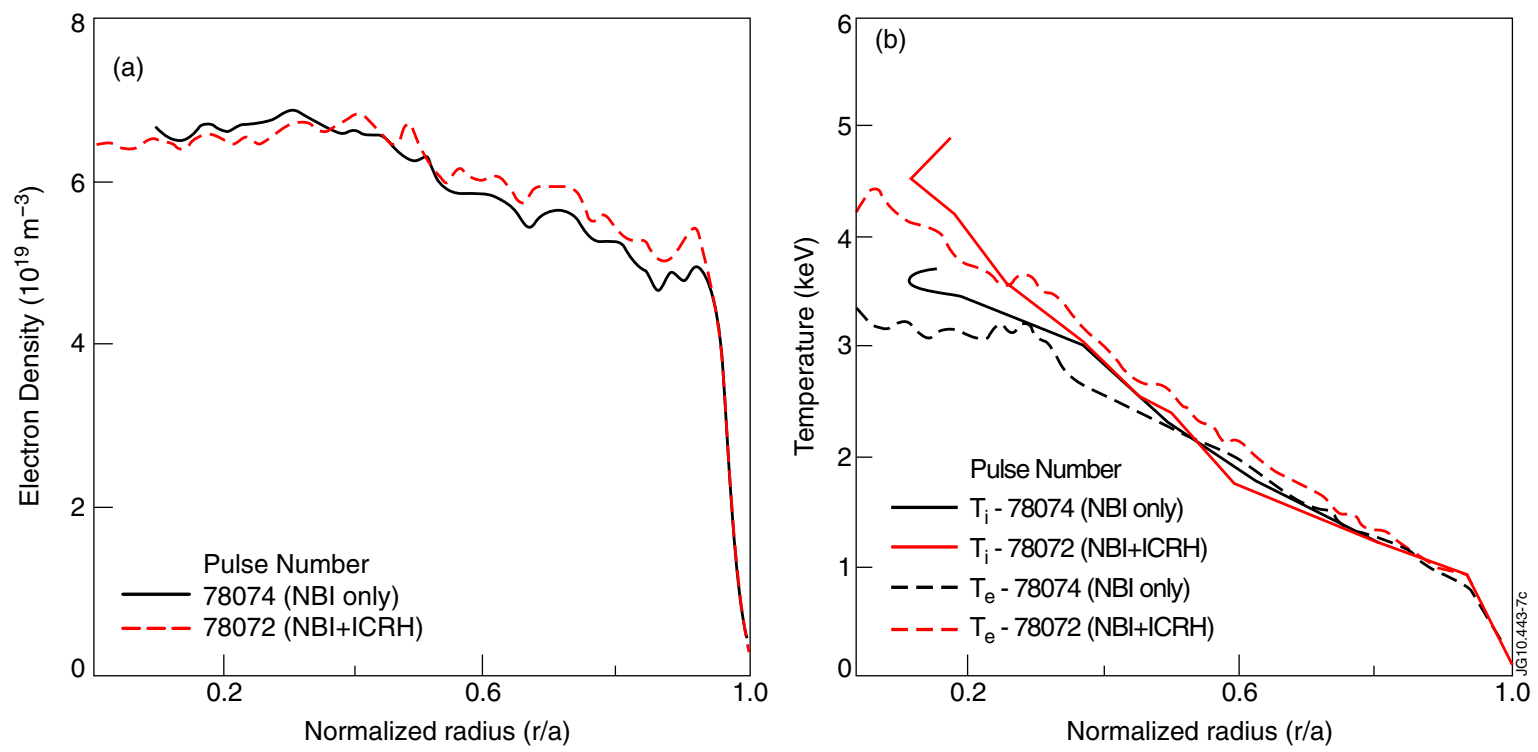

Figure 7. (a) Electron density profiles from the HRTS diagnostic. (b) Ion (from charge exchange recombination spectroscopy) and electron (from HRTS) temperature profiles for H-mode discharges heated by $100 \%$ NBI (black) and $85 \%$ ICRH (red). The total heating power was $11 \mathrm{MW}$ for both plasmas.

JET Pulse No: $77922\left(\mathrm{~B}_{\mathrm{T}}=2.3 \mathrm{~T}\right)$

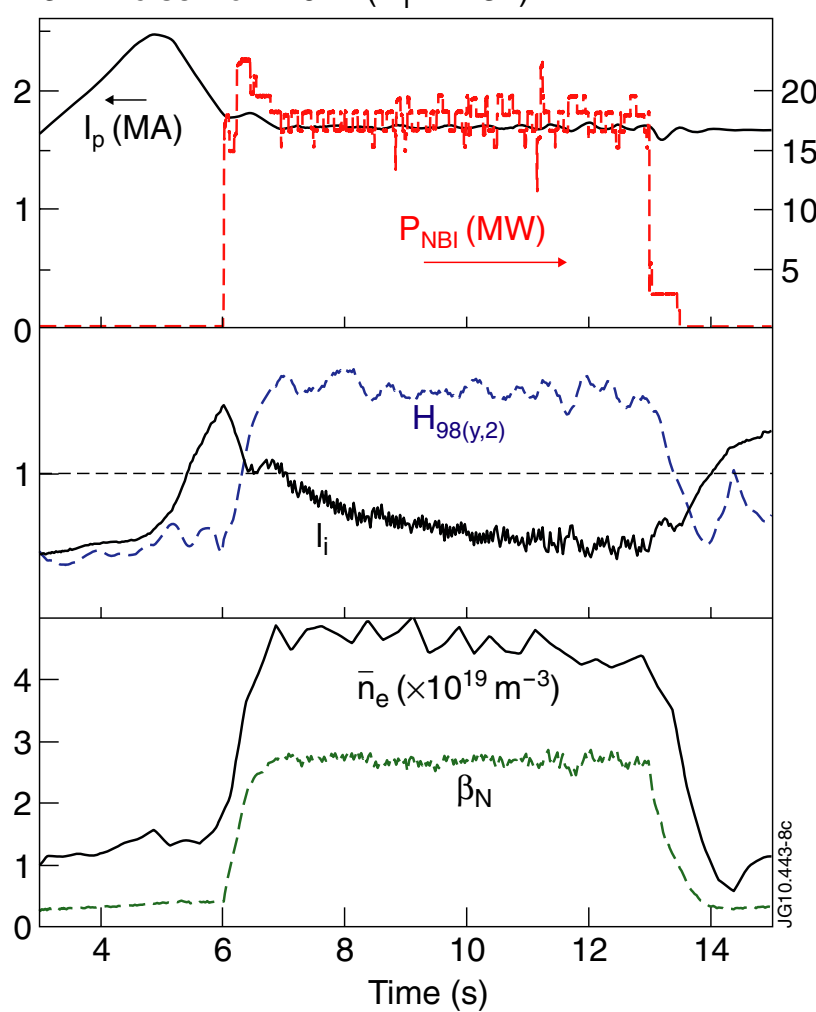

Figure 8. High triangularity hybrid discharge $(\delta \sim 0.4)$ at $75 \%$ of the Greenwald density with $H_{98(y, 2)}$ maintained at 1.3 for one resistive time. That the high confinement is maintained as the current profile evolves, as evidenced by the varying internal inductance $l_{\mathrm{i}}$, indicates that the magnetic shear is not the sole source of the improved confinement of the hybrid.

hybrid discharges with $\beta_{\mathrm{N}} \sim 3$ is higher than in similar baseline with $\beta_{\mathrm{N}} \sim 2$ for the same energy content as calculated by the IPB98 $(y, 2)$ scaling [27]. It should be noted that the IPB98 $(y, 2)$ scaling was derived from an H-mode database

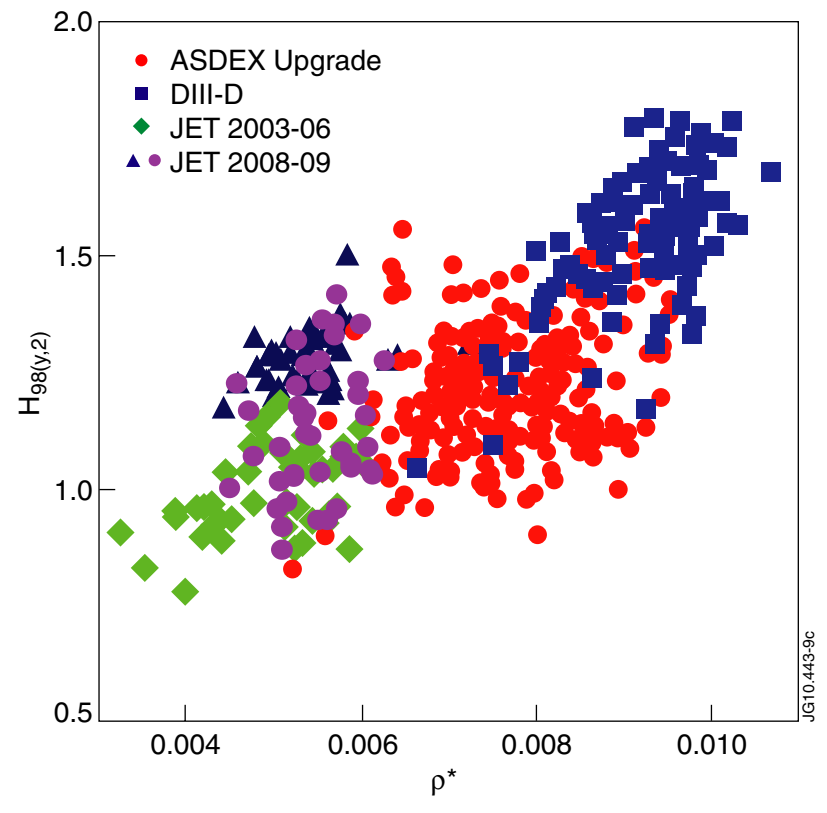

Figure 9. Confinement of recent JET low (pink) and high (dark blue) triangularity hybrid discharges compared with AUG, DIII-D and JET hybrid discharges in the ITPA database.

with $\beta_{\mathrm{N}}<2.2$ and therefore not necessarily applicable to hybrid discharges with $\beta_{\mathrm{N}}>2.2$.

\subsection{Advanced tokamak/steady-state scenario}

The performance and stability of the advanced tokamak scenario with an internal transport barrier (ITB) have been extended to $1.8 \mathrm{MA} / 2.7 \mathrm{~T}\left(q_{95} \sim 4.7\right)$, achieving dimensionless parameters approaching the ITER steady-state targets for high triangularity $(\delta \sim 0.4)$ plasmas with global $\rho^{*} / \rho_{\text {ITER }}^{*} \sim 2$ and $v^{*} / \nu_{\text {ITER }}^{*} \sim 4$ [28] (figure 10). In addition to around $22 \mathrm{MW}$ of NBI, up to $8 \mathrm{MW}$ of ICRH and typically 


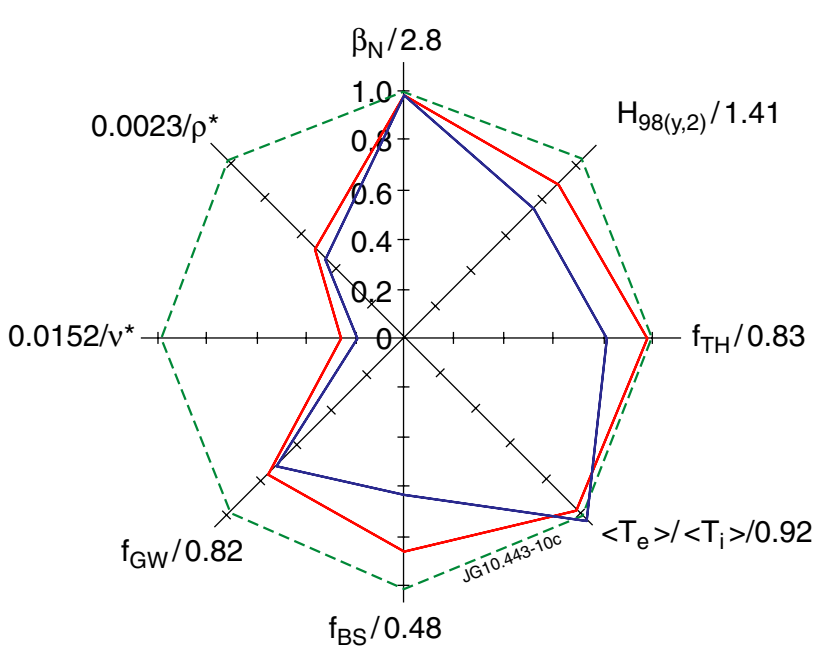

Figure 10. Dimensionless parameters for JET pulse 78052 ,

1.8 MA/2.7 T (red) normalized to ITER steady-state scenario targets compared with the best performance previously achieved, JET pulse 70069, 1.5 MA/2.3 T (blue).

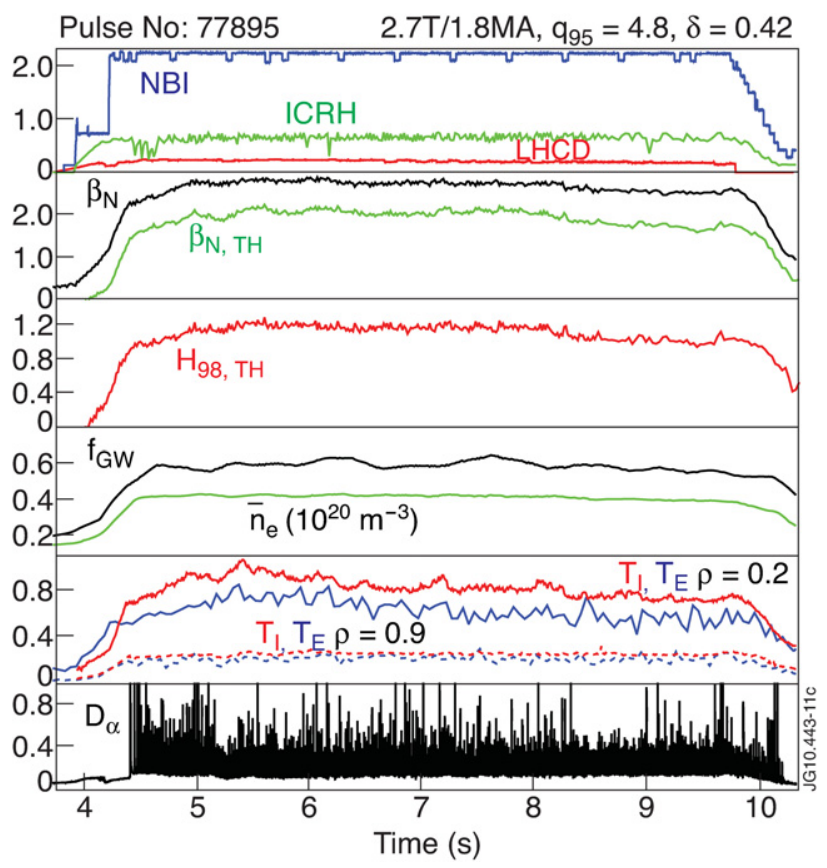

Figure 11. Evolution of JET pulse 77895 with $P_{\mathrm{NBI}}=22.5 \mathrm{MW}$, $P_{\mathrm{ICRH}}=6.6 \mathrm{MW}$ and $P_{\mathrm{LHCD}}=2.3 \mathrm{MW}$.

2.5 MW of lower hybrid current drive (LHCD) was applied to a $q_{0} \sim 2$ target chosen to optimize the bootstrap current. Relatively weak ITBs are formed (typically correlated with the $q=2$ surface rather than with negative shear) providing good plasma stability properties and allowing to simultaneously achieve $\beta_{\mathrm{N}} \geqslant 2.7, H_{98(y, 2)} \geqslant 1.2, T_{\mathrm{e}} \sim T_{\mathrm{i}}, n_{\mathrm{e}} / n_{\mathrm{GW}} \sim$ 0.65 and a large thermal energy fraction, $f_{\mathrm{TH}} \sim 0.8$, under stationary conditions $\left(\sim 10 \tau_{E}\right)$. Gas injection added to improve the ICRH and LHCD coupling lead to type-I ELMs about $40 \%$ smaller than natural ELMs and plasma conditions are clean, $Z_{\text {eff }} \leqslant 2$. Figure 11 shows the time traces for one such discharge with $P_{\mathrm{NBI}}=22.5 \mathrm{MW}, P_{\mathrm{ICRH}}=6.6 \mathrm{MW}$ and $P_{\mathrm{LHCD}}=2.3 \mathrm{MW}$. In all discharges there is, however, evidence that the $q$-profile is evolving, signifying a shortage of non-inductive current. TRANSP interpretive modelling indicates a bootstrap fraction in the range $36-45 \%$ and NBI current drive of $20 \%$ of the plasma current. CRONOS modelling predicts an additional $10 \%$ of LH-driven current around $r / a \sim 0.6$ [29]; however, experimental observations indicate that the accessibility is marginal in these plasmas and that little LH power penetrates beyond $r / a \sim 0.8$. The LHdriven current is therefore expected to be small. An additional $35 \%$ of non-inductive current drive would thus be needed to make these scenarios fully steady state. Predictive CRONOS modelling indicates that the addition of $5 \mathrm{MW}$ of ECRH in the current ramp-up phase and a total of $10 \mathrm{MW}$ of off-axis $\mathrm{ECRH} / \mathrm{ECCD}$ at $r / a \sim 0.6$ during the current flat-top phase would add 0.25 MA from ECCD and about 0.2 MA more bootstrap current due to a resulting higher plasma temperature $[29,30]$. This would bring the scenario sufficiently close to being fully non-inductive to maintain the $q$-profile needed to sustain the ITB, and hence maintain the performance, throughout the discharge duration. An ECRH/ECCD system that would be capable of delivering the required $10 \mathrm{MW}$ to the plasma has recently been proposed for JET [31]

\subsection{Qualification of helium operation for the ITER non-activated phase}

During the initial non-activated phase, ITER must operate either hydrogen $(\mathrm{H})$ or helium $\left({ }^{4} \mathrm{He}\right)$ plasmas to commission systems, develop operating scenarios for future DT operation and evaluate ELM-mitigation techniques. The high $\mathrm{L}-\mathrm{H}$ threshold power of hydrogen plasmas (around twice that of deuterium $[32,33]$ ) appears to preclude hydrogen $\mathrm{H}$-mode operation in ITER with the planned auxiliary heating power, leaving helium as the likely option. In order to qualify helium operation as a viable candidate for the ITER non-activated phase a dedicated helium campaign, with the NBI system fully converted to helium, has been carried out at JET, using the technique of argon frosting for both divertor and NBI cryopumps to ensure the best possible helium pumping.

2.4.1. ITER current ramp-up and ramp-down studies in helium. One of the first and most fundamental tasks during the ITER non-activated phase will be the commissioning of critical tokamak subsystems for plasma vertical control. JET performed ITER scenario demonstration discharges in deuterium in 2008 [34] that contributed to the modification of the ITER coil design. These discharges have now also been used as references for a new set of discharges in helium to qualify the flux consumption and heating requirements for current profile control during the current-rise, $q_{95}=3$ flat top and current ramp-down during the ITER non-activated phase [35]. Good control of the internal inductance is achieved with both ion species during the current ramp-up using a full bore plasma shape with early $\mathrm{X}$-point formation at 0.8 MA, equivalent to forming a diverted plasma at 4.5 MA in ITER. Early heating is required to keep $l_{\mathrm{i}}$ below 0.85 when using the fastest current ramp rate available $\left(0.36 \mathrm{MA} \mathrm{s}^{-1}\right)$, still maintaining an MHD stable plasma up to $q_{95}=3$ with a transition to H-mode which in JET deuterium discharges occurs at $7-9 \mathrm{MW}$ and in helium at $8-11 \mathrm{MW}$. During the current ramp-down the plasma inductance can be maintained 


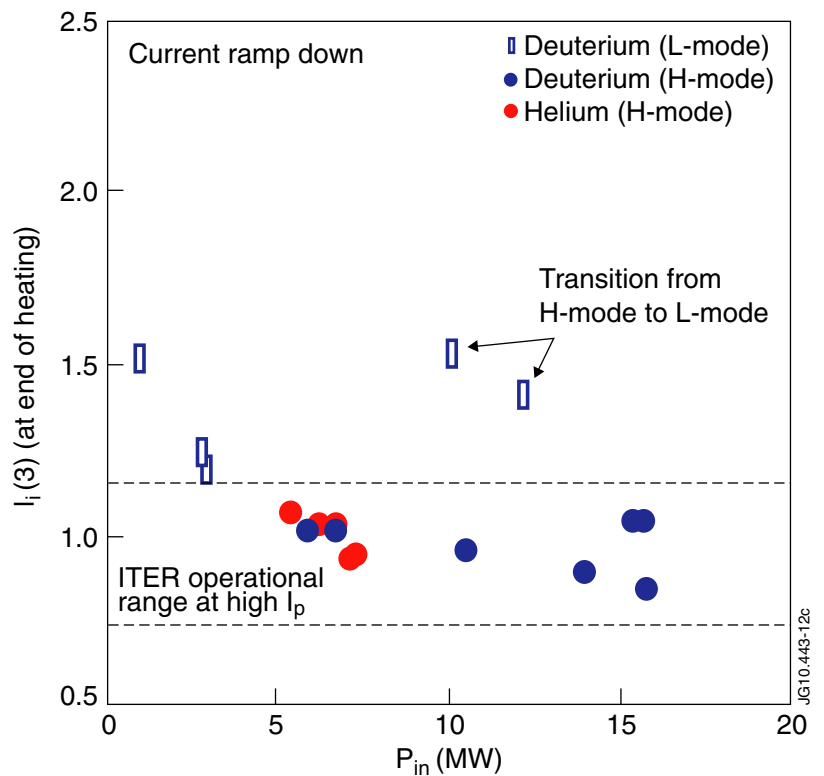

Figure 12. Plasma inductance $l_{\mathrm{i}}(3)$ at the end of the additional heating phase after current ramp down to half the flat-top current, plotted against applied heating power.

within the ITER limits by remaining in H-mode (figure 12). Some discharges using a fast ramp down rate of $0.5 \mathrm{MA} \mathrm{s}^{-1}$ do not remain in $\mathrm{H}$-mode despite heating powers well above the normal $\mathrm{L}-\mathrm{H}$ threshold powers as indicated in figure 12. If heating is not available, simultaneous control of the internal inductance and avoidance of flux consumption can, however, be achieved by combining an appropriate ramp-down rate with a strong reduction in plasma elongation to reduce the vertical instability growth rate. Apart from a higher flux consumption for helium discharges during plasma initiation deuterium and helium discharges are found to be very similar with respect to key requirements for ITER plasma control.

2.4.2. $L-H$ threshold power of helium plasmas. The $\mathrm{L}-\mathrm{H}$ threshold power of helium plasmas has been investigated and compared with that of matched deuterium plasmas by scanning the helium concentration from $1 \%$ to $87 \%$ in a set of low triangularity $(\delta \sim 0.25) 1.7 \mathrm{MA} / 1.8 \mathrm{~T}$ discharges [36]. In these plasmas, which had average densities in the range $n_{\mathrm{e}}=(2.5-2.9) \times 10^{19} \mathrm{~m}^{-3}$, the $\mathrm{L}-\mathrm{H}$ threshold power was found to be around $4 \mathrm{MW}$, or 1.2-1.4 times that predicted by the Martin08 scaling [19], with little dependence on the helium concentration (figure 13). This result is in line with recent ASDEX Upgrade findings [37] but different from what has been found at DIII-D [38] and also in previous JET studies [39] where the threshold power was found to be about $40 \%$ higher in helium. The earlier JET studies were, however, performed at lower plasma densities $\left(n_{\mathrm{e}}=(1-1.5) \times 10^{19} \mathrm{~m}^{-3}\right)$ and a significantly higher threshold $(>60 \%)$ was also found at lower density $\left(n_{\mathrm{e}}=2.1 \times 10^{19} \mathrm{~m}^{-3}\right)$ in these latest studies. The threshold power for transition from type-III to type-I ELMs was investigated separately in matched high triangularity $(\delta \sim 0.4) 1.7 \mathrm{MA} / 1.8 \mathrm{~T}$ discharges. This threshold was also found to be similar for both ion species, 6.7-9.3 MW for deuterium $\left(P_{\mathrm{I}-\mathrm{III}} / P_{\mathrm{Martin} 08}=1.2-1.8\right)$ and $7.5-9.3 \mathrm{MW}$ for

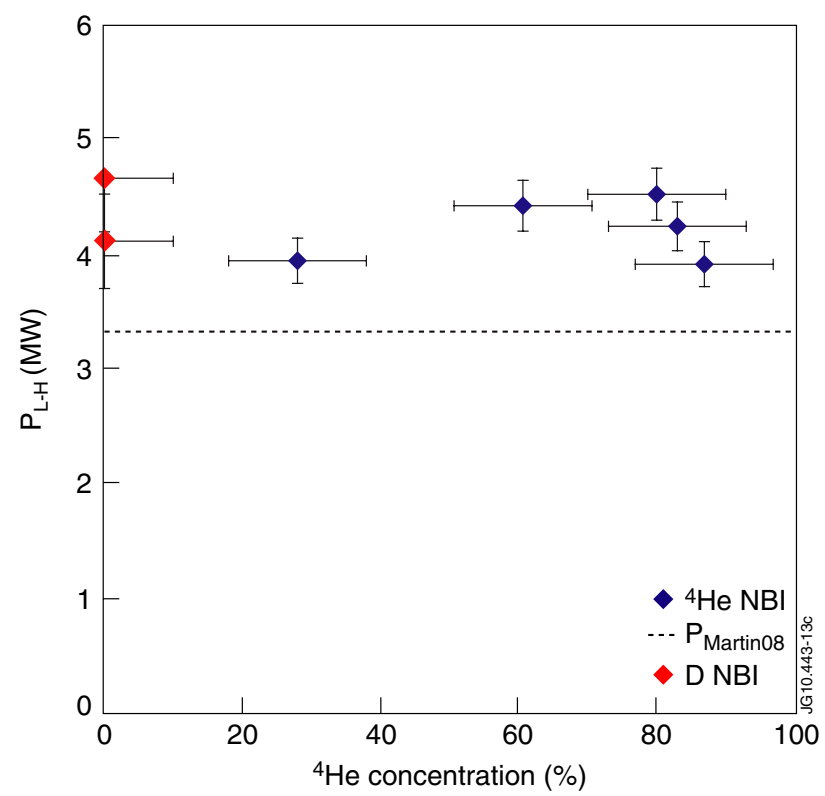

Figure 13. L-H threshold power versus helium concentration for plasmas heated by helium (blue) and deuterium (red) NBI in the density range $n_{\mathrm{e}}=(2.5-2.9) \times 10^{19} \mathrm{~m}^{-3}$. The dashed line indicates the threshold power according to the Martin08 scaling.

helium $\left(P_{\mathrm{I}-\mathrm{III}} / P_{\text {Martin08 }}=1.4-1.6\right)$. Scaled to the ITER halffield baseline scenario $\left(7.5 \mathrm{MA} / 2.65 \mathrm{~T}, n_{\mathrm{e}} / n_{\mathrm{GW}}=85 \%\right)$ using the Martin08 scaling the $\mathrm{L}-\mathrm{H}$ threshold power in helium should be in the range 30-42 MW (or 20-65 MW for an appropriately chosen $95 \%$ confidence interval). The threshold power for type-I to type-III ELMs would correspondingly be 42-48 MW (23-86 MW). These ranges are largely consistent with the design levels of ITER auxiliary heating powers.

\subsubsection{Helium plasma H-mode and power exhaust physics.} In the high triangularity type-I ELMy H-mode the energy confinement in helium (65-80\% purity) normalized to the IPB98 $(y, 2)$ scaling law was found to be around $60-80 \%$ of the confinement in the equivalent deuterium plasmas. Edge pedestal measurements revealed that the pedestal pressure in helium was around $70 \%$ of that in deuterium, although the pedestal widths were found to be similar, $2.1 \pm 0.5 \mathrm{~cm}$ in helium and $2.5 \pm 0.5 \mathrm{~cm}$ in deuterium (figure 14). Increased edge recycling, due to the lower efficiency of helium pumping, and impurity accumulation was observed in the helium plasmas and could be part of the explanation for the lower confinement.

Power exhaust studies of type-I ELMy H-modes also showed differences between deuterium and helium operation that need to be taken into account when drawing conclusions from the ITER non-activated operation in helium for future DT operation. Figure 15 shows the temporal and radial heat load profiles during a typical, medium size $(\Delta W / W \sim 4-5 \%)$ type-I ELM on the outer divertor target (which receives most of the average power) measured using infrared thermography in comparable deuterium and helium plasmas [40]. Compared with deuterium the inter-ELM heat load profile is significantly broader in helium, integral width $\lambda_{q}=5.4 \mathrm{~cm}$ compared with $\lambda_{q}=3.7 \mathrm{~cm}$ in deuterium. Since most of the energy reaches the target in the inter-ELM phase the average profile is also 


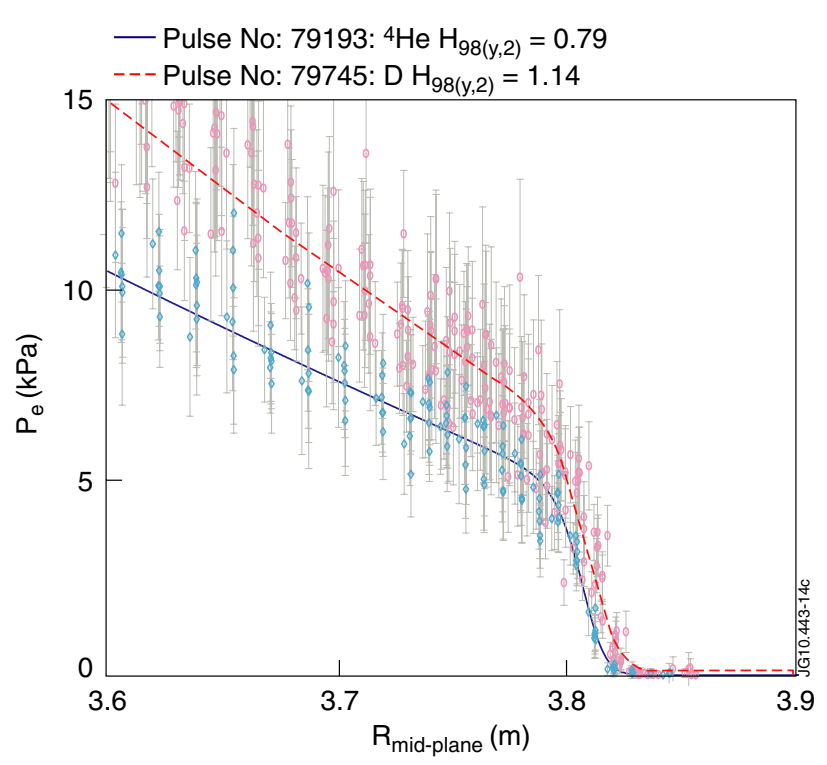

Figure 14. Plasma electron pressure profiles measured by HRTS for a pair of 1.7 MA/1.8 T high triangularity helium (blue) and deuterium (red) discharges with similar heating powers of $9.0 \mathrm{MW}$ (helium) and 10.4 MW (deuterium). Solid lines represent the fitted profiles.

broader in helium, leading to lower peak heat loads. While the time-integrated ELM heat load profiles are similar for the two species the power arrival time scale for ELMs in helium plasmas is significantly longer than in deuterium.

\section{Plasma transport and core stability}

\subsection{Momentum transport and intrinsic rotation}

Plasma rotation is well known to have beneficial effects on MHD modes, such as resistive wall modes (RWMs) and NTMs, and sheared plasma rotation is an important factor in plasma turbulence stabilization. The combination of sheared rotation and low magnetic shear for example appears to play a role in the improved core ion confinement observed in hybrid and advanced tokamak scenarios, with normalized ion temperature gradient lengths up to eight observed in the fastest rotating hybrid discharges on JET [26]. In light of the low external momentum input of the ITER 1 MV NBI system, a robust understanding of momentum transport and intrinsic momentum sources and sinks is crucial.

3.1.1. Momentum pinch and Prandtl number. The radial profiles of the inward momentum pinch and Prandtl number have been determined on JET [41] using modulated NBI powers and torques and compared with linear gyro-kinetic code predictions using GKW [42] and GS2 [43]. Quantitative agreement is found in the dependence of the pinch number, $R v_{\text {pinch }} / \chi_{\phi}$ (with $R$ the torus major radius, $v_{\text {pinch }}$ the pinch velocity and $\chi_{\phi}$ the toroidal momentum diffusivity), and the diffusive Prandtl number, $P_{r}=\chi_{\phi} / \chi_{\mathrm{i}}$, on the inverse density gradient length, $R / L_{n}=R|\nabla n| / n$. The dependence on other parameters is weak, and neither $R v_{\text {pinch }} / \chi_{\phi}$ nor $P_{r}$ depends on collisionality. $R v_{\text {pinch }} / \chi_{\phi}$ is found to be between 3 and 5 around the plasma mid-radius ( $r / a=0.4-0.8)$, only increasing above 5 for $R / L_{n}>3$. $P_{r}$, which does not depend significantly on any of the parameters scanned, is typically $1.5-2$ at the plasma mid-radius and increases with plasma minor radius.

3.1.2. Intrinsic rotation studies. In JET plasmas with normal toroidal magnetic field ripple, $\delta_{B_{\mathrm{T}}}=0.08 \%$, the intrinsic toroidal rotation in the absence of significant momentum injection by NBI is always small, $\omega_{\phi}< \pm 10 \mathrm{krad} \mathrm{s}^{-1}$, also in ICRH-dominated H-mode plasmas with $\beta_{\mathrm{N}}$ up to 1.3 [44]. This is in conflict with the Rice multi-machine scaling law for the intrinsic rotation, which predicts an Alfvén-Mach number an order of magnitude larger [45]. At the ITER ripple level, $\delta_{B_{\mathrm{T}}}=0.5 \%$, the JET intrinsic rotation is near zero. At higher toroidal field ripple the edge rotation is near-zero and the core is rotating in the counter-current direction, faster in plasmas with type-III than with type-I ELMs (figure 16). A separate study has analysed the relative loss of toroidal momentum to plasma energy associated with ELMs, showing that the momentum losses are consistently larger than the energy losses (figure 17). The losses of momentum are observed to penetrate deeper into the plasma during large type-I ELMs than the losses of energy, $r / a=0.65$ as compared with $r / a=0.8$. As a result, the timeaveraged toroidal rotation at the top of the pedestal decreases with increasing ELM frequency.

\subsection{Fast particle/burning plasma physics}

A comprehensive set of fast ion diagnostics (neutron and $\gamma$-ray cameras and spectrometers, neutral particle analyser (NPA)) and lost ion diagnostics (gyroradius and pitch-angle resolved scintillator probe, thin-foil Faraday cups and an activation probe) coupled with a flexible heating system capable of producing fast ions in the $\mathrm{MeV}$ energy range and the large machine size and high plasma current that allow them to remain confined make JET particularly well suited for fast particle and burning plasma studies [46].

In the advanced tokamak discharges with high $\beta_{\mathrm{N}}$ and $q_{0}>1.5$ described above [28], plasma disruptions preceded by strong $m / n=2 / 1$ MHD modes were found to be accompanied by large fast ion bursting losses during the thermal quench (TQ) (figure 18) [47]. Scintillator probe measurements indicate that these losses are consistent with trapped ions accelerated by ICRH. Bursts of metallic impurity influx were also observed in connection with the losses. These observations are consistent with a theory [48] for the redistribution of energetic trapped ions by interaction with a pressure driven $m / n=2 / 1 \mathrm{kink}$ mode which also leads to internal magnetic reconnection, visible in figure 18 as an abrupt change in plasma internal inductance.

Losses of high-energy $(0.5-4 \mathrm{MeV})$ protons accelerated by ICRH were also observed with the $2 \mathrm{D}$ scintillator probe in recent JET experiments with low-frequency $(9-14 \mathrm{kHz})$ fishbones driven unstable by NBI ions $(80-130 \mathrm{keV})[49,50]$. The losses were enhanced a factor of 10-20 with respect to MHD-quiescent levels and were found to increase quadratically with the mode amplitude. Due to the difference in frequencies between the fishbone modes and the orbit periodic motions of the lost ions these losses could not have been caused by resonant interactions [51]. Theory, 

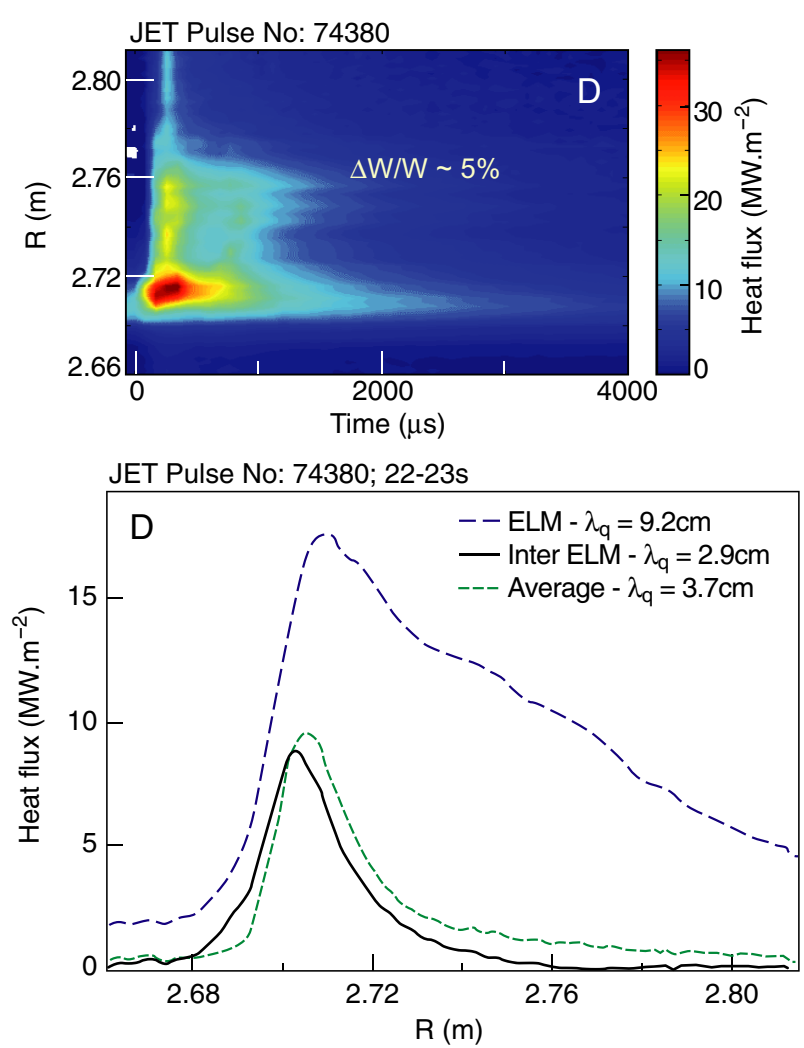
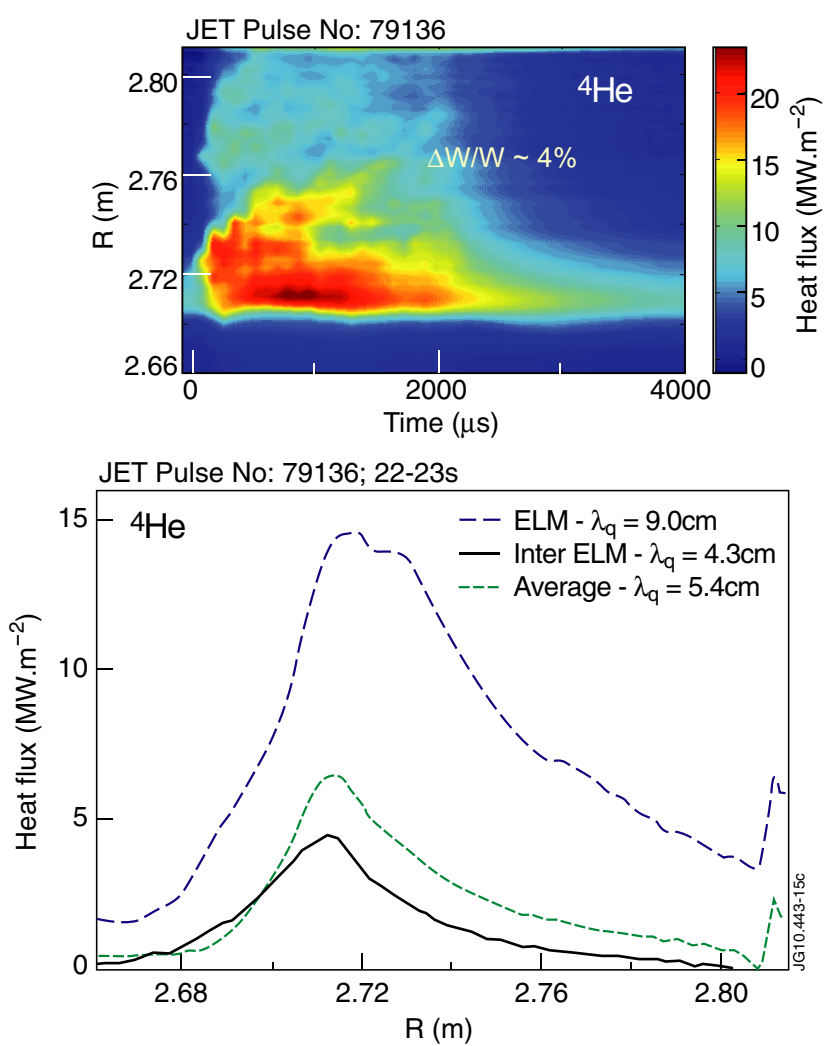

Figure 15. Top frames: temporal evolution of the heat load profiles during a typical, medium size $(\Delta W / W \sim 4-5 \%)$ ELM on the outer divertor target in comparable deuterium and helium plasmas measured using infrared thermography. Bottom frames: time-integrated radial profiles of the ELM, inter-ELM and average heat loads and their corresponding integral widths $\lambda_{q}$.

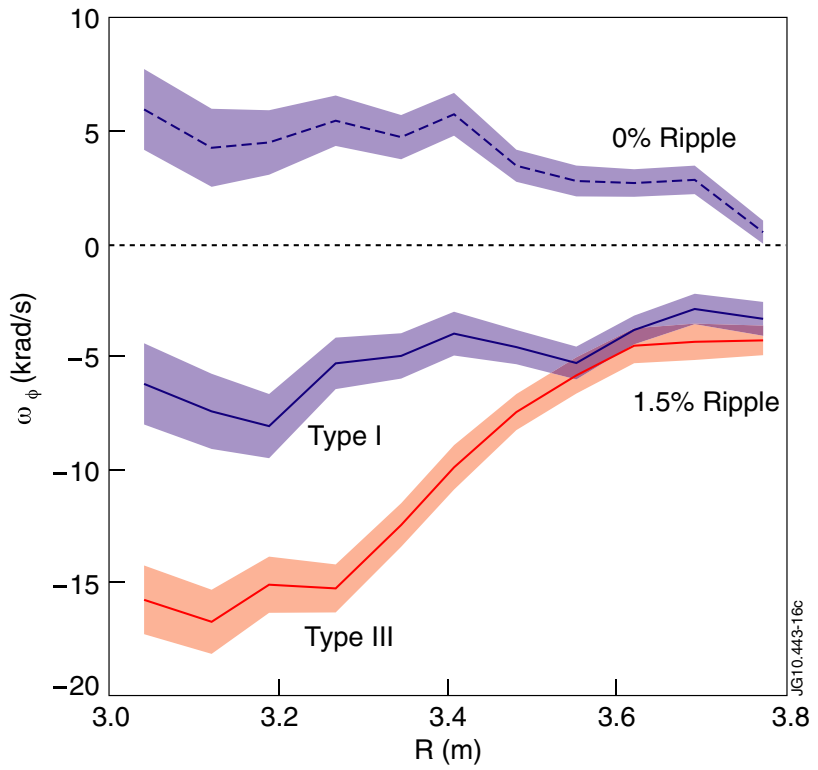

Figure 16. Toroidal rotation profiles for ICRF-heated H-mode $1.5 \mathrm{MA} / 2.2 \mathrm{~T}$ plasmas with $0.08 \%$ toroidal field ripple and $P_{\text {ICRH }}=3.1 \mathrm{MW}$ (blue) and $1.5 \%$ ripple, $P_{\text {ICRH }}=2.9 \mathrm{MW}$ for both the type-I (blue) and type-III (red) ELM phase.

however, suggests [52] that the loss of toroidal symmetry caused by the fishbone can also cause non-resonant losses of high-energy ions and $\alpha$-particles. Detailed integrated MISHKA [53], HAGIS [54] and SELFO [55] modelling with

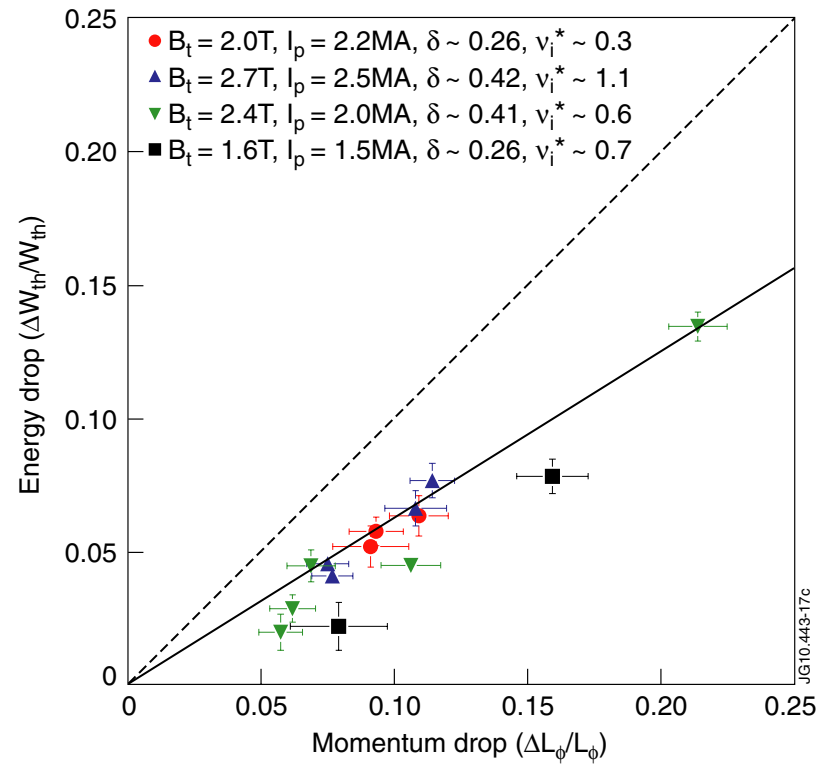

Figure 17. Normalized thermal energy drop versus normalized momentum drop per ELM.

a detailed synthetic scintillator probe model [50] confirm that the observed losses result from non-resonant interactions. Very good agreement is found between the measured and simulated energy and pitch-angle distributions as well as the temporal evolution of the losses during a fishbone cycle. 

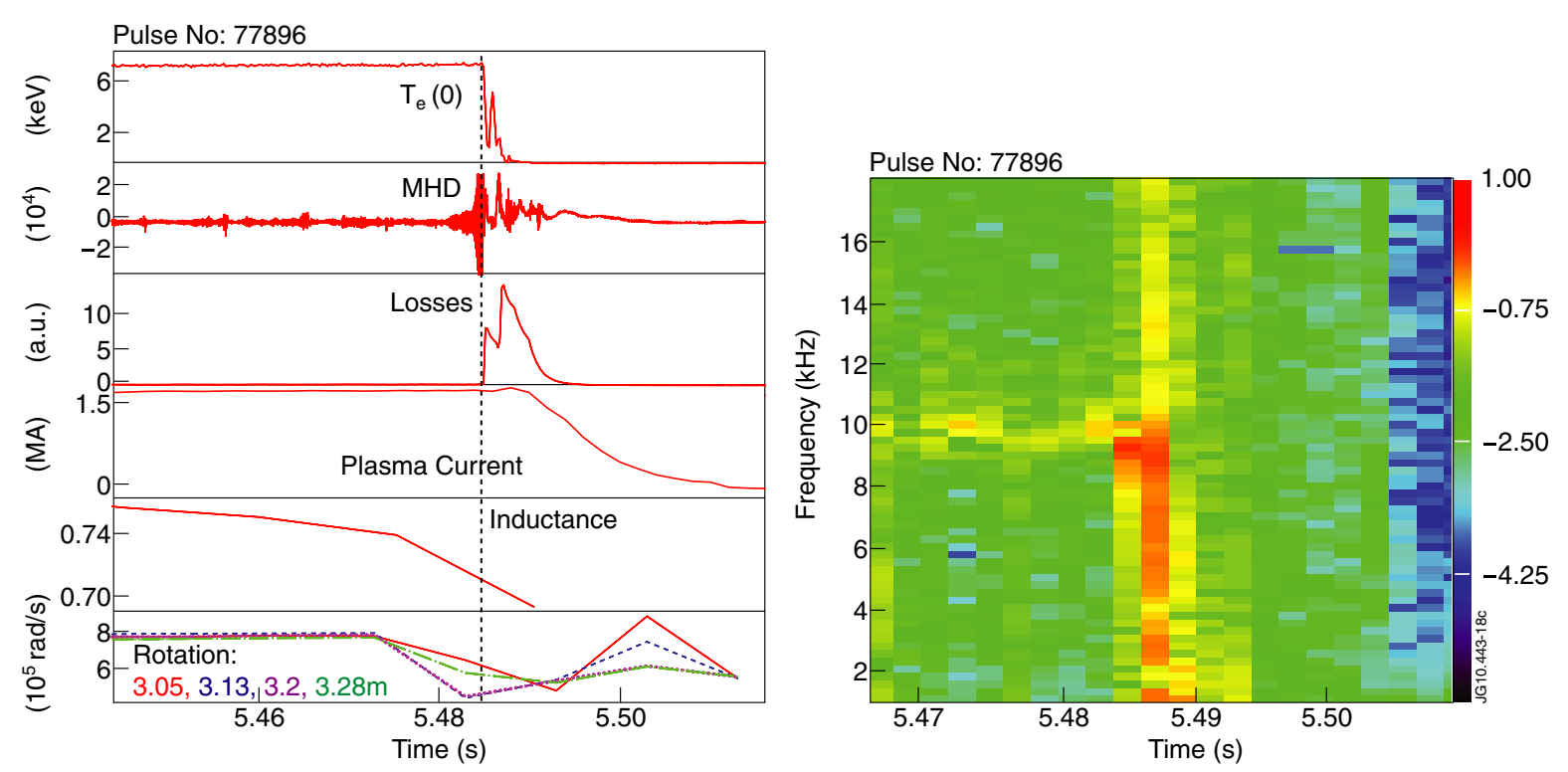

Figure 18. Plasma parameter time traces and magnetic spectrogram showing an $m / n=2 / 1$ mode followed by a disruption with strong losses of fast ions during the TQ measured using the scintillator probe.

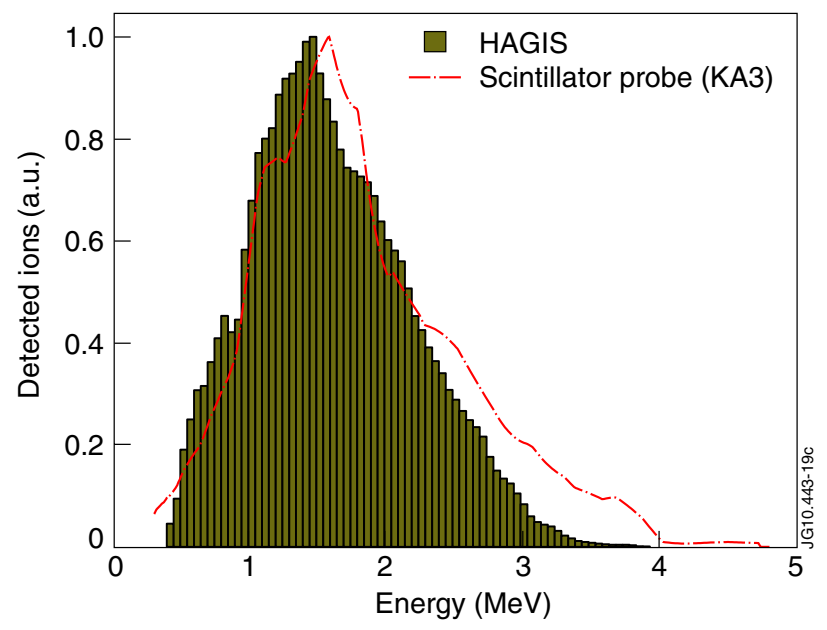

Figure 19. Energy distribution of proton losses arriving at the scintillator detector predicted by HAGIS, convoluted with the instrumental function of the diagnostic, and energy distribution of losses measured by the scintillator probe.

The measured and simulated lost ion energy distributions are shown in figure 19. The losses are found to originate from orbit stochastic diffusion of trapped protons near the plasma boundary or from counter-passing protons deep in the plasma core which transform into unconfined trapped orbits under the influence of the fishbone. Nearly all of the fast ion losses take place in the early growth phase of the fishbone cycle, reaching their maximum well ahead of the magnetic perturbation peak.

\subsection{Sawtooth stability control}

Effective sawtooth control has been demonstrated with ${ }^{3} \mathrm{He}$ minority ICRH with toroidally directed antenna spectra and the resonance tangential to the $q=1$ surface [56]. Since the minority ion current drive for this scenario is expected to be negligible in JET (and in ITER) due to the electron drag current, this demonstrates the direct kinetic response of highly energetic ions on the internal kink mode. The effect is explained by fast ions with wide drift orbits intersecting the $q=1$ surface predominantly on the high-field side (good magnetic curvature) or low-field side (bad magnetic curvature) due to asymmetric parallel velocity distributions [57]. Using $4 \mathrm{MW}$ of ICRH the sawtooth period could be decreased $\left(-90^{\circ}\right.$ antenna phasing) or increased $\left(+90^{\circ}\right.$ antenna phasing) by more than a factor of 2 (figure 20). This direct effect of fast ions on the sawtooth stability is encouraging for ITER, where the ability of the ICRH system to control the magnetic shear by ion cyclotron current drive (ICCD) is expected to be weak [58].

\section{First wall power load studies}

\subsection{Preparations for operation with the ILW}

The ILW project sees the replacement of all CFC PFCs with beryllium for the first wall (solid Be and $8 \mu \mathrm{m}$ Be-coated inconel) and tungsten in the divertor (figure 21). Most of the divertor is covered by $10-15 \mu \mathrm{m} \mathrm{W}$-coated CFC tiles with 20-25 $\mu \mathrm{m}$ W-coated CFC used for tiles 6 and 7 due to the higher erosion from physical sputtering expected in the inner divertor [7,8] (figure 22). Based on extrapolations of the erosion of similar, but thinner, W coatings installed during recent campaigns the lifetime of the coated divertor tiles is expected to be in the order of 100000 plasma seconds [59]. Bulk tungsten is used for the horizontal tile for the outer strike point in high triangularity high performance scenarios $[9,10]$. The characterization and exploitation of the wall will form the basis for the JET programme in the coming years and, since the beryllium and tungsten material combination has never before been tested in a fusion device, the scientific exploitation of the new wall will start from day one. A significant part of the recent JET programme has therefore been devoted to (i) the development of techniques to ensure the safe operation with the new wall and (ii) provision for reference plasmas to allow 

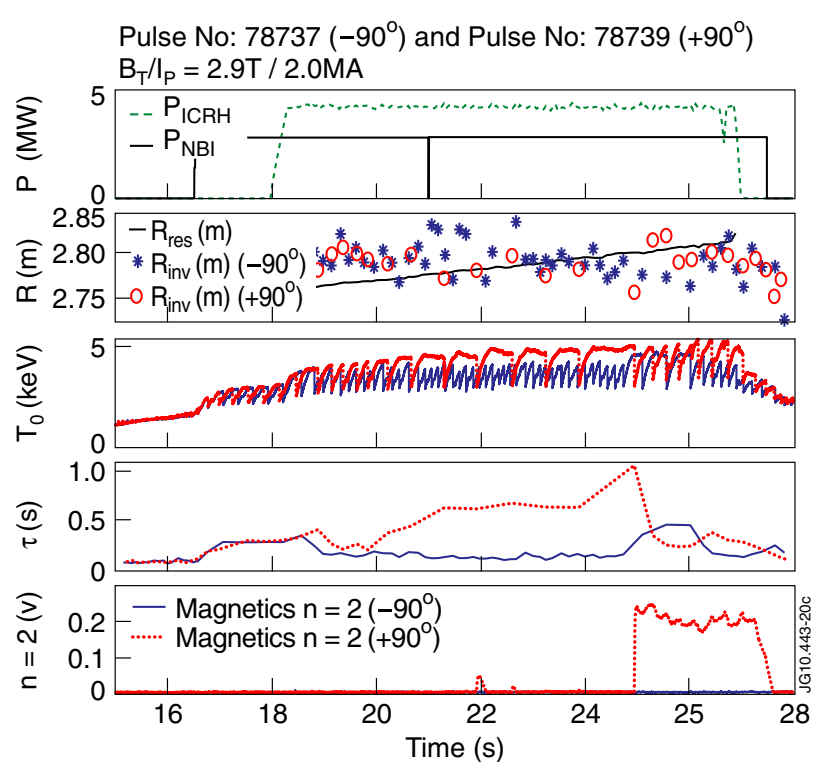

Figure 20. Sawtooth control relying on fast particles with ${ }^{3} \mathrm{He}$ minority ICRH. After the application of ICRH the sawtooth period $\tau(\mathrm{s})$ decreased $\left(-90^{\circ}\right.$ antenna phasing $)$ or increased $\left(+90^{\circ}\right.$ antenna phasing) by more than a factor of 2 . With the $+90^{\circ}$ phasing an NTM is eventually triggered by a $1 \mathrm{~s}$ sawtooth, as indicated by the $n=2$ magnetic signal.
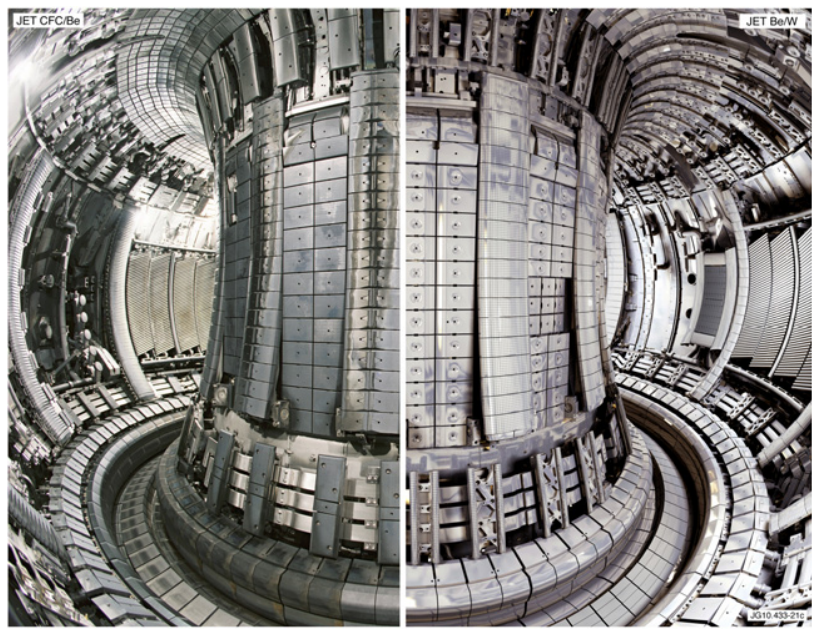

Figure 21. Previous JET CFC/Be wall (photograph, left) and the new ITER-like Be wall and $\mathrm{W}$ divertor (3D render, right).

exhaustive comparisons between the performance of carbon and metallic walls [60]. It is expected that compared with carbon the new wall materials will demonstrate a significant beneficial impact on key ITER issues such as fuel retention and the lifetime of PFCs.

Key for the successful operation with the tungsten divertor will be to replace the missing intrinsic carbon radiation with extrinsic impurity seeding to reduce the peak power loads in the divertor and to keep the divertor temperature below $10 \mathrm{eV}$, necessary to minimize tungsten physical sputtering by impurities in order to both ensure the integrity of the tungsten coatings and minimize the influx and potential accumulation of tungsten in the plasma core. As a precaution against possible recrystallization of tungsten and carbidization of the tungstenCFC interface a tungsten temperature limit of $1200^{\circ} \mathrm{C}$ will be

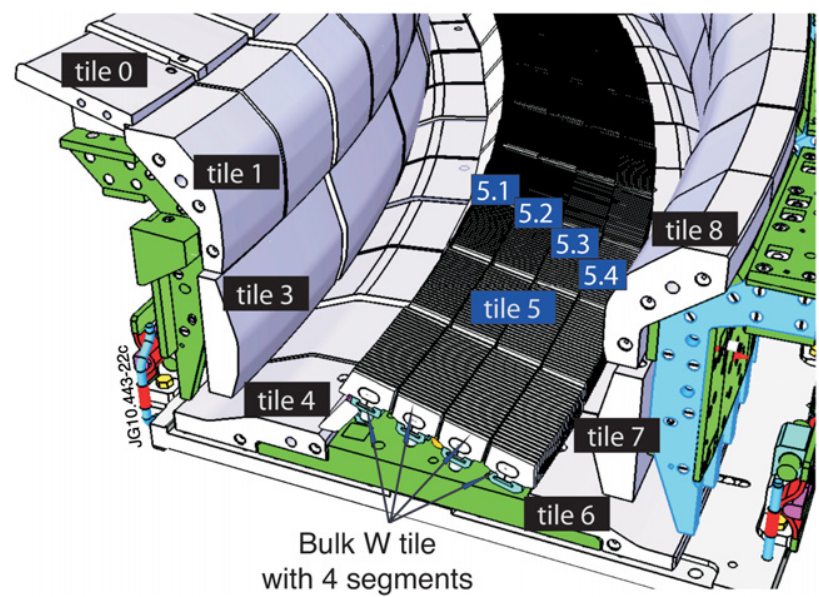

Figure 22. The new all-tungsten divertor. Tile 5, for the outer strike point, is made up of four segments of stacked bulk tungsten lamellae, other tiles are 10-25 $\mu \mathrm{m}$ tungsten-coated CFC.

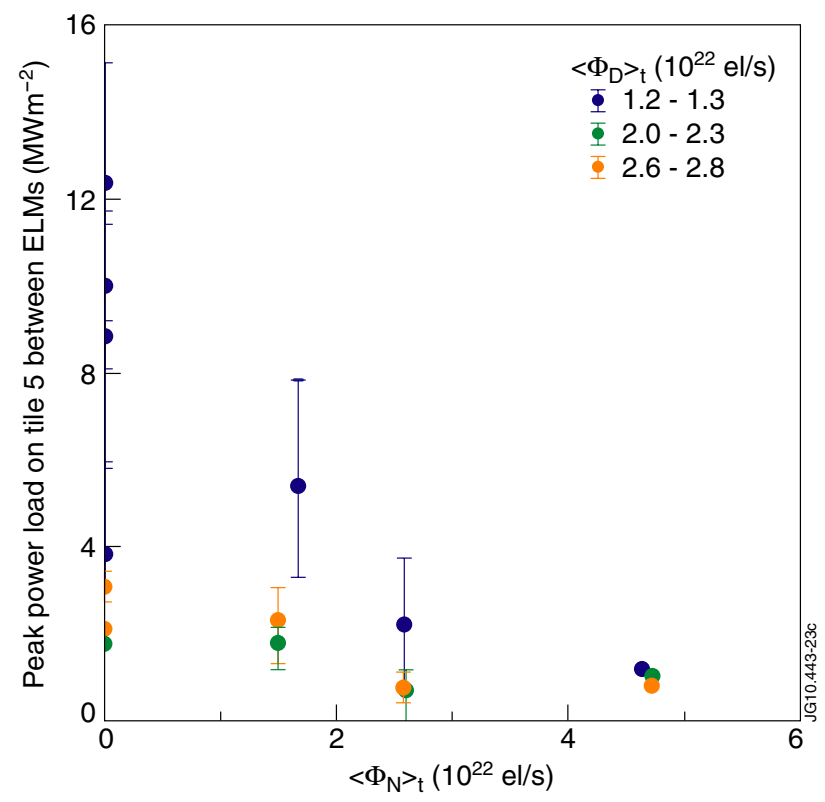

Figure 23. Peak power loads at the outer strike point between ELMs as a function of the time-averaged nitrogen seeding rate $\left\langle\Phi_{N}\right\rangle_{t}$ for various deuterium fuelling rates $\left\langle\Phi_{\mathrm{D}}\right\rangle_{\mathrm{t}}$. Deuterium was injected from the private flux region and nitrogen from the outer divertor.

imposed for the early exploitation of the wall, corresponding to power loads around $6 \mathrm{MW} \mathrm{m}^{-2}$ for $10 \mathrm{~s}$. The temperature limit will be raised to 1600 and $2200{ }^{\circ} \mathrm{C}$ as operational experience is gained. Systematic fuelling and impurity seeding scans, using nitrogen and neon due to their favourable radiation characteristics comparable to carbon, have been performed in a 2.5 MA/2.7 T $\left(q_{95}=3.5\right)$ type-I ELMy H-mode scenario with $16 \mathrm{MW}$ of auxiliary heating. Figure 23 shows the peak power load at the outer strike point in between ELMs for various combinations of deuterium fuelling from the private flux region and nitrogen seeding from the outer divertor. Already pure fuelling, with the aid of intrinsic carbon radiation which, however, is expected to be largely absent with the new wall, lead to a significant reduction in the peak power load from $\sim 13$ to $\sim 4 \mathrm{MW} \mathrm{m}^{-2}$. Moderate fuelling $\left(1.85 \times 10^{22} \mathrm{el} \mathrm{s}^{-1}\right)$ 


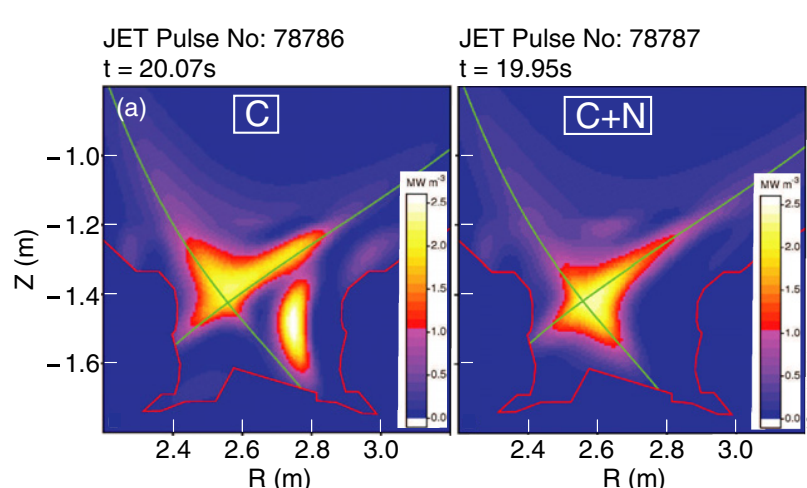

(b)

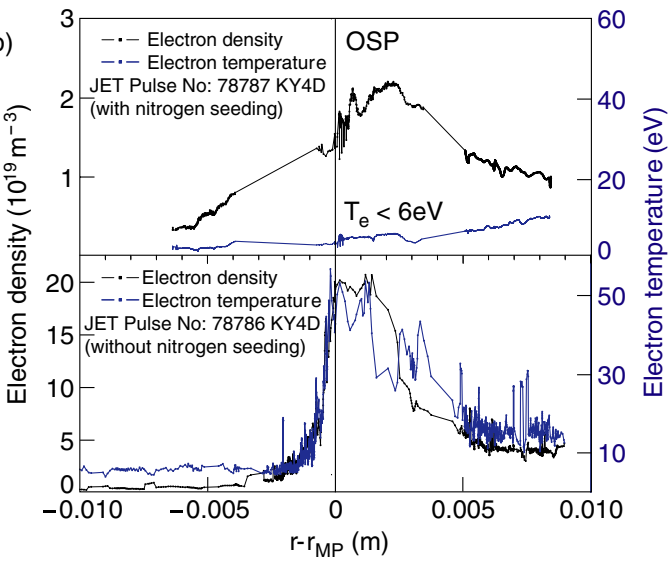

Figure 24. (a) Inter-ELM radiation pattern without and with nitrogen seeding showing the radiation with nitrogen to be concentrated in the X-point region. $(b)$ Electron temperatures and densities mapped to the outer mid-plane.

and seeding $\left(1.5 \times 10^{22} \mathrm{el} \mathrm{s}^{-1}\right)$ lead to tungsten divertor compatible peak power loads below $2 \mathrm{MW} \mathrm{m}^{-2}$ and acceptable type-I ELMs for a moderate reduction in confinement of $10 \%$ compared with the unfuelled scenario. Increasing the nitrogen seeding to $3.55 \times 10^{22} \mathrm{el} \mathrm{s}^{-1}$ leads to complete detachment of the inner divertor leg, as diagnosed by Langmuir probes and divertor spectroscopy, and partial detachment of the outer leg for a negligible reduction in confinement, $H_{98(y, 2)}=0.95$ compared with $H_{98(y, 2)}=0.96$ for the fuelled $\mathrm{H}$-mode. Figure 24 shows the bolometric reconstruction of the divertor radiation between two ELMs with and without nitrogen seeding and the corresponding electron densities and temperatures mapped to the outer mid-plane. Nitrogen seeding reduces both the temperatures and densities by almost a factor of 10, demonstrating a loss of pressure and partial detachment of the outer strike point. The resulting low divertor electron temperature, $T_{\mathrm{e}}<6 \mathrm{eV}$, with nitrogen seeding would with the ILW ensure low levels of tungsten erosion by physical sputtering for long lifetimes of the tungsten coatings and low levels of tungsten impurity influx into the plasma core.

\subsection{ELMs and their amelioration}

The understanding and mitigation of ELMs are one of the main issues for reliable ITER operation. ITER will require reliable ELM control over a wide range of operating conditions and it is therefore essential to develop a suite of different ELM-mitigation techniques. On JET, ELM control studies

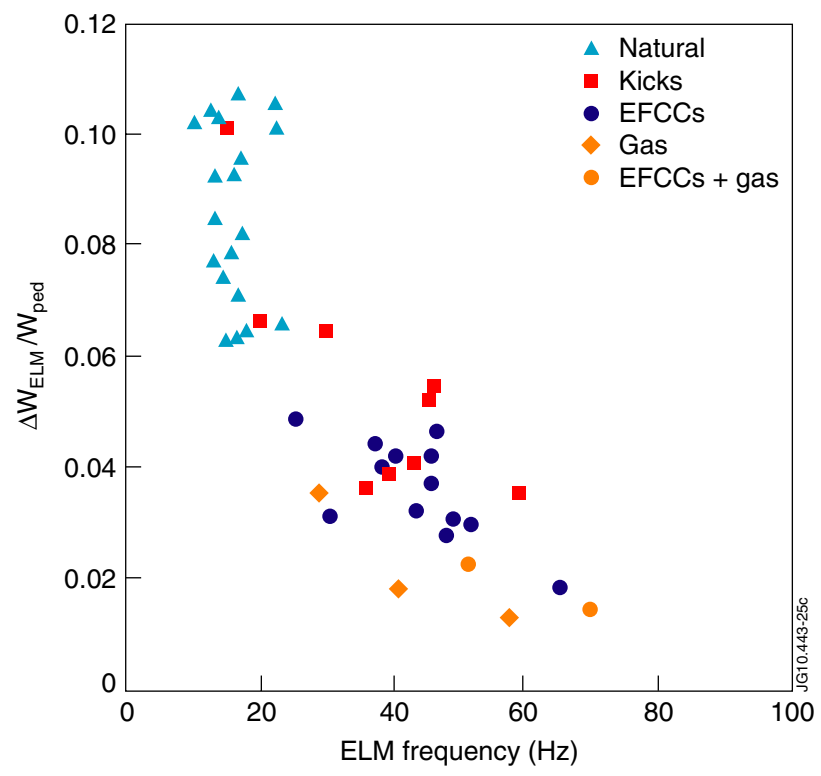

Figure 25. Normalized ELM energy loss (to the pedestal energy) versus ELM frequency (high $\delta, q_{95}=3.6-3.9, P_{\mathrm{NBI}}=10-12 \mathrm{MW}$ ).

using resonant magnetic perturbations (RMP) produced by the external error field correction coils (EFCCs), rapid radial field changes ('vertical kicks'), gas injection and pellet pacing have progressed towards establishing the necessary conditions for mitigation, the impact on the plasma confinement and the effect on the divertor heat loads [61-63].

4.2.1. ELM-mitigation studies with RMPs and vertical kicks. The application of EFCCs and kicks in high triangularity $(\delta=0.43) \mathrm{H}$-mode plasmas $\left(2 \mathrm{MA} / 2.2-2.4 \mathrm{~T}, q_{95}=3.6-3.9\right.$, $P_{\mathrm{NBI}}=7-12 \mathrm{MW}, P_{\mathrm{ICRH}}=1-2 \mathrm{MW}$ ) with low natural ELM frequencies, $f_{\mathrm{ELM}} \sim 7-15 \mathrm{~Hz}$, allows an increase in ELM frequency by a factor of 5 with vertical kicks and a factor of 3.5 with EFCCs [61]. The increase in ELM frequency is associated with a decrease in the normalized energy loss per ELM (figure 25). Notably, all ELM control methods (EFCCs, vertical kicks and gas) follow the same general trend in $\Delta W_{\mathrm{ELM}} / W_{\mathrm{PED}}$ with $f_{\mathrm{ELM}}$ and the mitigated ELMs can be sustained also at low pedestal collisionality. Both EFCCs and kicks are associated with a density pump-out which reduces the core density by $\sim 30 \%$. This can be compensated for by gas puffing, but at a cost in confinement. Toroidal rotation braking (up to 50\%) is observed and extends over the whole plasma column with EFCCs, whereas with kicks a $\sim 10 \%$ reduction in the edge rotation is found due to the increased losses of toroidal momentum at the higher ELM frequency, see section 3.1.2.

On DIII-D, in-vessel RMP coils producing an $n=$ 3 perturbation field allows type-I ELMs to be completely suppressed in narrow windows of the edge safety factor $\left(q_{95}=\right.$ 3.5-3.9 and $\left.q_{95} \sim 7.2\right)$ [64, 65]. On JET, ELM control studies with $n=1$ or $n=2$ perturbation fields induced by the EFCCs have not yet shown complete ELM suppression, even in plasmas with edge vacuum Chirikov parameters greater than $1\left(0.85\right.$ at $\left.\Psi^{1 / 2}=0.95\right)$ [66]. In low triangularity plasmas $(\delta \sim 0.2)$ a $q_{95}$ scan at fixed toroidal field $(1.84 \mathrm{~T})$ and low pedestal collisionality $\left(v^{*} \sim 0.1\right)$, however, shows a resonance 


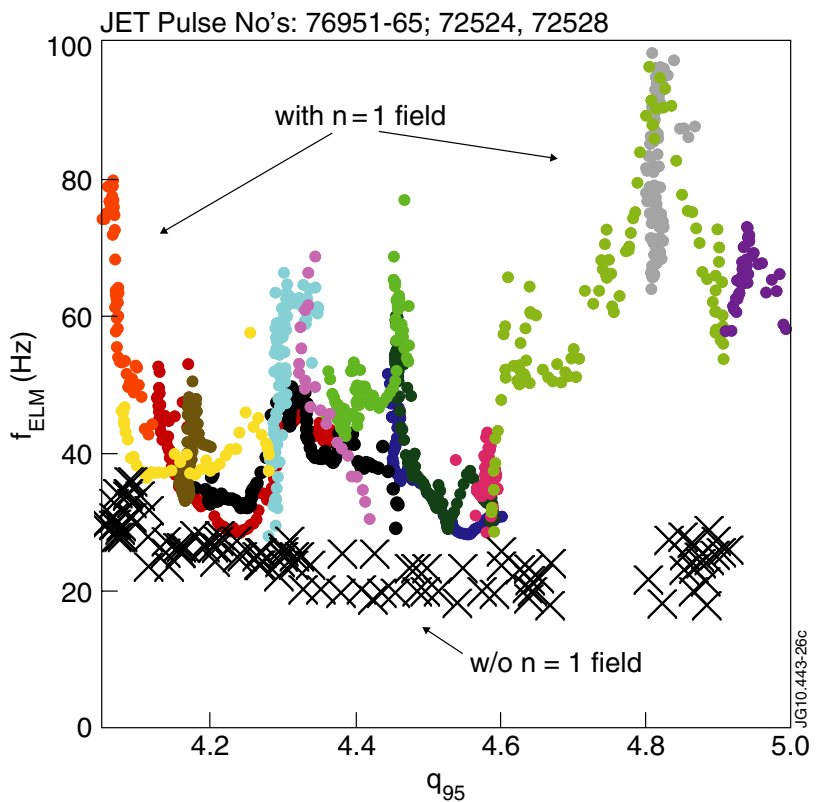

Figure 26. ELM frequency resonances in $q_{95}$ with $n=1$ fields applied by EFCCs.

effect, where the ELM frequency increases a factor of 4-5 at specific values of $q_{95}$ with $n=1$ fields applied. For non-resonant values of $q_{95}$ the ELM frequency only increases a factor of about 2 with the $n=1$ field applied [62,67] (figure 26). A similar effect is also found when an $n=2$ field is applied. This multiple resonance effect can be qualitatively predicted by a model in which the ELM width is determined by a localized relaxation to a profile which is stable to ideal external peeling modes [68].

4.2.2. ELM pellet pacing studies. Pellet pacing up to $10 \mathrm{~Hz}$ has been demonstrated in $2.0 \mathrm{MA} / 2.3 \mathrm{~T}\left(q_{95}=3.8\right)$ plasmas with $11 \mathrm{MW}$ of NBI heating using the fuelling section (nominally $2.2 \times 10^{21} \mathrm{D} /$ pellet, $200 \mathrm{~m} \mathrm{~s}^{-1}$ ) of the highfrequency pellet injector (HFPI) for low-field side injection. While the natural ELM frequency in these plasmas was also around $10 \mathrm{~Hz}$, the ELMs could be synchronized with the pellets - confirming the ELM pellet pacing technique on JET [63]. The minimum pellet size (and thereby the minimum unavoidable fuelling) required to trigger ELMs has been estimated from the $\mathrm{D}_{\alpha}$ signal from pellets with a large size scatter injected with the pacing section of the HFPI from the vertical high-field side (figure 27). If the $\mathrm{D}_{\alpha}$ pulse height is proportional to the pellet mass entering the plasma the trigger threshold is found to be $\sim 10^{19} \mathrm{D}$ or, using instead the total integral $\mathrm{D}_{\alpha}$ emission, $\sim 1.6 \times 10^{19} \mathrm{D}$. According to modelling, pellets of this size should penetrate to at least half the pedestal width [69]. Upgrades and improvements to the HFPI are being implemented and should allow further studies on pellet ELM pacing at frequencies significantly above the natural ELM frequency when JET restarts operation.

4.2.3. ELM-wetted area. Using a newly installed fast ( $86 \mu \mathrm{s})$ high-resolution $(1.7 \mathrm{~mm})$ infrared camera viewing tile 5 of the JET divertor (see figure 28) has allowed detailed studies on the ELM dynamics and in particular the inter-ELM and

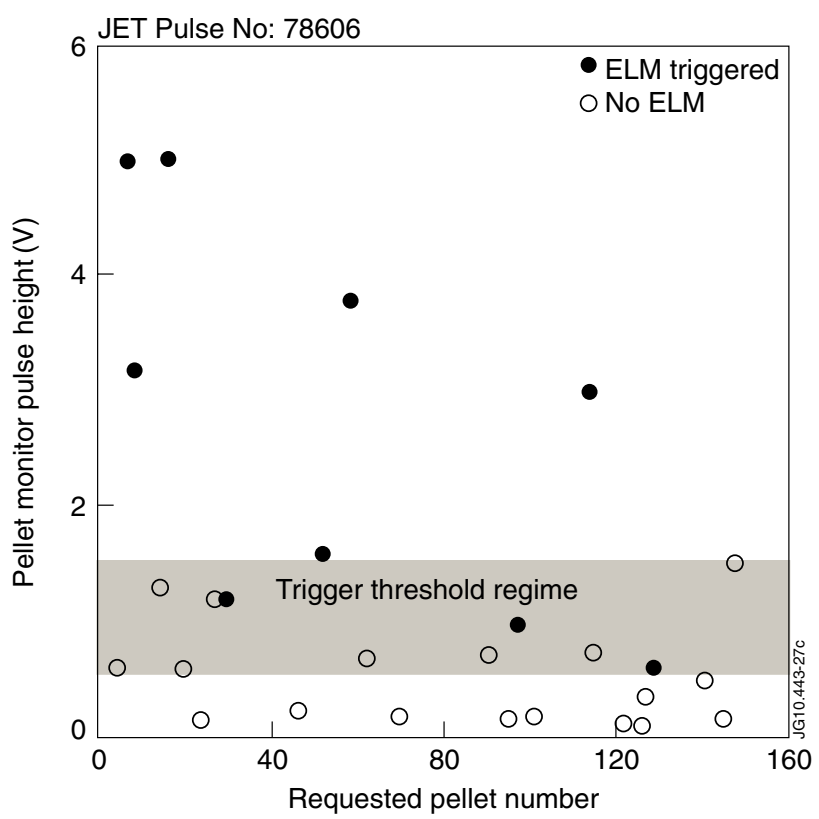

Figure 27. Pellet monitor $\left(D_{\alpha}\right)$ pulse height against pellet request number for a pellet train launched from the vertical high-field side. $1 \mathrm{~V}$ pulse height corresponds to $\sim 10^{19} \mathrm{D}$ /pellet.

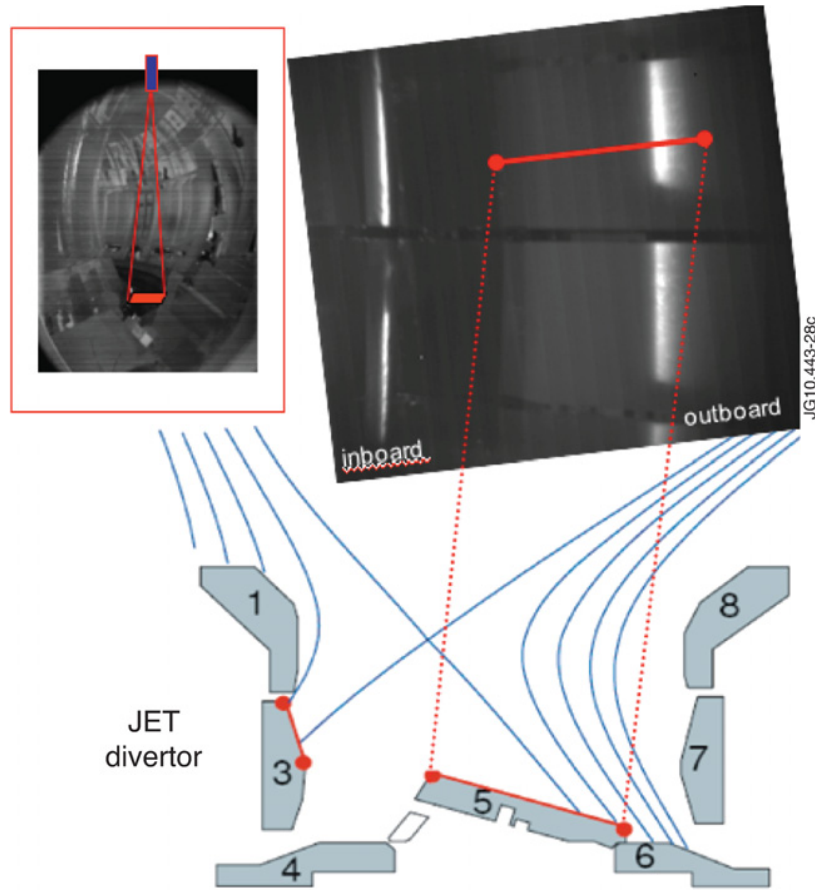

Figure 28. Setup of newly installed fast high-resolution IR camera viewing the JET divertor.

the ELM heat load profile wetted areas in high triangularity configurations $[40,70,71]$. Figure 29 shows the temporal evolution of the power arriving at the divertor target during an ELM crash and the ELM and inter-ELM radial heat load profiles. During the rapid rise in the ELM power load, which typically takes place on the ion-transit time scale, broadening of the ELM-wetted area is found which is characterized by distinct striations interpreted as footprints of plasma filaments ejected during the ELM crash. The number of striations appear 

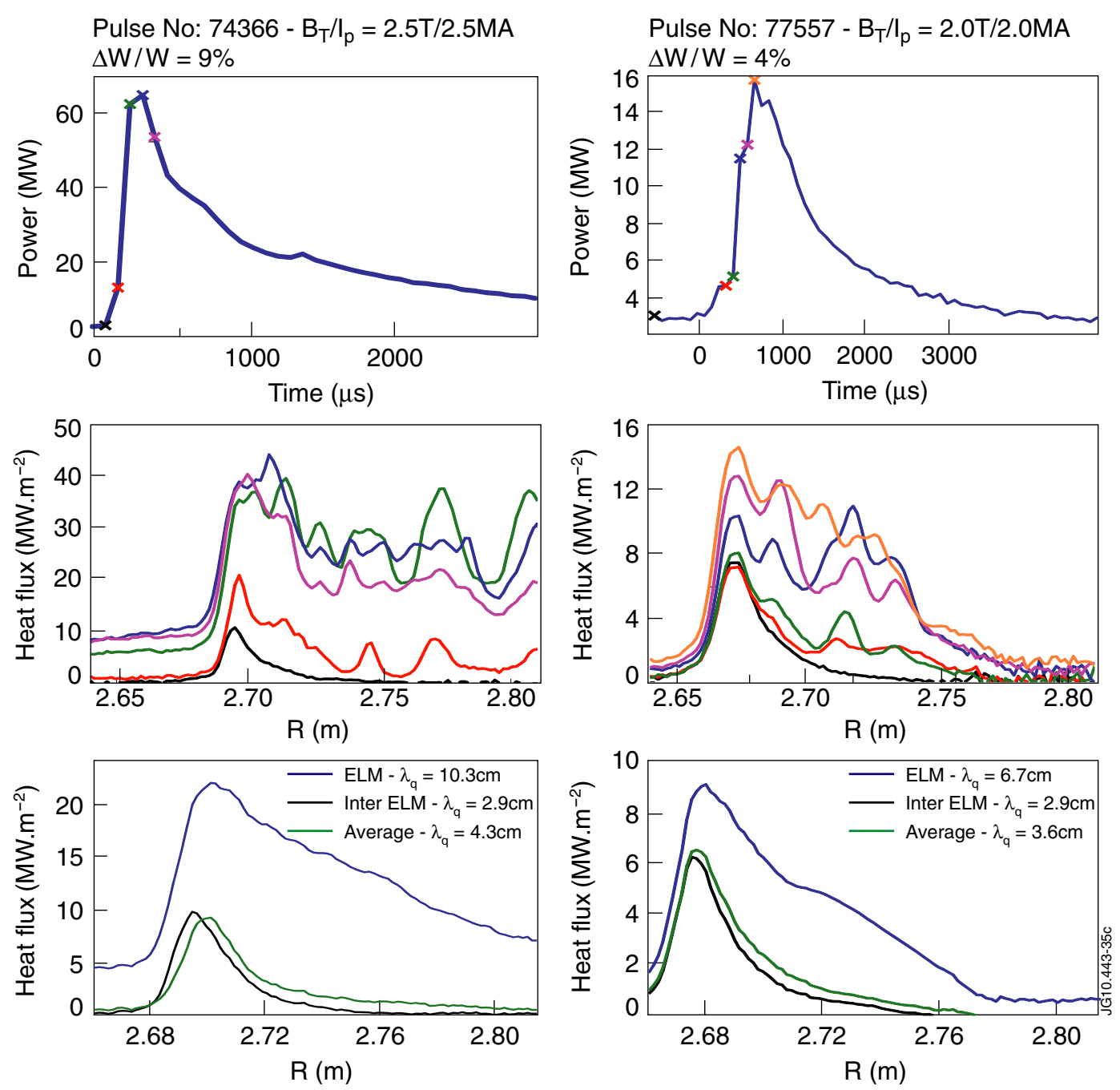

Figure 29. ELM dynamics for two different size ELMs, $\Delta W_{\mathrm{ELM}} / W_{\mathrm{dia}}=9 \%$ (left) and $\Delta W_{\mathrm{ELM}} / W_{\mathrm{dia}}=4 \%$ (right). Top frames show the temporal evolution of the power arriving at the target during the ELM crash, middle frames show a series of snapshots of the target heat load profiles for the time points indicated in the top frames and the bottom frames show the time-averaged radial heat load profiles.

to be independent of the input power, but increases from around 3-5 to 10-20 during the ELM rise phase [72]. From figure 29 it is evident that the ELM broadening increases with ELM size and this is also confirmed in a wider database of the ELMwetted area $\left(A_{\text {wet }}\right.$ defined as ratio between target-integrated power $P(\mathrm{~W})$ and the peak heat flux $Q_{\text {peak }}\left(\mathrm{W} \mathrm{m}^{-2}\right)$ ) (figure 30 ) and a similar ELM broadening is found in both natural as well as mitigated ELMs regardless of the mitigation method used $[61,71]$.

\subsection{Disruption studies for ITER}

The experimental disruption studies on JET have focussed on the understanding of asymmetric vertical displacement event (VDE) disruptions and on disruption amelioration by massive gas injection (MGI) as a means to reduce the impact of disruptions on the tokamak structure (electromagnetic forces from halo and eddy currents and localized heat loads from convection and runaway electrons) [73,74]. In addition, an extensive survey of all JET disruptions over the last decade

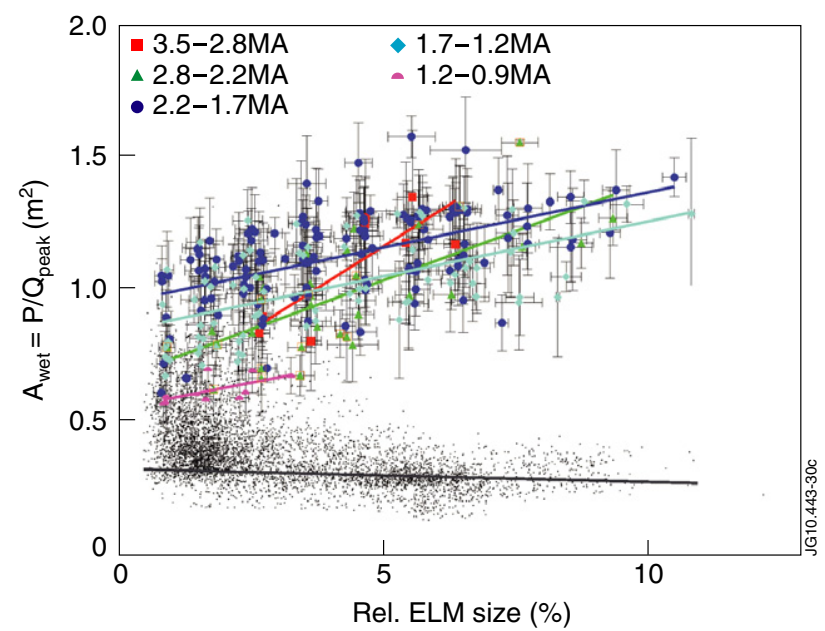

Figure 30. ELM-wetted area as a function of relative ELM size $\Delta W_{\mathrm{ELM}} / W_{\mathrm{MHD}}$ Different colours denote different plasma current intervals with solid lines the linear fits in respective intervals. 


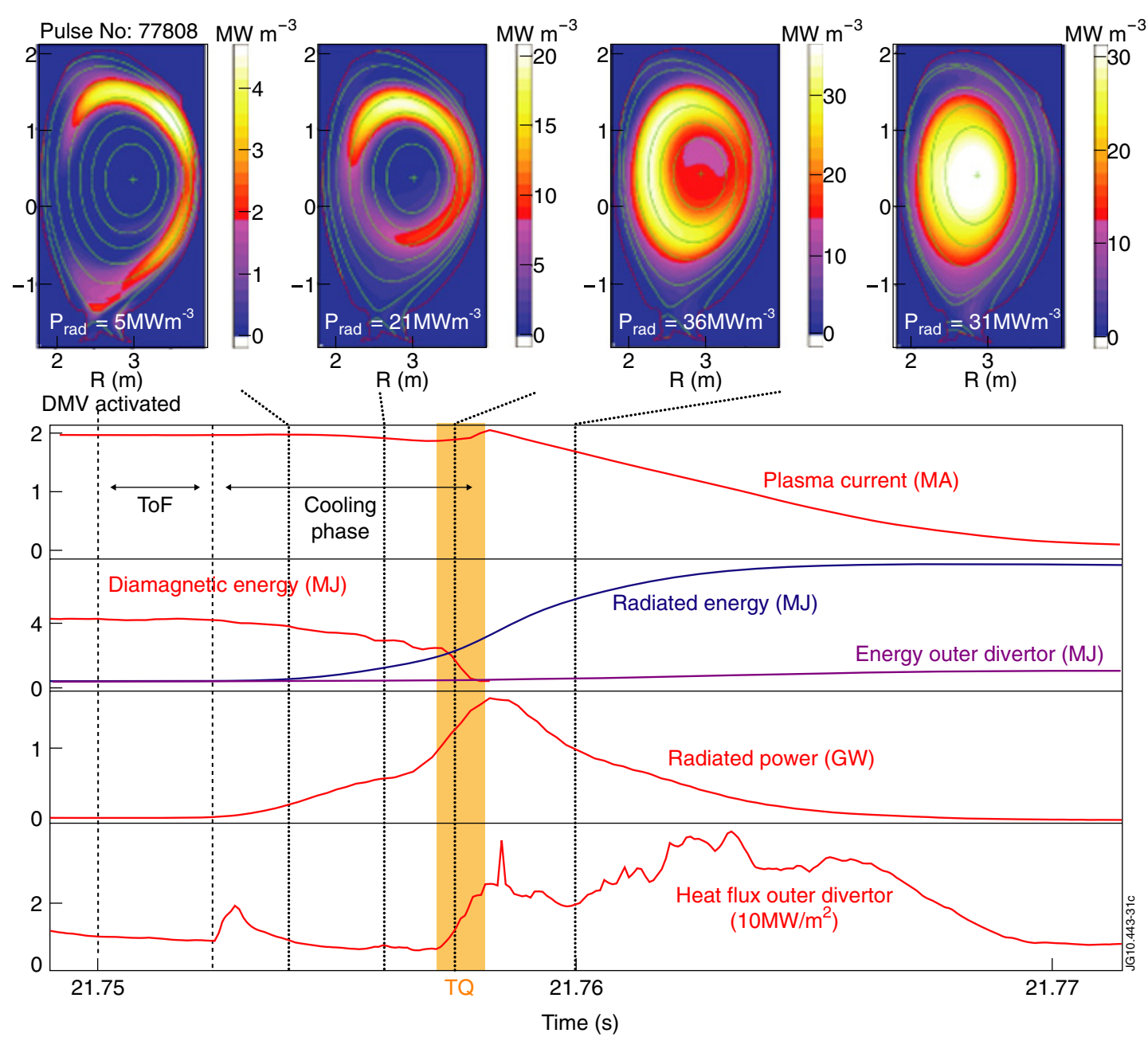

Figure 31. Typical sequence for a disruption initiated by the DMV using an argon/deuterium gas mixture.

has allowed the sequences of events and root causes of the dominant classes of disruptions to be identified [75].

4.3.1. Sideways forces during asymmetric VDEs. During asymmetric VDE disruptions the plasma current and vertical current moment are $n=1$ toroidally asymmetric, leading to sideways forces that in JET can be as high as $4 \mathrm{MN}$ [76]. In most JET disruptions the plasma current asymmetry rotates in the counter-current direction at $\sim 100 \mathrm{~Hz}$, although with large scatter. For ITER, the dynamic amplification of structural forces that would occur if the rotating modes resonated with the vessel at the $8 \mathrm{~Hz}$ fundamental mechanical vessel frequency is a concern. Large plasma current asymmetries $(\sim 10 \%)$ in JET disruptions are, however, observed only for short to moderate current quench (CQ) times (up to 40-60 ms, corresponding to 200-300 ms in ITER if scaled with the plasma cross-section area [77]) and the asymmetries are significantly smaller for longer quench times [78]. This implies that, at the ITER vessel resonance frequency, large asymmetries will only be able to complete a very small number of rotations, limiting the dynamic force amplification.

4.3.2. Disruption mitigation by MGI. Figure 31 shows a typical sequence of events for a disruption initiated by injection of around $2 \times 10^{23}$ particles of an argon/deuterium mixture into a NBI heated plasma using the recently installed disruption mitigation valve (DMV) [74]. After the activation of the DMV, located $4 \mathrm{~m}$ from the plasma, the gas arrives after a flight time of around $2 \mathrm{~ms}$ which initiates the cooling of the plasma edge. When the cold front eventually arrives at a critical flux surface (presumable $q=2$ ) the TQ is triggered, which releases the remaining plasma energy within less than $1 \mathrm{~ms}$, followed by a slower CQ. Using argon/deuterium and neon/deuterium gas mixtures the DMV has proven effective at reducing disruption halo currents, sideways forces, convective heat loads and runaway electron generation. The peak heat loads during the TQ are reduced by the enhanced radiation with MGI. In the cooling phase up to $50 \%$ of the thermal energy stored in the plasma before the DMV is activated is lost, predominantly by radiation, before the TQ. About $40 \%$ of the remaining energy is radiated during the TQ. Thus, only $30 \%$ of the initial energy is lost by convection to PFCs during the TQ, only a small fraction of which is found in the divertor [79]. For VDEs, which have the most peaked heat loads, the peak heat load on the upper dump plate can be reduced from 3.3 to $1.8 \mathrm{MW} \mathrm{m}^{-2}$ when MGI is employed [76]. Halo currents in VDEs can also be reduced by up to $60 \%$ provided the TQ is initiated before a significant vertical movement has taken place. 


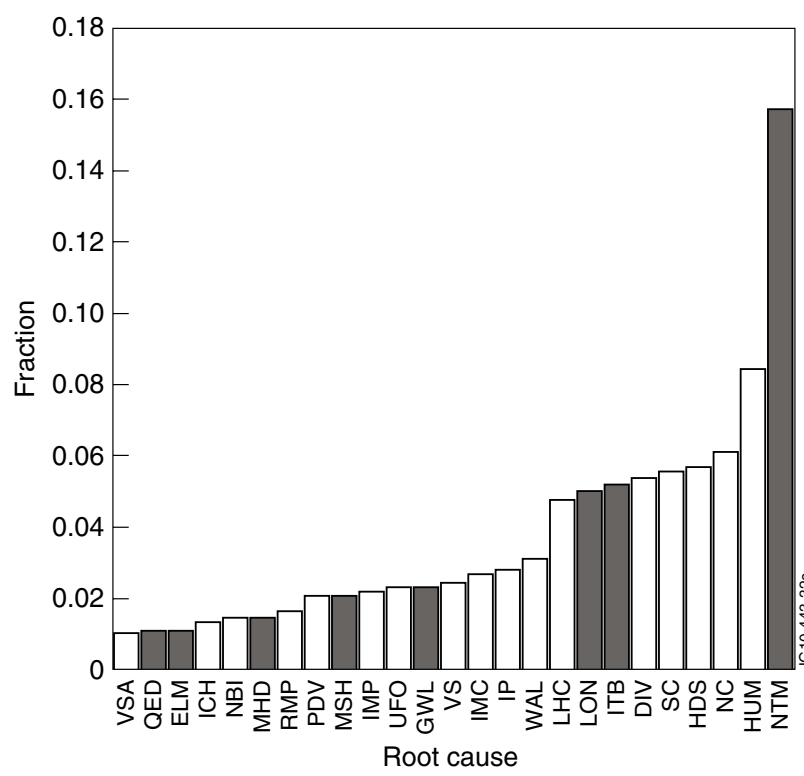

Figure 32. Root causes of JET disruptions. Physics and technical root causes are shown by grey and white coloured boxes, respectively. The dominant cause is found to be NTMs leading to locked modes, followed by human factors (HUM) and density control problems (NC). For a complete list of root causes see [75].

In order to keep forces on PFCs from eddy currents tolerable in ITER, the current decay time must stay above the lower bound of $\tau_{\mathrm{CQ}} / S \approx 1.7 \mathrm{~ms} \mathrm{~m}^{-2}$ (with $S$ the pre-disruption plasma cross-section area). This limit can be reached with pure argon MGI in JET, whereas the deuterium mixtures show a slower current decay. Runaway electron generation is successfully avoided by the injection of argon/deuterium or neon/deuterium mixtures, due to the suppression of the Dreicer mechanism [80]. In contrast, injection of pure argon leads to runaway generation even at low toroidal magnetic fields down to $1.2 \mathrm{~T}$. Although runaway electrons can be safely avoided by MGI in JET disruptions the density reached is still a factor of 50 below the critical density for avalanche suppression [74] which is essential in ITER where runaway currents of up to $10 \mathrm{MA}$ are expected due to the strong avalanche amplification [77, 81]

\subsubsection{Root causes of unintentional disruptions. An} extensive survey of all 2309 JET disruptions with $I_{\mathrm{p}}>1$ MA that occurred from 2000 to 2010 has allowed the sequences of events and root causes of the dominant classes of JET disruptions to be identified [75]. The dominant root cause of disruptions was found to be NTMs that lead to locked modes, followed by human factors and density control problems (figure 32). An important finding is that more than half of all disruptions were caused by reasons other than pure physics instabilities, e.g. subsystem failures $(22 \%)$, control errors $(15.8 \%)$, human errors $(8.3 \%)$ or plasma-wall interactions (7.8\%). Thanks to increased operational experience and improved technical capabilities the global disruptivity has decreased from $\sim 20 \%$ to $3.4 \%$ since the start of JET operations in 1983 . However, about $5 \%$ of all JET disruptions in the last decade were caused by very fast and unpredictable events which may set a lower limit for the JET disruption rate around $0.4 \%$. While it is difficult to extrapolate these results directly to ITER, which will operate in a different operational range and with different technical subsystems, key lessons can still be drawn from the JET experience. Although the initial ITER disruptivity cannot be predicted there will be a learning period before the minimum disruption rate can be achieved, a nontrivial fraction of all disruptions will be caused by technical or human factors and there will likely be a small number of disruptions that can never be predicted.

\section{ITER-relevant ICRH studies}

Three ITER-relevant ICRH systems have been successfully tested on JET [82]; the ITER-like antenna (ILA) [83-86] based on a similar design concept as the ITER ICRH antenna [87] with a closely packed array of short low inductance straps, two of the conventional 'A2' antennas now equipped with external conjugate-T (ECT) matching and two A2 antennas with $3 \mathrm{~dB}$ hybrid couplers. All systems have demonstrated enhanced ELM resilience and have allowed up to 8.6 MW to be coupled on H-mode plasmas with type-I ELMs [18].

Most objectives of the ILA have been demonstrated; matching of an array of closely packed straps, ELM resilience using internal conjugate-T matching, arc detection using Scattering Matrix and Sub-Harmonic Arc Detection (SMAD \& SHAD) systems $[88,89]$ and operation at ITER-relevant power densities (up to $6.2 \mathrm{MW} \mathrm{m}^{-2}$ on L-mode, $4.1 \mathrm{MW} \mathrm{m}^{-2}$ on $\mathrm{H}$-mode) and RF voltages ( $42 \mathrm{kV}$, also on ELMy H-mode plasmas). No evidence of increased impurity production has been found at these power densities which are up to 6 times higher than hitherto achieved on JET [85]. The main issue of concern for ITER was the low coupling $\left(0.8 \Omega \mathrm{m}^{-1}\right)$ measured for the ILA on $\mathrm{H}$-mode plasma with $5 \mathrm{~cm}$ strap to separatrix distance, lower than the originally anticipated $1.5 \Omega \mathrm{m}^{-1}$. To assess the implications of the measured coupling for the coupling predictions made for ITER using RF codes such as TOPICA [90] a strap-separatrix distance scan with welldiagnosed L-mode edge density profiles was carried out and the coupling compared with TOPICA modelling. Good agreement for the effective strap resistance per unit length, $R_{\text {eff }}^{\prime}$, and the effective conductance at the RF probe, $G_{\text {eff }}$, within the error bars was found (figure 33) [86]. This is in agreement with earlier TOPICA validation on Tore Supra [91], DIIID [92] and Alcator C-Mod [90] and, provided the edge density profiles used are realistic, gives confidence in the predictive capability of the code for ITER. A water leak in one of the matching capacitor unfortunately cut the ILA programme short. Whether repairs will be undertaken is still to be decided.

\section{Conclusions and outlook}

Since the last IAEA Conference JET has made significant progress towards the qualification of ITER operating scenarios and the validation of ITER design choices and technologies. The exploitation of the ILW in the coming years will make JET the principal experiment for the development of plasma scenarios compatible with the material combination foreseen for the active phase of ITER. The neutral beam power upgrade will allow stable $\mathrm{H}$-mode operation at higher plasma currents and magnetic fields, allowing access to lower $\rho^{*}$ and $v^{*}$ and higher $\beta_{\mathrm{N}}$ for reduced uncertainties in extrapolations to ITER. 


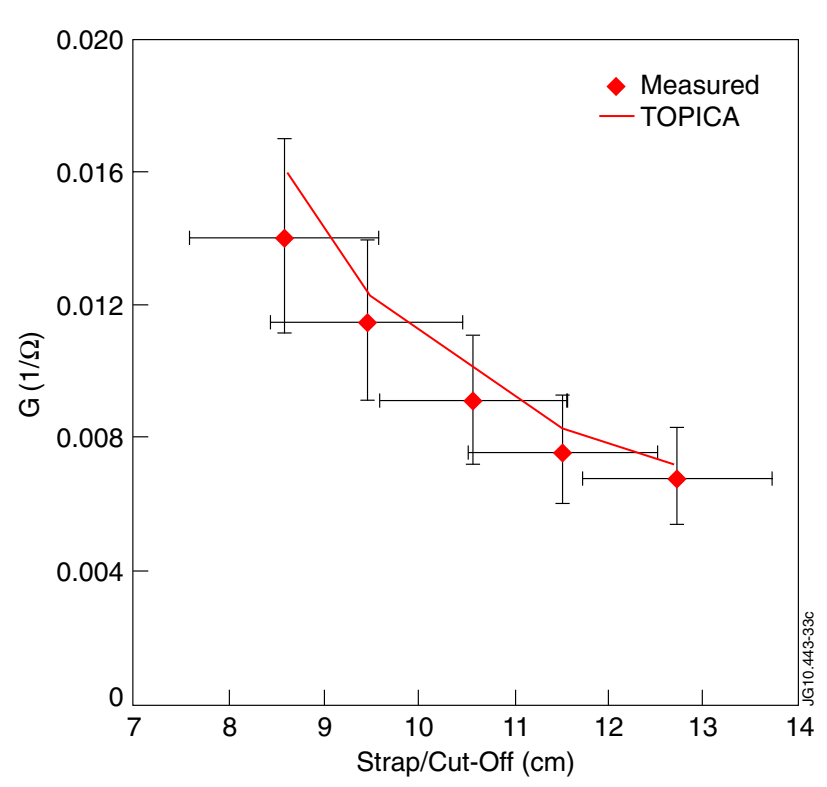

Figure 33. The coupling in terms of effective conductance calculated from TOPICA data. Representative error bars are shown of $\pm 1 \mathrm{~cm}$ on position and $\pm 21 \%$ on power.

The increased pulse length will also be essential to progress the hybrid and steady-state scenarios for ITER. This work is planned to lead up to a full deuterium-tritium campaign in the 2015 time frame for fully integrated tests of the $Q=10$ ITER baseline scenario, including the required active techniques for plasma-wall compatibility (impurity seeding, active ELM mitigation) in a metallic machine.

\section{Acknowledgments}

The results presented in this paper have been obtained by the collective efforts of all JET EFDA contributors, listed in the appendix, and those working under the auspices of the JET Operation Contract. The authors are particularly thankful to M.N.A. Beurskens, S. Brezinsek, E. de la Luna, S. Devaux, P.C. de Vries, F. Durodié, W. Fundamenski, J. Graves, T.C. Hender, E. Joffrin, V.G. Kiptily, P.T. Lang, M. Lehnen, Y. Liang, J. Mailloux, D.C. McDonald, I. Nunes, H. Thomsen, C. Perez von Thun, R. Sartori, A.C.C. Sips and T. Tala. This work was supported by EURATOM and carried out within the framework of the European Fusion Development Agreement. The views and opinions expressed herein do not necessarily reflect those of the European Commission.

\section{(C) Euratom 2011.}

\section{Appendix: List of JET EFDA contributors}

I. Abel $^{1}$, V. Afanesyev ${ }^{2}$, G. Agarici ${ }^{3}$, K.M. Aggarwal ${ }^{4}$, M. Airila ${ }^{5}$, R. Akers ${ }^{6}$, Th. Alarcon ${ }^{3}$, R. Albanese ${ }^{7}$, A. Alexeev ${ }^{8}$, A. Alfier ${ }^{9}$, P. Allan ${ }^{6}$, S. Almaviva ${ }^{10}$, A. Alonso ${ }^{11}$, M. Alonso ${ }^{12}$, B. Alper ${ }^{6}$, H. Altmann ${ }^{6}$, D. Alves ${ }^{12}$, G. Ambrosino ${ }^{7}$, V. Amosov ${ }^{8}$, G. Anda ${ }^{13}$, F. Andersson ${ }^{14}$, E. Andersson Sundén ${ }^{15}$, V. Andreev ${ }^{16}$, Y. Andrew $^{6}$, M. Angelone ${ }^{17}$, M. Anghel ${ }^{18}$, A. Anghel ${ }^{19}$, C. Angioni ${ }^{20}$, G. Apruzzese ${ }^{17}$, N. Arcis ${ }^{6}$, P. Arena ${ }^{21}$,
A. Argouarch ${ }^{3}$, M. Ariola ${ }^{7}$, A. Armitano ${ }^{3}$, R. Armstrong ${ }^{22}$,

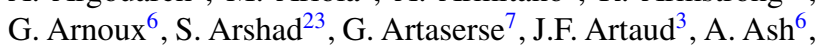
E. Asp ${ }^{24,15}$, O. Asunta ${ }^{25}$, C.V. Atanasiu ${ }^{19}$, G. Atkins ${ }^{6}$, M.D. Axton ${ }^{6}$, C. Ayres $^{6}$, A. Baciero ${ }^{11}$, V. Bailescu ${ }^{26}$, B. Baiocchi ${ }^{27}$, R.A. Baker ${ }^{6}$, I. Balboa ${ }^{6}$, C. Balorin ${ }^{3}$, N. Balshaw ${ }^{6}$, J.W. Banks ${ }^{6}$, Y.F. Baranov ${ }^{6}$, D. Barbier ${ }^{3,24}$, I.L. Barlow ${ }^{6}$, M.A. Barnard ${ }^{6}$, R. Barnsley ${ }^{4}$, L. Barrena ${ }^{11}$, L. Barrera ${ }^{11}$, M. Baruzzo 9 , V. Basiuk ${ }^{3}$, G. Bateman ${ }^{28}$, P. Batistoni ${ }^{17}$, N. Baumgarten ${ }^{29}$, L. Baylor ${ }^{30}$, B. Bazylev ${ }^{31}$, P.S. Beaumont ${ }^{6}$, K. Beausang ${ }^{22}$, M. Bécoulet ${ }^{3}$, N. Bekris ${ }^{31}$, M. Beldishevski ${ }^{6}$, A.C. Bell ${ }^{6}$, F. Belli ${ }^{17}$, M. Bellinger ${ }^{6}$, T. Bellizio ${ }^{7}$, P.S.A. Belo ${ }^{12}$, É. Belonohy ${ }^{20}$, P.E. Bennett ${ }^{6}$, N.A. Benterman ${ }^{6}$, G. Berger-By ${ }^{3}$, H. Bergsåker ${ }^{32}$, H. Berk ${ }^{33}$, J. Bernardo ${ }^{12}$, B. Bertrand ${ }^{3}$, M.N.A. Beurskens ${ }^{6}$, B. Bieg ${ }^{34}$, B. Bienkowska ${ }^{34}$, T. Biewer ${ }^{30}$, T.M. Biewer ${ }^{30}$, M. Bigi ${ }^{9}$, R. Bilato $^{20}$, J. Bird ${ }^{6}$, J. Bizarro ${ }^{12}$, T.R. Blackman ${ }^{6}$, P. Blanchard ${ }^{35,24}$, E. Blanco ${ }^{11}$, J. Blum ${ }^{36}$, V. Bobkov ${ }^{20}$, A. Boboc ${ }^{6}$, D. Boilson ${ }^{22}$, I. Bolshakova ${ }^{37}$, T. Bolzonella ${ }^{9}$, L. Boncagni ${ }^{17}$, G. Bonheure ${ }^{38}$, X. Bonnin ${ }^{3}$, D. Borba ${ }^{39,12}$, A. Borthwick ${ }^{6}$, A. Botrugno ${ }^{17}$, C. Boulbe ${ }^{36}$, F. Bouquey ${ }^{3}$, C. Bourdelle ${ }^{3}$, K.v. Bovert ${ }^{29}$, M. Bowden ${ }^{6}$, T. Boyce ${ }^{6}$, H.J. Boyer ${ }^{6}$, A. Bozhenkov ${ }^{29}$, R.J. Brade ${ }^{6}$, J.M.A. Bradshaw ${ }^{6}$, J. Braet $^{40}$, V. Braic ${ }^{41}$, G.C. Braithwaite ${ }^{6}$, C. Brault ${ }^{3}$, H. Braune ${ }^{42}$, B. Breizman ${ }^{33}$, S. Bremond ${ }^{3}$, P.D. Brennan ${ }^{6}$, A. Brett ${ }^{6}$, J. Breue ${ }^{43}$, S. Brezinsek ${ }^{29}$, M.D.J. Bright ${ }^{6}$, F. Briscoe ${ }^{6}$, M. Brix ${ }^{6}$, M. Brombin 9 , B.C. Brown ${ }^{6}$, D.P.D. Brown ${ }^{6}$, A. Bruschi ${ }^{27}$, J. Brzozowski ${ }^{32}$, J. Bucalossi ${ }^{3}$, M.A. Buckley ${ }^{6}$, T. Budd 6 , R. Budny ${ }^{44}$, R.V. Budny ${ }^{44}$, P. Bunting ${ }^{6}$, P. Buratti ${ }^{17}$, G. Burcea ${ }^{26}$, P.R. Butcher ${ }^{6}$, R.J. Buttery ${ }^{45}$, R. Cação ${ }^{12}$, G. Calabrò ${ }^{17}$, C.P. Callaghan ${ }^{6}$, J.P. Caminade ${ }^{3}$, P.G. Camp ${ }^{6}$, D.C. Campling ${ }^{6}$, J. Canik ${ }^{30}$, B. Cannas $^{46}$, A.J. Capel ${ }^{6}$, G. Carannante ${ }^{7}$, P.J. Card ${ }^{6}$, A. Cardinali ${ }^{17}$, T. Carlstrom ${ }^{45}$, P. Carman ${ }^{6}$, D. Carralero ${ }^{11}$, L. Carraro 9 , T. Carter $^{47}$, B.B. Carvalho ${ }^{12}$, P. Carvalho ${ }^{12}$, A. Casati ${ }^{3}$, C. Castaldo ${ }^{17}$, J. Caughman ${ }^{30}$, R. Cavazzana9, M. Cavinato ${ }^{9}$, M. Cecconello ${ }^{15}$, F.E. Cecil ${ }^{48}$, A. Cenedese ${ }^{9}$, C. Centioli $^{17}$, R. Cesario ${ }^{17}$, C.D. Challis ${ }^{6}$, M. Chandler ${ }^{6}$, C. Chang $^{49}$, A. Chankin ${ }^{20}$, I.T. Chapman ${ }^{6}$, D.J. Child ${ }^{6}$, P. Chiru ${ }^{19}$, G. Chitarin 9 , I. Chugonov ${ }^{2}$, I. Chugunov ${ }^{2}$, D. Ciric $^{6}$, F. Clairet ${ }^{3}$, R.H. Clarke ${ }^{6}$, R. Clay ${ }^{6}$, M. Clever ${ }^{29}$, J.P. $\operatorname{Coad}^{6}$, P.A. Coates ${ }^{6}$, V. Coccorese ${ }^{7}$, V. Cocilovo ${ }^{17}$, S. Coda ${ }^{35}$, R. Coelho ${ }^{12}$, J. Coenen ${ }^{29}$, I. Coffey ${ }^{4}$, L. Colas ${ }^{3}$, M. Cole ${ }^{30}$, S. Collins ${ }^{6}$, S. Combs ${ }^{30}$, J. Compan ${ }^{43}$, J.E. Conboy ${ }^{6}$, S. Conroy ${ }^{15}$, N. Cook $^{6}$, S.P. Cook ${ }^{6}$, D. Coombs ${ }^{6}$, S.R. Cooper ${ }^{6}$, Y. Corre $^{3}$, G. Corrigan ${ }^{6}$, S. Cortes ${ }^{12}$, D. Coster $^{20}$, G.F. Counsell ${ }^{6}$, X. Courtois ${ }^{3}$, M. Cox ${ }^{6}$, T. Craciunescu ${ }^{19}$, S. Cramp ${ }^{6}$, F. Crisanti ${ }^{17}$, O. Croft ${ }^{6}$, K. Crombe $^{50}$, B.J. Crowley ${ }^{6}$, N. Cruz $^{12}$, L. Cupido ${ }^{12}$, M. Curuia ${ }^{18}$, R.A. Cusack ${ }^{6}$, A. Czarnecka ${ }^{34}$, S. Dalley ${ }^{6}$, E.T. Daly ${ }^{6}$, A. Dalziel ${ }^{6}$, D. Darrow ${ }^{44}$, O. David ${ }^{51}$, N. Davies ${ }^{6}$, J.J. Davis ${ }^{6}$, I.E. Day ${ }^{6}$, C. Day ${ }^{31}$, R. De Angelis ${ }^{17}$, G. de Arcas $^{52}$, M.R. de Baar ${ }^{53}$, E. de la Cal ${ }^{11}$, E. de la Luna ${ }^{11,24}$, J.L. de Pablos ${ }^{11}$, G. De Temmerman ${ }^{35}$, G. De Tommasi ${ }^{7}$, P.C. de Vries $^{53}$, R. De-Angelis ${ }^{17}$, F. Degli Agostini ${ }^{9}$, E. Delabie ${ }^{53}$ D. del-Castillo-Negrete ${ }^{30}$ L. Delpech ${ }^{3}$, G. Denisov ${ }^{54}$, A.J. Denyer ${ }^{6}$, R.F. Denyer ${ }^{6}$, S. Devaux ${ }^{20}$, P. Devynck ${ }^{3}$, L. Di Matteo ${ }^{17}$, L. Di Pace ${ }^{17}$, P.J. Dirken ${ }^{6}$,

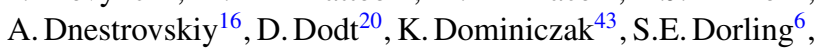
D. Douai ${ }^{3}$, A.P. Down ${ }^{6}$, P.T. Doyle ${ }^{6}$, J.R. Drake ${ }^{32}$, T. Dreischuh ${ }^{55}$, V. Drozdov ${ }^{6}$, P. Dumortier ${ }^{38}$, D. Dunai ${ }^{13}$, 
I. Duran $^{56}$, F. Durodié ${ }^{38}$, K. Dylst ${ }^{40}$, R. Eaton ${ }^{6}$, T. Edlington ${ }^{6}$, A.M. Edwards ${ }^{6}$, D.T. Edwards ${ }^{6}$, P.K. Edwards ${ }^{6}$, Th. Eich ${ }^{20}$, A. Ekedahl ${ }^{3}$, T. Elevant ${ }^{32}$, A. Elfimov ${ }^{12}$, B. Ellingboe ${ }^{22}$, C.G. Elsmore ${ }^{6}$, B. Emmoth ${ }^{57}$, G. Ericsson ${ }^{15}$, L.G. Eriksson ${ }^{58}$, A. Eriksson ${ }^{14}$, B. Esposito ${ }^{17}$, H.G. Esser ${ }^{29}$, T. Estrada ${ }^{11}$, E.A. Evangelidis ${ }^{59}$, G.E. Evans ${ }^{6}$, G.D. Ewart ${ }^{6}$, D.T. Ewers ${ }^{6}$, G. Falchetto ${ }^{3}$, D. Falie ${ }^{19}$, J.G.A. Fanthome ${ }^{6}$, D. Farina ${ }^{27}$, J.W. Farthing ${ }^{6}$, A. Fasoli ${ }^{35}$, B. Faugeras ${ }^{36}$, N. Fedorczak ${ }^{3}$, R.C. Felton ${ }^{6}$, C. Fenzi $^{3}$, H. Fernandes ${ }^{12}$, J.A. Ferreira ${ }^{11}$, J. Ferreira ${ }^{12}$, J. Ferron ${ }^{45}$, J.A. Fessey ${ }^{6}$, L. Figini ${ }^{27}$, A. Figueiredo $^{12}$, J. Figueiredo ${ }^{12}$, P. Finburg ${ }^{6}$, K.H. Finken ${ }^{29}$, U. Fischer ${ }^{31}$, N. Fitzgerald ${ }^{22}$, J. Flanagan ${ }^{6}$, C. Fleming ${ }^{6}$, A. Fonseca ${ }^{12}$, A.D. Forbes ${ }^{6}$, O. Ford ${ }^{1}$, A. Formisano ${ }^{7}$, D. Fraboulet ${ }^{3}$, R.J. Francis ${ }^{6}$, L. Frassinetti ${ }^{32}$, R. Fresa ${ }^{7}$, J.P. Friconneau ${ }^{51}$, D. Frigione ${ }^{17}$, J.C. Fuchs ${ }^{20}$, K. Fullard ${ }^{6}$, W. Fundamenski ${ }^{6}$, M. Furno Palumbo ${ }^{7}$, J. Gafert ${ }^{20}$, K. Gál ${ }^{13}$, R. Galvão ${ }^{12}$, S. Garavaglia ${ }^{27}$, X. Garbet ${ }^{3}$, J. Garcia ${ }^{3}$, M. Garcia Munoz $^{20}$, W. Gardner ${ }^{30}$, P. Garibaldi ${ }^{3}$, D. Garnier ${ }^{3}$, L. Garzotti ${ }^{6}$, M. Gatu Johnson ${ }^{15}$, P. Gaudio ${ }^{10}$, E. Gauthier ${ }^{3}$, J.W. Gaze ${ }^{6}$, D.F. Gear ${ }^{6}$, J. Gedney ${ }^{6}$, S.J. Gee ${ }^{6}$, M. Gelfusa ${ }^{10}$, E. Genangeli ${ }^{24,17}$, S. Gerasimov ${ }^{6}$, A. Geraud ${ }^{3}$, T. Gerbaud $^{3}$, M. Gherendi ${ }^{19}$, N. Ghirelli ${ }^{3}$, J.C. Giacalone ${ }^{3}$, L. Giacomelli60, C.S. Gibson ${ }^{6}$, C. Gil ${ }^{3}$, S.J. Gilligan ${ }^{6}$, C.G. Gimblett ${ }^{6}$, D. Gin ${ }^{2}$, E. Giovannozzi ${ }^{17}$, C. Giroud ${ }^{6}$, G. Giruzzi ${ }^{3}$, S. Glowacz ${ }^{34}$, J. Godwin ${ }^{6}$, J.K. Goff ${ }^{6}$, P. Gohil ${ }^{45}$, V. Goloborod'ko ${ }^{61}$, B. Gonçalves ${ }^{12}$, M. Goniche ${ }^{3}$, S. Gonzales ${ }^{11}$, S.M. González de Vicente ${ }^{40}$, A. Goodyear ${ }^{6}$, N. Gorelenkov ${ }^{44}$, G. Gorini ${ }^{27}$, R. Goulding ${ }^{30}$, B. Graham ${ }^{6}$, D. Graham ${ }^{6}$, M.E. Graham ${ }^{6}$, G. Granucci ${ }^{27}$, J. Graves ${ }^{35}$, N.R. Green ${ }^{6}$, H. Greuner ${ }^{20}$, E. Grigore ${ }^{19}$, F.S. Griph ${ }^{6}$, C. Grisolia ${ }^{3}$, G. Gros ${ }^{3}$, G. Grossetti ${ }^{27}$, M. Groth ${ }^{25}$, S. Grünhagen ${ }^{6}$, M.P. Gryaznevich ${ }^{6}$, R. Guirlet ${ }^{3}$, B. Gulejova ${ }^{35}$, J. Gunn $^{3}$, A. Gupta ${ }^{29}$, P. Guzdar ${ }^{62}$, P. Hacek ${ }^{56}$, L.J. Hackett ${ }^{6}$, S. Hacquin ${ }^{3}$, B. Haist ${ }^{6}$, A. Hakola ${ }^{25}$, S.J. Hall ${ }^{6}$, S.P. Hallworth Cook $^{6}$, D.T. Hamilton ${ }^{6}$, H. $\mathrm{Han}^{63}$, R.C. Handley ${ }^{6}$, S. Harding ${ }^{6}$, J.D.W. Harling ${ }^{6}$, D. Harting ${ }^{29}$, M.J. Harvey ${ }^{6}$, T.D.V. Haupt ${ }^{6}$, E. Havlickova ${ }^{56}$, N.C. Hawkes ${ }^{6}$, R. Hawryluk ${ }^{44}$, J.H. Hay ${ }^{6}$, N. Hayashi ${ }^{49}$, P.W. Haydon ${ }^{6}$, I.R. Hayward ${ }^{6}$, S. Hazel ${ }^{6}$, P.J.L. Heesterman ${ }^{6}$, W. Heidbrink ${ }^{44}$, J. Heikkinen ${ }^{5}$, C. Hellesen ${ }^{15}$, T. Hellsten ${ }^{32}$, O.N. Hemming ${ }^{6}$, T.C. Hender ${ }^{6}$, M. Henderson ${ }^{64}$, C. Hennig ${ }^{20}$, V. Hennion ${ }^{3}$, C. Hidalgo ${ }^{11}$, S. Higashijima ${ }^{49}$, J.W. Hill ${ }^{6}$, M. Hill ${ }^{6}$, K. Hill ${ }^{44}$, J. Hillairet ${ }^{3}$, D. Hillis ${ }^{30}$, T. Hirai ${ }^{43}$, M. Hitchin 6 , J. Hobirk ${ }^{20}$, C. Hogan ${ }^{30}$, C.H.A. Hogben ${ }^{6}$, G.M.D. Hogeweij ${ }^{53}$, I.C. Hollingham ${ }^{6}$, R. Holyaka ${ }^{37}$, D.A. Homfray ${ }^{6}$, G. Honeyands ${ }^{6}$, S.H. Hong ${ }^{3}$, C. Hopf ${ }^{20}$, B.A. Horn ${ }^{6}$, A.R. Horton 6 , L.D. Horton ${ }^{24,58}$, S.P. Hotchin ${ }^{6}$, M.R. Hough ${ }^{6}$, W. Houlberg ${ }^{30}$, D.F. Howell ${ }^{6}$, M. Hron ${ }^{56}$, A. Huber $^{29}$, T.M. Huddleston ${ }^{6}$, Z. Hudson ${ }^{6}$, M. Hughes ${ }^{6}$, M. Hühnerbein ${ }^{43}$, C.C. Hume ${ }^{6}$, A.J. Hunt ${ }^{6}$, C.L. Hunter ${ }^{6}$, T.S. Hutchinson ${ }^{6}$, S. Huygen ${ }^{38}$, G. Huysmans ${ }^{3}$, V. Hynönen ${ }^{25}$,

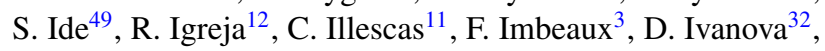
E. Ivings ${ }^{6}$, S. Jachmich ${ }^{38}$, G. Jackson ${ }^{45}$, P. Jacquet ${ }^{6}$, K. Jakubowska ${ }^{34}$, M. Jakubowski ${ }^{42}$, P.V. James ${ }^{6}$, R.J.E. Jaspers ${ }^{53}$, S. Jednorog ${ }^{34}$, I. Jenkins ${ }^{6}$, M.A.C. Jennison ${ }^{6}$, C. Jeskins ${ }^{6}$, O. Jin Kwon ${ }^{65}$, E. Joffrin ${ }^{3,24}$, M.F. Johnson ${ }^{6}$, R. Johnson ${ }^{6}$, T. Johnson ${ }^{32}$, D. Jolovic ${ }^{29}$, V. Jonauskas ${ }^{66}$, E.M. Jones ${ }^{6}$, G. Jones ${ }^{6}$, H.D. Jones ${ }^{6}$, T.T.C. Jones ${ }^{6}$, M. Jouvet ${ }^{3}$, C. Jupén ${ }^{67}$, I. Kachtchouk ${ }^{8}$, J. Kaczmarczyk ${ }^{34}$, A. Kallenbach ${ }^{20}$, J. Källne ${ }^{68}$, D. Kalupin ${ }^{29}$, S. Kálvin ${ }^{13}$, G. Kamelander ${ }^{61}$, R. Kamendje ${ }^{69}$ A. Kappatou ${ }^{70}$,
S. Karttunen ${ }^{5}$, W. Kasparek ${ }^{71}$, I. Katramados ${ }^{6}$, M. Kaufmann ${ }^{20}$, G. Kaveney ${ }^{6}$, A.S. Kaye ${ }^{6}$, M.J. Kear ${ }^{6}$, D.L. Keeling, D. Kelliher ${ }^{22}$, M. Kempenaars ${ }^{6}$, P. Khilar ${ }^{6}$, N.G. Kidd ${ }^{6}$, M. Kiisk ${ }^{72}$, K.M. Kim ${ }^{63}$, R.F. King ${ }^{6}$, D.J. Kinna ${ }^{6}$, V. Kiptily ${ }^{6}$, G. Kirnev ${ }^{16}$, N. Kirneva ${ }^{16}$, K. Kirov ${ }^{6}$, A. Kirschner ${ }^{29}$, R. Kisielius ${ }^{66}$, D. Kislov ${ }^{16}$, G. Kiss ${ }^{29}$, T. Kiviniemi ${ }^{25}$, G. Kizane ${ }^{73}$, A. Klein ${ }^{74}$, A. Klix ${ }^{31}$, M. Knaup ${ }^{29}$, K. Kneuper ${ }^{6}$, H. Kneupner ${ }^{29}$, P.J. Knight ${ }^{6}$, S.J. Knipe ${ }^{6}$, M. Kocan ${ }^{3}$, F. Köchl ${ }^{61}$, G. Kocsis ${ }^{13}$, C. Konz ${ }^{20}$, T. Koppitz ${ }^{43}$, A. Korotkov ${ }^{6}$, H.R. Koslowski ${ }^{29}$, V. Kotov ${ }^{29}$, M.D. Kovari ${ }^{6}$, K. Kovarik ${ }^{56}$, G. $\mathrm{Kramer}^{44}$, A. Krasilnikov ${ }^{8}$, V. Krasilnikov ${ }^{8}$, S. Kraus ${ }^{29}$, A. $\mathrm{Kreter}^{29}$, K. Krieger ${ }^{20}$, A. Kritz ${ }^{28}$, Y. Krivchenkov ${ }^{6}$, U. Kruezi ${ }^{29}$, M. Krychowiak ${ }^{42}$, S. Krylov ${ }^{16}$, I. Ksiazek ${ }^{34}$, M. Kubic ${ }^{56}$, S. Kuhn ${ }^{61}$, W. Kühnlein ${ }^{43}$, T. Kurki-Suonio ${ }^{25}$, A. Kurowski ${ }^{34}$, B. Kuteev ${ }^{16}$, A. Kuyanov ${ }^{16}$, R. La Haye ${ }^{45}$, M. Laan ${ }^{72}$, C. Labate ${ }^{7}$, A. Lachichi ${ }^{6}$, N. Lam ${ }^{6}$, P. Lang ${ }^{20}$, M.T. Large ${ }^{6}$, I. Lassiwe ${ }^{29}$, J.R. Last ${ }^{6}$, K.D. Lawson ${ }^{6}$, M. Laxåback ${ }^{24,32}$, R.A. Layne ${ }^{6}$, E. Lazzaro ${ }^{27}$, F. Le Guern $^{3}$, B. LeBlanc ${ }^{44}$, H.J. Leggate ${ }^{22}$, M. Lehnen ${ }^{29}$, M. Leigheb ${ }^{17}$, I. Lengar ${ }^{75}$, M. Lennholm ${ }^{24,58}$, E. Lerche ${ }^{38}$, C.N. Lescure ${ }^{6}$, Y. Li $^{74}$, A. Li Puma ${ }^{3}$, Y. Liang ${ }^{29}$, J. Likonen ${ }^{5}$, Y. Lin $^{74}$, J. Linke ${ }^{43}$, S.A. Linstead ${ }^{6}$, B. Lipshultz ${ }^{74}$, X. Litaudon ${ }^{3}$, A.G. Litvak ${ }^{54}$, Y. Liu ${ }^{6}$, T. Loarer ${ }^{3}$, A. Loarte ${ }^{64}$, R.C. Lobel $^{6}$, P.J. Lomas $^{6}$, F.D. Long 6 , J. Lönnroth ${ }^{25}$, D.J. Looker ${ }^{6}$, J. Lopez ${ }^{11}$, Ph. Lotte ${ }^{3}$, M.J. Loughlin ${ }^{6}$, A.B. Loving ${ }^{6}$, C. Lowry ${ }^{24,58}$, T. Luce ${ }^{45}$, R.M.A. Lucock ${ }^{6}$, A. Lukanitsa ${ }^{76}$, A.M. Lungu ${ }^{19}$, C.P. Lungu ${ }^{19}$, A. Lyssoivan ${ }^{38}$, P. Macheta ${ }^{6}$, A.S. Mackenzie ${ }^{6}$, M. Macrae ${ }^{6}$, G. Maddaluno ${ }^{17}$, G.P. Maddison ${ }^{6}$, J. Madsen ${ }^{77}$, P. Maget $^{3}$, C. Maggi ${ }^{20}$, H. Maier $^{20}$, J. Mailloux ${ }^{6}$, M. Makowski ${ }^{45}$, C.J. Manning ${ }^{6}$, M. Mansfield ${ }^{22}$, M.E. Manso ${ }^{12}$, P. Mantica ${ }^{27}$, M. Mantsinen ${ }^{25}$, M. $\operatorname{Maraschek}^{20}$, C. Marchetto ${ }^{27}$ M.A. Marchitti ${ }^{\text {, }}$ M. Mardenfeld ${ }^{44}$, J.L. Marechal $^{3}$, M. Marinelli ${ }^{10}$, A. Marinoni ${ }^{35}$, M. Marinucci ${ }^{17}$, J. Märki ${ }^{35}$, D. Marocco ${ }^{17}$, C.A. Marren ${ }^{6}$, D. $\operatorname{Martin}^{6}$, D.L. Martin ${ }^{6}$, G. Martin ${ }^{3}$, Y. Martin ${ }^{35}$, J.R. Martín-Solís ${ }^{78}$, K. Masaki ${ }^{49}$, A. Masiello ${ }^{9}$, M. Maslov ${ }^{35}$, C. Maszl ${ }^{61}$, A. Matilal ${ }^{6}$, M. Mattei ${ }^{7}$, G.F. Matthews ${ }^{6}$, F. Maviglia ${ }^{7}$, C.R. May $^{6}$, M. Mayer ${ }^{20}$, M.L. Mayoral ${ }^{6}$, D. Mazon ${ }^{3}$, C. Mazzotta ${ }^{17}$, E. Mazzucato ${ }^{44}$, P. $\mathrm{McCarthy}^{22}$, K.G. McClements ${ }^{6}$, K. McCormick ${ }^{20}$, P.A. McCullen ${ }^{6}$ D. McCune ${ }^{44}$, D.C. McDonald ${ }^{6}$, R. Mcgregor ${ }^{6}$, J.P. Mckivitt ${ }^{6}$, A. Meakins ${ }^{6}$, F. Medina ${ }^{11}$, A.G. Meigs 6 , M. Menard ${ }^{45}$, L. Meneses ${ }^{12}$, S. Menmuir ${ }^{79}$, I.R. Merrigan ${ }^{6}$, Ph. Mertens ${ }^{29}$, A. Messiaen ${ }^{38}$, H. Meyer ${ }^{6}$, G. Miano ${ }^{7}$, M. Miele ${ }^{7}$, P. Migliucci ${ }^{10}$, A.G. Miller ${ }^{6}$, S.F. Mills ${ }^{6}$, J.J. Milnes ${ }^{6}$, K. Min Kim ${ }^{63}$, T. Mindham ${ }^{6}$, F. Mirizzi ${ }^{17}$, E. Mirones ${ }^{11}$, M. Mironov ${ }^{2}$, R. Mitteau ${ }^{3}$, J. Mlynar ${ }^{56}$, P. Mollard ${ }^{3}$, I. Monakhov ${ }^{6}$, P. Monier-Garbet ${ }^{3}$, R. Mooney ${ }^{6}$, S. Moradi ${ }^{80}$, D. Moreau ${ }^{3}$, Ph. Moreau ${ }^{3}$, L. Moreira ${ }^{6}$, A. Morgan ${ }^{6}$, P.D. Morgan ${ }^{6}$, C. Morlock ${ }^{24,29}$, A. $\operatorname{Moro}^{27}$, A.W. Morris ${ }^{6}$, G.L. Mort ${ }^{6}$, C. Mrozek ${ }^{20}$, A. Mueck ${ }^{35}$, H.W. Müller ${ }^{20}$, M. Murakami ${ }^{30}$, A. Murari ${ }^{24,9}$, I. Mustata ${ }^{19}$, F. Nabais ${ }^{12}$, E. Nardon ${ }^{3}$, G. Nash ${ }^{6}$, V. Naulin ${ }^{77}$, M.F.F. Nave ${ }^{12}$, R. Nazikian ${ }^{44}$, I. Nedzelski ${ }^{12}$, C.R. Negus ${ }^{6}$, J.D. Neilson ${ }^{6}$ A. Neto ${ }^{12}$, R. $\mathrm{Neu}^{20}$, O. Neubauer ${ }^{29}$, G.J. Newbert ${ }^{6}$, M. Newman ${ }^{6}$, K.J. Nicholls ${ }^{6}$, A. Nicolai ${ }^{29}$, L. Nicolas ${ }^{3}$, P. Nieckchen ${ }^{24,20}$, P. Nielsen ${ }^{9}$, A.H. Nielsen ${ }^{77}$, S.K. Nielsen ${ }^{77}$, G. Nielson ${ }^{44}$, J. Nieto ${ }^{52}$, M.P.S. Nightingale ${ }^{6}$, C. Noble ${ }^{6}$, M. Nocente ${ }^{27}$, M. Nora ${ }^{25}$, H. Nordman ${ }^{14}$, M. Norman ${ }^{6}$, J-M. Noterdaeme ${ }^{20}$, S. Nowak ${ }^{27}$, I. Nunes ${ }^{24,12}$, 
F. Ognissanto ${ }^{27}$, T. O'Gorman ${ }^{22}$, S. Olariu ${ }^{81}$, A. Oleynikov ${ }^{8}$, M. O'Mullane ${ }^{82}$, J. Ongena ${ }^{38}$, F. Orsitto ${ }^{17}$, O.I. Oswuigwe ${ }^{6}$, M. Ottaviani ${ }^{3}$, N. Oyama ${ }^{49}$, D. Pacella ${ }^{17}$, K. Paget ${ }^{6}$, S. Palazzo ${ }^{21}$, J. Pamela ${ }^{3}$, S. Pamela ${ }^{3}$, R. Panek ${ }^{56}$, L. Pangione ${ }^{6}$, A. Panin ${ }^{29}$, Th. Panis ${ }^{35}$, A. Pankin ${ }^{28}$, A. Pantea ${ }^{19}$, V. Parail ${ }^{6}$, Th. Parisot ${ }^{3}$, A. Parkin 6 , A. Parsloe ${ }^{6}$, B.T. Parsons ${ }^{6}$, R. Pasqualotto ${ }^{9}$, P. Pastor $^{3}$, R. Paterson ${ }^{6}$, M.K. Paul ${ }^{29}$, D. Peach ${ }^{6}$, R.J.H. Pearce ${ }^{6}$, B.J. Pearson ${ }^{6}$, I.J. Pearson ${ }^{6}$, L.C. Pedrick ${ }^{6}$, M.A. Pedrosa ${ }^{11}$, B. Pegourie ${ }^{3}$, R. Pereira ${ }^{12}$, E. Perelli $\mathrm{Cippo}^{27}$, G. Pereverzev ${ }^{20}$, A. Perevezentsev ${ }^{6}$, Ch. Perez von Thun ${ }^{24,} 20$, V. Pericoli-Ridolfini ${ }^{39,} 17$, A. Perona ${ }^{83}$ Y. Perrot $^{51}$, S. Peruzzo ${ }^{9}$, S. Peschanyy ${ }^{31}$, G. Petravich ${ }^{13}$, L. Petrizzi $^{17}$, V. Petrov ${ }^{8}$, V. Petrzilka ${ }^{56}$, V. Philipps ${ }^{29}$, G. Piazza $^{31}$, F. Piccolo ${ }^{6}$, A. Pietropaolo ${ }^{27}$, M. Pillon ${ }^{17}$, S.D. Pinches ${ }^{6}$, T. Pinna ${ }^{17}$, G. Pintsuk ${ }^{43}$, P. Piovesan ${ }^{9}$, A. Pironti ${ }^{7}$, F. Pisano ${ }^{46}$, R. Pitts ${ }^{64}$, B. Plaum ${ }^{71}$, V. Plyusnin ${ }^{12}$, M. Polasik ${ }^{34}$, F.M. Poli ${ }^{84}$, N. Pomaro' , O. Pompilian ${ }^{19}$, L. Poncet $^{3}$, P.J. Pool ${ }^{6}$, S. Popovichev ${ }^{6}$, F. Porcelli ${ }^{83}$, M.T. Porfiri ${ }^{17}$, C. Portafaix ${ }^{3}$, A. Pospieszczyk ${ }^{29}$, G. Possnert ${ }^{68}$, G. Prestopino ${ }^{10}$, P. Prior ${ }^{6}$, R. Prokopowicz ${ }^{34}$, I. Proverbio ${ }^{27}$, R. Pugno ${ }^{20}$, M.E. Puiatti ${ }^{9}$, K. Purahoo ${ }^{6}$, V. Pustovitov ${ }^{16}$, Th. Pütterich ${ }^{20}$, D. Püttmann-Kneupner ${ }^{29}$, A. Quercia ${ }^{7}$, E. Rachlew ${ }^{79}$, R. Rademaker ${ }^{53}{ }^{24}$, T. Rafiq ${ }^{28}$, M.S.J. Rainford ${ }^{6}$, G. Ramogida ${ }^{17}$, K. Rantamäki ${ }^{5}$, J. Rapp ${ }^{29}$, J.J. Rasmussen ${ }^{77}$, G. Rattá ${ }^{11}$, G. Ravera ${ }^{17}$, M. Reich ${ }^{20}$, R. Reichle ${ }^{3}$, D. Reiser ${ }^{29}$, R. Reiss ${ }^{3}$, D. Reiter ${ }^{29}$, D. Rendell ${ }^{6}$, C. Reux $^{3}$, G. Rewoldt ${ }^{74}$, T.T. Ribeiro ${ }^{12}$, V. Riccardo ${ }^{6}$, D. Richards ${ }^{6}$, F. Rigollet ${ }^{3}$, F.G. Rimini ${ }^{24,58}$, L. $\operatorname{Rios}^{11}$, M. Riva ${ }^{17}$, J.E.C. Roberts ${ }^{6}$, R.J. Robins ${ }^{6}$, D.S. Robinson ${ }^{6}$, S.A. Robinson ${ }^{6}$, D.W. Robson ${ }^{6}$, H. Roche ${ }^{3}$, M. Rödig ${ }^{43}$, N. Rodionov ${ }^{8}$, V. Rohde ${ }^{20}$, A. Rolfe ${ }^{6}$, M. Romanelli ${ }^{6}$, F. Romanelli ${ }^{24,17}$, A. Romano ${ }^{17}$, J. Romero ${ }^{11}$, E. Ronchi ${ }^{15}$, S. Rosanvallon ${ }^{3}$, Ch. Roux ${ }^{3}$, S. Rowe ${ }^{6}$, M. Rubel $^{32}$, G. Rubinacci ${ }^{7}$, L. Ruchko ${ }^{12}$, M. Ruiz $^{52}$, C. Ruset ${ }^{19}$, M. Russell ${ }^{6}$, A. Ruth ${ }^{22}$, L. Ryc ${ }^{34}$, A. Rydzy ${ }^{17}$, F. Ryter $^{20}$, J. Rzadkiewicz ${ }^{34}$, S. Saarelma ${ }^{6}$, F. Sabathier ${ }^{3}$, R. Sabot ${ }^{3}$, S. Sadakov ${ }^{29}$, P. Sagar ${ }^{6}$, G. Saibene ${ }^{23}$, A. Saille ${ }^{3}$, F. Saint-Laurent ${ }^{3}$, A. Salmi ${ }^{25}$, R. Salomaa ${ }^{25}$, F. Salzedas ${ }^{12}$, U. Samm $^{29}$, P. Sanchez ${ }^{11}$, S. Sanders ${ }^{6}$, S.G. Sanders ${ }^{6}$, G. Sandford ${ }^{6}$, K. Sandland ${ }^{6}$, P. Sandquist ${ }^{14}$, D.E.G. Sands ${ }^{6}$, M.I.K. Santala ${ }^{25}$, F. Sartori ${ }^{23}$, R. Sartori ${ }^{23}$, O. Sauter ${ }^{35}$, A. Savelyev $^{2}$, A. Savtchkov ${ }^{29}$, S.C. Scales ${ }^{6}$, A. Scarabosio ${ }^{20}$, N. Schaefer ${ }^{3}$, Ch. Schlatter ${ }^{35}$, V. Schmidt ${ }^{9}$, A. Schmidt ${ }^{29}$, O. Schmitz $^{29}$, S. Schmuck ${ }^{42}$, M. Schneider ${ }^{3}$, M. Scholz ${ }^{34}$, K. Schöpf ${ }^{61}$, B. Schweer ${ }^{29}$, J. Schweinzer ${ }^{20}$, B. Scott ${ }^{20}$, M. Seki ${ }^{49}$, L. Semeraro ${ }^{17}$, A. Semerok ${ }^{85}$, G. Sergienko ${ }^{29}$, F. Serra ${ }^{12}$, M. Sertoli ${ }^{20}$, M.M.J. Shannon ${ }^{6}$, S.E. Sharapov ${ }^{6}$, S.R. Shaw ${ }^{6}$, A. Shevelev ${ }^{2}$, R. Sievering ${ }^{43}$, C.A. Silva ${ }^{12}$, P.A. Simmons ${ }^{6}$, A. Simonetto ${ }^{27}$, D. Simpson ${ }^{6}$, S. Sipila ${ }^{25}$, A.C.C. $\operatorname{Sips}^{24,58}$, A. Sirinelli ${ }^{6}$, H. Sjöstrand ${ }^{15}$, D. Skopintsev ${ }^{8}$, K. Slabkowska. ${ }^{34}$, P.G. Smith ${ }^{6}$, J. Snipes ${ }^{74}$, L. Snoj ${ }^{75}$, S. Snyder $^{28}$, S. Soare ${ }^{18}$, E.R. Solano ${ }^{11}$, S. Soldatov ${ }^{38}$, A. Soleto ${ }^{11}$, W. Solomon ${ }^{44}$, C. Soltane ${ }^{3,24}$, P. Sonato ${ }^{9}$, A. Sopplesa ${ }^{9}$, A. Sorrentino ${ }^{7}$, J. Sousa ${ }^{12}$, C.B.C. Sowden ${ }^{6}$, C. Sozzi ${ }^{27}$, P. Späh ${ }^{31}$, T. Spelzini ${ }^{6}$, J. Spence ${ }^{6}$, F. Spineanu ${ }^{19}$, P. Spuig ${ }^{3}$, A. Stäbler ${ }^{20}$, R.D. Stagg ${ }^{6}$, M.F. Stamp ${ }^{6}$, V. Stancalie ${ }^{19}$, P. Stangeby ${ }^{45}$, C. Stan-Sion ${ }^{81}$, D.E. Starkey ${ }^{6}$, M.J. Stead ${ }^{6}$, A.V. Stephen ${ }^{6}$, A.L. Stevens ${ }^{6}$, J. Stober ${ }^{20}$, R.B. Stokes ${ }^{6}$, D. Stork ${ }^{6}$, D. Stoyanov ${ }^{55}$, J. Strachan ${ }^{44}$, P. Strand ${ }^{14}$, M. Stransky ${ }^{14}$, D. Strauss ${ }^{31}$, D. Strintzi ${ }^{70}$, W. Studholme ${ }^{6}$, Y. Su Na ${ }^{63}$, F. Subba ${ }^{83}$, H.P. Summers ${ }^{82}$,
Y. Sun $^{29}$, C. Surdu-Bob ${ }^{19}$, E. Surrey ${ }^{6}$, D.J. Sutton ${ }^{6}$, J. Svensson ${ }^{20}$, D. Swain $^{30}$, B.D. Syme ${ }^{6}$, I.D. Symonds ${ }^{6}$, T. Szepesi ${ }^{13}$, A. Szydlowski ${ }^{34}$, F. Tabares ${ }^{11}$, V. Takalo ${ }^{86}$, H. Takenaga ${ }^{49}$, T. Tala ${ }^{5}$, A.R. Talbot ${ }^{6}$, C. Taliercio 9 ,

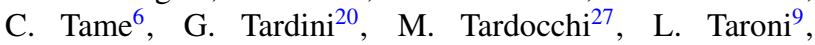
G. Telesca ${ }^{38}$, A. Terra ${ }^{29}$, A.O. Terrington ${ }^{6}$, D. Testa ${ }^{35}$, J.M. Theis ${ }^{3}$, J.D. Thomas ${ }^{6}$, P.D. Thomas ${ }^{6}$, P.R. Thomas ${ }^{23}$, V.K. Thompson ${ }^{6}, \quad$ H. Thomsen ${ }^{20}$, C. Thomser ${ }^{29}$, A. Thyagaraja ${ }^{6}$, P.A. Tigwell ${ }^{6}$, I. Tiseanu ${ }^{19}$, R. Tivey ${ }^{24,58}$, J.M. $\operatorname{Todd}^{6}$, T.N. $\operatorname{Todd}^{6}$, M.Z. $\operatorname{Tokar}^{29}$, S. Tosti ${ }^{17}$, P. Trabuc ${ }^{3}$, J.M. Travere ${ }^{3}$, W. Treutterer ${ }^{20}$, P. Trimble ${ }^{6}$, A. $\operatorname{Trkov}^{75}$, E. Trukhina ${ }^{16}$, M. Tsalas ${ }^{24,59}$, H. TsigeTamirat $^{31}$, E. Tsitrone ${ }^{3}$, D. Tskhakaya jun ${ }^{61}$, O. Tudisco ${ }^{17}$, S. Tugarinov ${ }^{8}$, M.M. Turner ${ }^{22}$, G. Turri ${ }^{35}$, S.G.J. Tyrrell ${ }^{6}$, N. Umeda ${ }^{49}$, B. Unterberg ${ }^{29}$, H. Urano ${ }^{49}$, A.J. Urquhart ${ }^{6}$, I. Uytdenhouwen ${ }^{40}$, A. Vaccaro $^{31}$, A.P. Vadgama ${ }^{6}$, G. Vagliasindi ${ }^{17}$, D. Valcarcel ${ }^{12}$, M. Valisa ${ }^{9}$, J. Vallory ${ }^{3}$, M. Valovic ${ }^{6}$, D. Van Eester ${ }^{38}$, B. van Milligen ${ }^{11}$, G.J. van Rooij ${ }^{53}$, C.A.F. Varandas ${ }^{12}$, S. Vartanian ${ }^{3}$, V. Vdovin ${ }^{16}$, J. Vega ${ }^{11}$, G. Verdoolaege ${ }^{87}$, J.M. Verger ${ }^{3}$, L. Vermare $^{3}$, C. $\operatorname{Verona}^{10}$, Th. Versloot ${ }^{53}$, M. Vervier ${ }^{38}$, J. Vicente ${ }^{12}$, S. Villari ${ }^{17}$, E. Villedieu ${ }^{3}$, F. Villone ${ }^{7}$, J.E. Vince ${ }^{6}$, G.J. Vine ${ }^{6}$, B. Viola ${ }^{7}$, E. Vitale ${ }^{17}$, R. Vitelli ${ }^{10}$, M. Vlad ${ }^{19}$, I. Voitsekhovitch ${ }^{6}$, M. Vrancken ${ }^{38}$, K. Vulliez $^{3}$, C.W.F. Waldon ${ }^{6}$, M. Walker ${ }^{6}$, M.J. Walsh ${ }^{6}$, J. Waterhouse ${ }^{6}$, M.L. Watkins ${ }^{6,24}$, M.J. Watson ${ }^{6}$, T. Wauters ${ }^{3}$, M.W. Way ${ }^{6}$, C.R. Webb ${ }^{6}$, J. Weiland ${ }^{14}$, H. Weisen ${ }^{35,24}$, M. Weiszflog ${ }^{15}$, R. Wenninger ${ }^{20}$, A.T. West ${ }^{6}$, J.M. Weulersse ${ }^{85}$, B. Weyssow ${ }^{80,39}$, M.R. Wheatley ${ }^{6}$, A.D. Whiteford ${ }^{82}$, A.M. Whitehead ${ }^{6}$, A.G. Whitehurst ${ }^{6}$, A.M. Widdowson ${ }^{6}$, R.C. Wieggers ${ }^{53}$, C. Wiegmann ${ }^{29}$, S. Wiesen ${ }^{29}$, A. Wilson ${ }^{6}$, D. Wilson ${ }^{6}$, D.J. Wilson ${ }^{6}$, H.R. Wilson ${ }^{88}$, M. Wischmeier ${ }^{20}$, D.M. Witts ${ }^{6}$, R.C. Wolf ${ }^{29}$, J. Wolowski ${ }^{34}$, P. Woscov ${ }^{74}$, G.M. Wright ${ }^{53}$, J. Wright ${ }^{74}$, G.S. $\mathrm{Xu}^{89}$, V. Yavorskij ${ }^{61}$, V. Yerashok ${ }^{37}$, J. Yorkshades ${ }^{6}$, C. Young ${ }^{6}$, D. Young 6 , I.D. Young 6 , X. Yuhong ${ }^{38}$, L. Zabeo ${ }^{6}$, A. Zabolotsky ${ }^{35}$, L. Zaccarian ${ }^{17}$, R. Zagorski ${ }^{39,34}$, F.S. Zaitsev ${ }^{76}$, S. Zajac ${ }^{34}$, L. Zakharov ${ }^{44}$, R. Zanino ${ }^{83}$, V. Zaroschi ${ }^{19}$, K.D. Zastrow ${ }^{6}$, I. Zatz ${ }^{44}$, B. Zefran ${ }^{75}$, W. Zeidner ${ }^{20}$, M. Zerbini ${ }^{17}$, T. Zhang ${ }^{29}$, Y. Zhu ${ }^{89}$, E. Zilli27, O. Zimmermann ${ }^{29}$, V. Zoita ${ }^{24,19}$, S. Zoletnik ${ }^{13}$, W. Zwingman ${ }^{3}$

${ }^{1}$ Imperial College, University of London, London, SW7 2AZ, UK

${ }^{2}$ Ioffe Physico-Technical Institute, 26 Politekhnicheskaya, St Petersburg 194021, Russian Federation

${ }^{3}$ Association EURATOM-CEA, CEA/DSM/IRFM, Cadarache 13108 Saint Paul Lez Durance, France

${ }^{4}$ Department of Pure and Applied Physics, Queens University, Belfast, BT7 1NN, UK

${ }^{5}$ VTT Technical Research Centre of Finland, Association EURATOM-Tekes, PO Box 1000, FIN-02044 VTT, Finland

${ }^{6}$ Euratom/CCFE Fusion Association, Culham Science Centre, Abingdon, Oxon, OX14 3DB, UK

${ }^{7}$ Associazione EURATOM-ENEA sulla Fusione, Consorzio CREATE, Via Claudio 21, 80125 Napoli, Italy

${ }^{8}$ Troitsk Insitute of Innovating and Thermonuclear Research (TRINITI), Troitsk 142190, Moscow Region, Russian Federation 
${ }^{9}$ Associazione EURATOM-ENEA sulla Fusione, Consorzio RFX Padova, Italy

${ }^{10}$ Associazione EURATOM-ENEA sulla Fusione, Università di Roma, Italy

${ }^{11}$ Laboratorio Nacional de Fusion, Asociacion EURATOMCIEMAT, Madrid, Spain

${ }^{12}$ Associação EURATOM/IST, Instituto de Plasmas e Fusão Nuclear, Instituto Superior Técnico, Av Rovisco Pais, 1049-001 Lisbon, Portugal

${ }^{13}$ KFKI-Research Institute for Particle and Nuclear Physics, Association EURATOM, PO Box 49, H-1525, Budapest, Hungary

${ }^{14}$ Association EURATOM-VR, Department of Earth and Space Sciences, Chalmers University of Technology, SE-41296 Gothenburg, Sweden

${ }^{15}$ Association EURATOM-VR, Department of Physics and Astronomy, Uppsala University, SE-75120 Uppsala, Sweden

${ }^{16}$ RRC Kurchatov Institute, 1 Kurchatov Square, Moscow 123182, Russian Federation

${ }^{17}$ Associazione EURATOM-ENEA sulla Fusione, C.R. Frascati, Roma, Italy

${ }^{18}$ The National Institute for Cryogenics and Isotopic Technology, Association EURATOM-MEdC, Ramnicu Valcea, Romania

19 The National Institute for Laser, Plasma and Radiation Physics, Association EURATOM-MEdC, MagureleBucharest, Romania

${ }^{20}$ Max-Planck-Institut für Plasmaphysik, EURATOMAssoziation, D-85748 Garching, Germany

${ }^{21}$ Dipartimento di Ingegneria Elettrica Elettronica e dei Sistemi-Università degli Studi di Catania, 95125 Catania, Italy

${ }^{22}$ Dublin City University (DCU), Ireland

${ }^{23}$ Fusion for Energy Joint Undertaking, Josep Pl. 2, Torres Diagonal Litoral B3, 08019, Barcelona, Spain

${ }^{24}$ EFDA Close Support Unit, Culham Science Centre, Culham, OX14 3DB, UK

${ }^{25}$ Aalto University, Association EURATOM-Tekes, PO Box 14100, FIN-00076 Aalto, Finland

26 The Nuclear Fuel Plant, Pitesti, Romania

${ }^{27}$ Associazione EURATOM-ENEA sulla Fusione, IFP Milano, Italy

${ }^{28}$ Lehigh University, Bethlehem, PA 18015, USA

${ }^{29}$ Forschungszentrum Jülich, Institute of Energy ResearchPlasma Physics, EURATOM Association, D-52425, Jülich, Germany

${ }^{30}$ Oak Ridge National Laboratory, Oak Ridge, TN 378316169, USA

${ }^{31}$ Karlsruhe Institute of Technology, PO Box 3640, D-76021 Karlsruhe, Germany

${ }^{32}$ Association EURATOM-VR, Fusion Plasma Physics, EES, KTH, SE-10044 Stockholm, Sweden

${ }^{33}$ University of Texas at Austin, Institute for Fusion Studies, Austin, TX 78712, USA

${ }^{34}$ Association Euratom-IPPLM, Hery 23, 01-497 Warsaw, Poland

${ }^{35}$ Association EURATOM-Confédération Suisse, Ecole Polytechnique Fédérale de Lausanne (EPFL), CRPP, CH-1015 Lausanne, Switzerland
${ }^{36}$ Laboratoire J.A.Dieudonné, Université de Nice-SophiaAntipolis, Parc Valrose, F-06108 Nice CEDEX 02, France

${ }^{37}$ Magnetic Sensor Laboratory (LPNU), 1 Kotliarevsky Str, Lviv, 79013, Ukraine

${ }^{38}$ Association 'EURATOM-Belgian State' Laboratory for Plasma Physics Koninklijke Militaire School-Ecole Royale Militaire Renaissancelaan 30 Avenue de la Renaissance B-1000 Brussels, Belgium

${ }^{39}$ EFDA Close Support Unit, D-85748 Garching, Germany

${ }^{40}$ Association EURATOM-SCK-CEN, Nuclear Research Centre, 2400 Mol, Belgium

${ }^{41}$ The National Institute for Optoelectronics, MagureleBucharest, Romania, Association EURATOM-MEdC

${ }^{42}$ Max-Planck-Institut für Plasmaphysik, Teilinsitut Greifswald, EURATOM-Assoziation, D-17491 Greifswald, Germany

${ }^{43}$ Forschungszentrum Jülich, Institute of Energy Research, EURATOM Association, D-52425, Jülich, Germany

${ }^{44}$ Princeton Plasma Physics Laboratory, James Forrestal Campus, Princeton, NJ 08543, USA

${ }^{45}$ General Atomics, PO Box 85608, San Diego, CA 921865608, USA

${ }^{46}$ Department of Electrical and Electronic Engineering, University of Cagliari, Piazza d'Armi 09123 Cagliari, Italy

${ }^{47}$ University of California, 1111 Franklin St., Oakland, CA 94607, USA

${ }^{48}$ Colorado School of Mines, 1500 Illinois Street, Golden, CO 80401, USA

49 Japan Atomic Energy Agency, Naka Fusion Research Establishment, Nakamachi, Naka-gun, Ibaraki-ken 311-0913, Japan

${ }^{50}$ Department of Applied Physics UG (Ghent University) Rozier 44 B-9000 Ghent, Belgium

${ }^{51}$ CEA/Fontenay aux Roses, B.P.6 F-92265 Fontenay-auxroses CEDEX, France

52 Universidad Politécnica de Madrid, Grupo I2A2, Madrid, Spain

${ }^{53}$ FOM Institute for Plasma Physics Rijnhuizen, Association EURATOM-FOM, Trilateral Euregio Cluster, The Netherlands

${ }^{54}$ Institute of Applied Physics, Nizhny Novgorod 603155, Russian Federation

${ }^{55}$ Bulgarian Academy of Sciences, 6 Moskovsak Str., Sofia 1000, Bulgaria

${ }^{56}$ Association EURATOM-IPP.CR, Institute of Plasma Physics AS CR, Za Slovankou 3, 18221 Praha 8, Czech Republic

${ }^{57}$ Association EURATOM-VR, Department of Material Physics, ICT, KTH, SE-16440 Kista, Sweden

${ }^{58}$ European Commission, B-1049 Brussels, Belgium

${ }^{59}$ Association EURATOM-Hellenic Republic, NCSR 'Demokritos' 153 10, Agia Paraskevi Attikis, Greece

${ }^{60}$ Physikalisch-Technische Bundesanstalt, Bundesallee 100, D-38116, Braunschweig, Germany

${ }^{61}$ Association EURATOM-Österreichische Akademie der Wissenschaften (ÖAW), Austria

${ }^{62}$ Institute for Plasma Research, University of Maryland, College Park, MD 20742-3511, USA

${ }^{63}$ Seoul National University, Shilim-Dong, Gwanak-Gu, Republic of Korea 
${ }^{64}$ ITER Organization, Route de Vinon, CS 90 046, 13067 Saint Paul Lez Durance, France

${ }^{65}$ Daegu University, Jillyang, Gyeongsan, Gyeongbuk 712-174, Republic of Korea

${ }^{66}$ Association EURATOM-LEI, Breslaujos str. 3, LT-44403, Kaunas, Lithuania

${ }^{67}$ Association EURATOM-VR, Department of Physics, Lund University, SE-22100 Lund, Sweden

${ }^{68}$ Association EURATOM-VR, Department of Engineering Sciences, Uppsala University, SE-75120 Uppsala, Sweden

${ }^{69}$ Physics Section, Division of Physical and Chemical Sciences, International Atomic Energy Agency, PO Box 100, Wagramer Strasse 5, A-1400 Vienna, Austria

${ }^{70}$ Association EURATOM-Hellenic Republic, National Technical University of Athens, Iroon Politechniou 9, 15773 Zografou, Athens, Greece

${ }^{71}$ IPF, Stuttgart University, Stuttgart, Germany

${ }^{72}$ University of Tartu, Ülikooli 18, 50090 Tartu, Estonia

${ }^{73}$ University of Latvia, 19 Raina Blvd., Riga, LV 1586, Latvia

${ }^{74}$ MIT Plasma Science and Fusion Centre, Cambridge, MA 02139, USA

75 Association EURATOM-MHST, Jozef Stefan Institute, Reactor Physics Department, Jamova 39, SI-1000 Ljubljana, Slovenia

${ }^{76}$ Moscow State University, Moscow 119991, Russian Federation

77 Association EURATOM-Ris $\varnothing$ National Laboratory, Technical University of Denmark, PO Box 49, DK-4000 Roskilde, Denmark

78 Departamento de Física, Universidad Carlos III de Madrid, 28911 Leganés, Madrid, Spain

${ }^{79}$ Association EURATOM-VR, Department of Physics, SCI, KTH, SE-10691 Stockholm, Sweden

${ }^{80}$ Statistical and Plasma Physics Unit, Association EURATOM-Belgian State, Université Libre de Bruxelles, Campus Plaine, CP 231, Boulevard du Triomphe, B-1050, Belgium

${ }^{81}$ The 'Horia Hulubei' National Institute for Physics and Nuclear Engineering, Association EURATOM-MEdC, Magurele-Bucharest, Romania

${ }^{82}$ Department of Physics and Applied Physics, University of Strathclyde, Glasgow, G4 ONG, UK

${ }^{83}$ Associazione EURATOM-ENEA sulla Fusione, Politecnico di Torino, Italy

${ }^{84}$ Department of Physics, University of Warwick, Coventry, CV4 7AL, UK

${ }^{85}$ CEA/Saclay, F-91191 Gif-sur-Yvette CEDEX, France

${ }^{86}$ Tampere University of Technology, Association EURATOM-Tekes, PO Box 527, FI-33101 Tampere, Finland

${ }^{87}$ Association EURATOM-Belgian State Department of Data Analysis, Ghent University, 9000 Gent, Belgium

${ }^{88}$ University of York, Heslington, York YO10 5DD, UK

${ }^{89}$ Institute of Plasma Physics, Chinese Academy of Sciences, Hefei, 230031, People's Republic of China

\section{References}

[1] Romanelli F. et al 2008 Proc. 22nd Int. Conf. on Fusion Energy 2008 (Geneva, Switzerland, 2008) (Vienna: IAEA) paper OV/1-2 and http://www-naweb.iaea.org/napc/physics/ FEC/FEC2008/html/index.htm

[2] ITER Physics Basis Editors et al 1999 Chapter 1: Overview and summary Nucl. Fusion 39 2137-74

[3] Paméla J. et al 2007 J. Nucl. Mater. 363-365 1-11

[4] Philipps V. et al 2010 Fusion Eng. Des. 85 1581-6

[5] Nunes I. et al 2010 Proc. 23rd Int. Conf. on Fusion Energy 2010 (Daejeon, Korea, 2010) (Vienna: IAEA) paper EXC/P8-03 and http://www-naweb.iaea.org/napc/ physics/FEC/FEC2010/html/index.htm

[6] Ćirić D. et al 2007 Fusion Eng. Des. 82 610-8

[7] Ruset C. et al 2007 Phys. Scr. T128 171-4

[8] Ruset C. et al 2010 Development of W coatings for fusion applications Fusion Eng. Des. at press (doi:10.1016/j.fusengdes.2011.04.031)

[9] Mertens Ph. et al 2009 Fusion Eng. Des. 84 1289-93

[10] Mertens Ph. et al 2011 A bulk tungsten tile for JET: derivation of power-handling performance and validation of the thermal model, in the MARION Facility Fusion Eng. Des. at press (doi:10.1016/j.fusengdes.2011.03.044)

[11] Ganuza D. et al 2009 Fusion Eng. Des. 84 810-4

[12] Shaw S.R. et al 2010 The installation, testing and performance on the JET coils on the enhanced radial field amplifier (ERFA) Fusion Eng. Des. submitted

[13] Rimini F.G. et al 2011 First plasma operation of the enhanced JET vertical stabilisation system Fusion Eng. Des. at press (doi:10.1016/j.fusengdes.2011.03.122)

[14] Huber A. et al 2010 Radiation loads onto plasma-facing components of JET during transient events-experimental results and implications for ITER $J$. Nucl. Mater. at press (doi:10.1016/j.jnucmat.2010.10.061)

[15] ITER Physics Expert Group on Confinement and Transport, ITER Physics Expert Group on Confinement Modelling and Database and ITER Physics Basis Editors 1999 Chapter 2: Plasma confinement and transport Nucl. Fusion 39 2175-249

[16] Beurskens M.N.A. et al 2011 Phys. Plasmas 18056120

[17] Osborne T.H. et al 2010 Proc. 23rd Int. Conf. on Fusion Energy 2010 (Daejeon, Korea, 2010) (Vienna: IAEA) paper EXC/2-1 and http://www-naweb.iaea.org/napc/physics/ FEC/FEC2010/html/index.htm

[18] Sartori R. et al 2010 Proc. 23rd Int. Conf. on Fusion Energy 2010 (Daejeon, Korea, 2010) (Vienna: IAEA) paper EXC/P8-12 and http://www-naweb.iaea.org/napc/physics/ FEC/FEC2010/html/index.htm

[19] Martin Y. et al 2008 J. Phys. Conf. Ser 123012033

[20] Joffrin E. et al 2009 Proc. 22nd Int. Conf. on Fusion Energy 2008 (Geneva, Switzerland, 2009) (Vienna: IAEA) paper EX/1-4Ra and http://www-naweb.iaea.org/napc/physics/ FEC/FEC2008/html/index.htm

[21] Joffrin E. et al 2010 Proc. 23rd Int. Conf. on Fusion Energy 2010 (Daejeon, Korea, 2010) (Vienna: IAEA) paper EX/1-1 and http://www-naweb.iaea.org/napc/physics/ FEC/FEC2010/html/index.htm

[22] McDonald D.C. et al 2008 Plasma Phys. Control. Fusion 50124013

[23] Politzer P. et al 2010 Proc. 23rd Int. Conf. on Fusion Energy 2010 (Daejeon, Korea, 2010) (Vienna: IAEA) paper EXC/P2-06 and http://www-naweb.iaea.org/napc/physics/ FEC/FEC2010/html/index.htm

[24] Goldston R.J. et al 1981 J. Comput. Phys. 4361

[25] Artaud J.F. et al 2010 Nucl. Fusion 50043001

[26] Mantica P. et al 2010 Proc. 23rd Int. Conf. on Fusion Energy 2010 (Daejeon, Korea, 2010) (Vienna: IAEA) paper EXC/9-2 and http://www-naweb.iaea.org/napc/physics/ FEC/FEC2010/html/index.htm

[27] Frassinetti L. et al 2010 Proc. 37th EPS Conf. on Plasma Physics (Dublin, Ireland, 2010) vol 34A (ECA) (Geneva: European Physical Society) paper P1.1031 http://ocs.ciemat.es/EPS2010PAP/pdf/P1.1031.pdf

[28] Mailloux J. et al 2010 Proc. 23rd Int. Conf. on Fusion Energy 2010 (Daejeon, Korea, 2010) (Vienna: IAEA) paper 
EXC/1-4 and http://www-naweb.iaea.org/napc/physics/ FEC/FEC2010/html/index.htm

[29] Garcia J. et al 2009 Radio frequency power in plasmas AIP Conf. Proc. 1187 31-8

[30] Garcia J. et al 2010 Impact of off-axis RF current drive on JET advanced scenarios Nucl. Fusion submitted

[31] Giruzzi G. et al 2010 Proc. 23rd Int. Conf. on Fusion Energy 2010 (Daejeon, Korea, 2010) (Vienna: IAEA) paper EXW/P7-04 and http://www-naweb.iaea.org/napc/physics/ FEC/FEC2010/html/index.htm

[32] Lackner K. et al 1994 Plasma Phys. Control. Fusion 36 B79

[33] Righi E. 1999 Nucl. Fusion 39309

[34] Sips A.C.C. et al 2009 Nucl. Fusion 49085015

[35] Sips A.C.C. et al 2010 Proc. 23rd Int. Conf. on Fusion Energy 2010 (Daejeon, Korea, 2010) (Vienna: IAEA) paper EXC/P2-08 and http://www-naweb.iaea.org/napc/physics/ FEC/FEC2010/html/index.htm

[36] McDonald D.C. et al 2010 Proc. 23rd Int. Conf. on Fusion Energy 2010 (Daejeon, Korea, 2010) (Vienna: IAEA) paper EXC/2-4Rb and http://www-naweb.iaea.org/napc/physics/ FEC/FEC2010/html/index.htm

[37] Ryter F. et al 2009 Nucl. Fusion 49062003

[38] Gohil P. et al 2010 Nucl. Fusion 50064011

[39] McDonald D.C. et al 2004 Plasma Phys. Control. Fusion 46519

[40] Fundamenski W. et al 2010 Proc. 23rd Int. Conf. on Fusion Energy 2010 (Daejeon, Korea, 2010) (Vienna: IAEA) paper EXD/P3-11 and http://www-naweb.iaea.org/napc/ physics/FEC/FEC2010/html/index.htm

[41] Tala T. et al 2010 Proc. 23rd Int. Conf. on Fusion Energy 2010 (Daejeon, Korea, 2010) (Vienna: IAEA) paper EXC/3-1 and http://www-naweb.iaea.org/napc/physics/ FEC/FEC2010/html/index.htm

[42] Peeters A.G. and Strintzi D. 2004 Phys. Plasmas 113748

[43] Kotschenreuther M. et al 1995 Comput. Phys. Commun. 88128

[44] Nave M.F.F. et al 2010 Phys. Rev. Lett. 105105005

[45] Rice J.E. et al 2007 Nucl. Fusion 471618

[46] Kiptily V.G. et al 2009 Nucl. Fusion 49065030

[47] Kiptily V.G. et al 2010 Proc. 23rd Int. Conf. on Fusion Energy 2010 (Daejeon, Korea, 2010) (Vienna: IAEA) paper EXS/P7-01 and http://www-naweb.iaea.org/napc/ physics/FEC/FEC2010/html/index.htm

[48] Gorelenkov N.N. et al 2003 Phys. Plasmas 10713

[49] Perez von Thun C. et al 2010 Nucl. Fusion 50084009

[50] Perez von Thun C. et al 2011 Nucl. Fusion 51053003

[51] Chen L. et al 1984 Phys. Rev. Lett. 521122

[52] Coppi B. et al 1988 Fusion Technol. 13447

[53] Mikhailovskii A.B. et al 1997 Plasma Phys. Rep. 23844

[54] Pinches S.D. et al 1998 Comput. Phys. Commun. 111133

[55] Hedin J. et al 2002 Nucl. Fusion 42527

[56] Graves J.P. et al 2010 Proc. 23rd Int. Conf. on Fusion Energy 2010 (Daejeon, Korea, 2010) (Vienna: IAEA) paper THS/9-1 and http://www-naweb.iaea.org/napc/physics/ FEC/FEC2010/html/index.htm

[57] Graves J.P. et al 2009 Phys. Rev. Lett. 102065005

[58] Laxåback M. et al 2005 Nucl. Fusion 451510

[59] Matthews G. et al 2009 Phys. Scr. T138 014030

[60] Brezinsek S. et al 2010 Overview of experimental preparation for the ITER-like wall at JET J. Nucl. Mater. at press (doi:10.1016/j.jnucmat.2010.10.037)

[61] de la Luna E. et al 2010 Proc. 23rd Int. Conf. on Fusion Energy 2010 (Daejeon, Korea, 2010) (Vienna: IAEA) paper EXC/8-4 and http://www-naweb.iaea.org/napc/physics/ FEC/FEC2010/html/index.htm

[62] Liang Y. et al 2010 Proc. 23rd Int. Conf. on Fusion Energy 2010 (Daejeon, Korea, 2010) (Vienna: IAEA) paper EXS/P3-04 and http://www-naweb.iaea.org/napc/ physics/FEC/FEC2010/html/index.htm
[63] Lang P.T. et al 2011 Nucl. Fusion 51033010

[64] Evans T.E. et al 2005 Nucl. Fusion 45595

[65] Evans T.E. et al 2006 Nature Phys. 2419

[66] Liang Y. et al 2010 Nucl. Fusion 50025013

[67] Liang Y. et al 2010 Phys. Rev. Lett. 105065001

[68] Gimblett C.G. et al 2006 Phys. Rev. Lett. 96035006

[69] Köchl F. et al 2010 Proc. 37th EPS Conf. on Plasma Physics (Dublin, Ireland, 2010) vol 34A (ECA) (Geneva: European Physical Society) paper O4.123 http://ocs.ciemat.es/EPS2010PAP/pdf/O4.123.pdf

[70] Eich T. et al 2011 Type-I ELM power deposition profile width and temporal shape in JET J. Nucl. Mater. at press (doi:10.1016/j.jnucmat.2010.11.079)

[71] Thomsen H. et al 2010 Proc. 23rd Int. Conf. on Fusion Energy 2010 (Daejeon, Korea, 2010) (Vienna: IAEA) paper EXD/6-6Rb and http://www-naweb.iaea.org/napc/ physics/FEC/FEC2010/html/index.htm

[72] Devaux S. et al 2011 Type-I ELM filamentary substructure on the JET divertor target $J$. Nucl. Mater. at press (doi:10.1016/j.jnucmat.2011.01.050)

[73] Hender T.C. et al 2010 Proc. 23rd Int. Conf. on Fusion Energy 2010 (Daejeon, Korea, 2010) (Vienna: IAEA) paper EXS/10-3 and http://www-naweb.iaea.org/napc/physics/ FEC/FEC2010/html/index.htm

[74] Lehnen M. et al 2010 Proc. 23rd Int. Conf. on Fusion Energy 2010 (Daejeon, Korea, 2010) (Vienna: IAEA) paper EXS/P2-13 and http://www-naweb.iaea.org/napc/physics/ FEC/FEC2010/html/index.htm

[75] de Vries P.C. et al 2011 Nucl. Fusion 51053018

[76] Riccardo V. et al 2010 Plasma Phys. Control. Fusion 52124018

[77] ITER Physics Expert Group on Disruptions, Plasma Control, and MHD and ITER Physics Basis Editors 1999 Chapter 3: MHD stability, operational limits and disruptions $\mathrm{Nucl}$. Fusion 39 2251-389

[78] Gerasimov S. et al 2010 Proc. 37th EPS Conf. on Plasma Physics (Dublin, Ireland, 2010) vol 34A (ECA) (Geneva European Physical Society) paper P4.121

[79] Arnoux G. et al 2010 Heat load measurements on the JET first wall during disruptions $\mathrm{J}$. Nucl. Mater. at press (doi:10.1016/j.jnucmat.2010.11.042)

[80] Dreicer H. 1959 Phys. Rev. 115 238-49

[81] Progress in the ITER Physics Basis 2007 Chapter 3: MHD stability, operational limits and disruptions Nucl. Fusion 47 S128-S202

[82] Durodié F. et al 2010 Proc. 23rd Int. Conf. on Fusion Energy 2010 (Daejeon, Korea, 2010) (Vienna: IAEA) paper EXW/P7-04 and http://www-naweb.iaea.org/napc/ physics/FEC/FEC2010/html/index.htm

[83] Durodié F. et al 2001 Radio frequency power in plasmas AIP Conf. Proc. $\mathbf{5 9 5} 122$

[84] Durodié F. et al 2005 Fusion Eng. Des. 74 223-8

[85] Durodié F. et al 2009 Radio frequency power in plasmas AIP Conf. Proc. 1187 221-4

[86] Nightingale M.P.S. et al 2009 Radio frequency power in plasmas AIP Conf. Proc. 1187 213-20

[87] Bora D. et al 2007 Radio frequency power in plasmas AIP Conf. Proc. 933 25-32

[88] Vrancken M. et al 2011 Performance of the scattering matrix arc detection system on the JET ITER-like ICRF antenna Fusion Eng. Des. at press (doi:10.1016/j.fusengdes.2011.02.056)

[89] Jacquet P., et al 2009 Radio frequency power in plasmas AIP Conf. Proc. 1187 241-4

[90] Lancellotti V. et al 2006 Nucl. Fusion 46 S476

[91] Milanesio D. et al 2007 Plasma Phys. Control. Fusion 49405

[92] Maggiora R. et al 2004 Nucl. Fusion 44846 